\title{
Matching, voting and cooperation
}

Citation for published version (APA):

Kasper, L. (2019). Matching, voting and cooperation. [Doctoral Thesis, Maastricht University]. ProefschriftMaken Maastricht. https://doi.org/10.26481/dis.20190221/k

Document status and date:

Published: 01/01/2019

DOI:

10.26481/dis.20190221lk

Document Version:

Publisher's PDF, also known as Version of record

\section{Please check the document version of this publication:}

- A submitted manuscript is the version of the article upon submission and before peer-review. There can be important differences between the submitted version and the official published version of record.

People interested in the research are advised to contact the author for the final version of the publication, or visit the DOI to the publisher's website.

- The final author version and the galley proof are versions of the publication after peer review.

- The final published version features the final layout of the paper including the volume, issue and page numbers.

Link to publication

\footnotetext{
General rights rights.

- You may freely distribute the URL identifying the publication in the public portal. please follow below link for the End User Agreement:

www.umlib.nl/taverne-license

Take down policy

If you believe that this document breaches copyright please contact us at:

repository@maastrichtuniversity.nl

providing details and we will investigate your claim.
}

Copyright and moral rights for the publications made accessible in the public portal are retained by the authors and/or other copyright owners and it is a condition of accessing publications that users recognise and abide by the legal requirements associated with these

- Users may download and print one copy of any publication from the public portal for the purpose of private study or research.

- You may not further distribute the material or use it for any profit-making activity or commercial gain

If the publication is distributed under the terms of Article $25 \mathrm{fa}$ of the Dutch Copyright Act, indicated by the "Taverne" license above, 


\section{MATCHING, Voting AND COOPERATION}

LAURA KASPER 
(c) Laura Kasper, Maastricht 2019

All rights reserved. No part of this publication may be reproduced, stored in a retrieval system or transmitted, in any form or by any means, electronic, mechanical, photocopying, recording or otherwise, without prior permission of the author or the copyright-owning journals for previous published chapters.

This book was typeset by the author using $\mathrm{IAT}_{\mathrm{E}} \mathrm{X}$ and the classicthesis package.

Printing: ProefschriftMaken II www.proefschriftmaken.nl

ISBN 9789463802451 


\title{
MATCHING, Voting AND COOPERATION
}

\section{DISSERTATION}

\author{
to obtain the degree of Doctor \\ at Maastricht University,
}

on the authority of the Rector Magnificus

Prof. dr. Rianne M. Letschert,

in accordance with the decision of the Board of Deans,

to be defended in public

on Thursday 21st of February 2019, at 16.00 hours

by

LAURA KASPER 


\section{Supervisor}

Prof. dr. Hans Peters

Prof. dr. Dinko Dimitrov

Prof. dr. Dries Vermeulen

\section{Co-supervisor}

Dr. Dominik Karos

\section{Assessment Committee}

Prof. dr. Rudolf Müller (chair)

Prof. dr. Tommy Andersson (Lund University, Sweden)

Prof. dr. Michel Grabisch (Université Paris 1 Panthéon-Sorbonne, France)

Prof. dr. Antonius Storcken 
To my family.

Für meine Familie. 

This thesis represents a summary of my research efforts over the last three years. There are many people who deserve my thankfulness. I will thank some people in particular. First of all, I would like to thank my supervisors Hans Peters, Dinko Dimitrov and Dries Vermeulen. I appreciate the time and effort that all of you invested in joint research, and, in particular, in helping me to become a researcher. I am very grateful for the patience you had with me and for the support you provided. I would like to thank Dinko Dimitrov for giving me the chance to work with him at Saarland University and for the financial support, which has allowed me to visit many conferences and summer schools over the last three years. I would like to thank Hans Peters and Dries Vermeulen for giving me the opportunity to join Maastricht University and for fruitful discussions about research. Thank you for your advise and understanding. I also would like to thank my co-supervisor and friend Dominik Karos. Thank you for your support. Moreover, I thank the members of my thesis committee Tommy Andersson, Michel Grabisch, Rudolf Müller and Ton Storcken. Thank you for reading and approving this thesis. I also thank my paranimphs, Toygar Kerman and Francisco Robles.

During the last three years, I have had the great chance to do some research stays, and I would like to thank Tommy Andersson and Agniezska Rusinowska for inviting me. Tommy Andersson, thank you for our weekly discussions in Lund. They helped me grow as a researcher and as a person. I also thank all participants of the microeconomic theory seminar at Lund University for their helpful comments. In particular, I would like to thank Jens Gudmundsson and Alex Rigos for many discussions about research and private life. Thank you to the other PhD students in Lund for discussions about research, board game nights and other social activities. Thank you Agnieszka Rusinowska for giving me the opportunity to present my work at Université Paris 1 Panthéon-Sorbonne in Paris. I really appreciate your support. Thank you, Xavier Venel, for the fruitful discussion about my paper. I also thank all participants of the CES Economic theory seminar in Paris.

I thank the PhD students at Maastricht University and the participants of the MSLE seminar for valuable comments on my research. Thank you Claudia Philipps and Patrick Keßler for your work and support at Saarland University. Thank you Yongjie Yang for being my coauthor. I also would like to thank Ata Atay for the great time we spent together at conferences. You became a friend to me.

Last, but not least, I would like to express my gratitude towards some people of my private life. Thank you, Kristina Backes, for being my longest and dearest friend. Thank 
you for always having an open ear and supporting me. Thank you Karolina Kowol, Christian Agethen and Lisa Grotefend for being such good friends. I always have a great time with you. Thank you, Francisco Robles, for always helping and supporting me. Thank you for being the most funny and caring person I know. You have my love. I would like to thank my family. Thank you for your eternal love. Thank you, to my mother and father, there is no way that this would be happening without you. I am deeply grateful for everything you have done. 


\section{CONTENTS}

1 INTRODUCTION 1

2 EMPLOYMENT EXPERIENCE AND STABILITY 7

2.1 Introduction ....................... 7

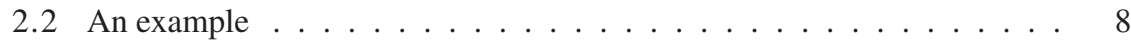

2.3 Experience dependent problems ................... 9

2.4 Experience size-sensitivity . . . . . . . . . . . . . . . 9

2.5 Stable matchings . . . . . . . . . . . . . . . . 10

2.6 Non-existence of firm optimal stable matchings . . . . . . . . . . 13

3 GENDER CONSISTENT RESOLVING RULES IN MARRIAGE PROBLEMS 15

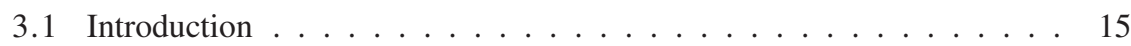

3.2 Setup and axioms . . . . . . . . . . . . . . . . 16

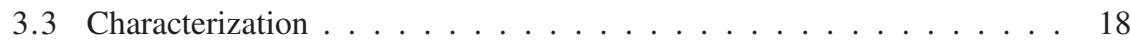

4 CONDORCET CONSISTENCY AND THE STRONG NO SHOW PARADOXES 21

4.1 Introduction . . . . . . . . . . . . . . . 21

4.2 Basic definitions . . . . . . . . . . . . . . . 23

$4.3 \mathrm{CC}, \mathrm{TP}$ and $\mathrm{BP}$ for voting correspondences . . . . . . . . . . . . 24

$4.4 \mathrm{CC}, \mathrm{TP}$ and $\mathrm{BP}$ for voting functions $\ldots \ldots \ldots . \ldots 30$

4.4.1 Selecting independently . . . . . . . . . . . . . . 31

4.4 .2 Non-independent selections . . . . . . . . . . . . . . . 34

4.5 Weak Condorcet Consistency . . . . . . . . . . . . . . . . 35

4.6 Concluding remarks . . . . . . . . . . . . . . . 36

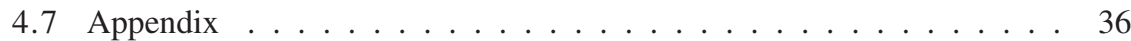

5 FULL FARSIGHTED RATIONALITY 43

5.1 Introduction . . . . . . . . . . . . . . . . . . 43

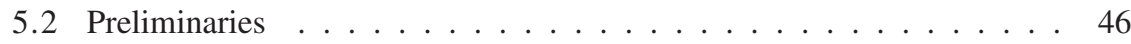

5.2 .1 Notation . . . . . . . . . . . . . . . . . . 46

5.2 .2 Expectation functions . . . . . . . . . . . . . 47

5.3 Rational expectations . . . . . . . . . . . . . . . . 47

5.3.1 Rational expectation functions . . . . . . . . . . . . . 47

5.3 .2 Extended expectation functions . . . . . . . . . . . . 50

5.4 Examples ............................. 52

5.5 Non-cooperative foundation . . . . . . . . . . . . . . . . 55

5.5.1 Strategies and extended expectation functions . . . . . . . 55 
5.5 .2 Better responses . . . . . . . . . . . . . . 56

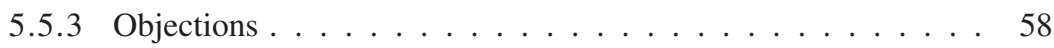

5.5 .4 Incredible threats . . . . . . . . . . . . . . . . . . . . . . . . . . . . . . . 69

5.5 .5 Best responses ..................... 61

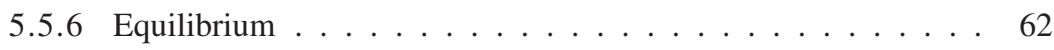

5.6 Applications . . . . . . . . . . . . . . . 66

5.6.1 Absorbing extended expectation functions . . . . . . . . . . 66

5.6.2 Single-Payoff Equilibrium Stable Sets . . . . . . . . . . . 67

5.6 .3 Characteristic function form games . . . . . . . . . . 67

5.6 .4 Matching . . . . . . . . . . . . . . . 68

5.7 Conclusion . . . . . . . . . . . . . . . . . 69

6 INTERTEMPORAL TRANSFERS IN DYNAMIC TU-GAMES 71

6.1 Introduction . . . . . . . . . . . . . 71

6.2 Preliminaries . . . . . . . . . . . . . . . . 74

6.2 .1 Transferable utility games . . . . . . . . . . . . . . 74

6.2 .2 Intertemporal utility functions . . . . . . . . . . . 78

6.2.3 Games played in a constrained environment . . . . . . . . . 79

6.3 Individual transfers and Nash equilibrium . . . . . . . . . . . 80

6.3 .1 Strategic interaction . . . . . . . . . . . . . . 80

6.3 .2 Feasibility constraints . . . . . . . . . . . . 83

6.3 .3 Existence of Nash equilibrium . . . . . . . . . . . . 86

6.3.4 Characterization of Nash equilibrium . . . . . . . . . . . . . . . . . . . . .

6.3.5 Uniqueness of Nash equilibrium . . . . . . . . . . . . . . . . 94

6.4 Transfer of initial endowments . . . . . . . . . . . . . . . . . . . . . . 96

6.4 .1 Strategic interaction . . . . . . . . . . . . . . . 97

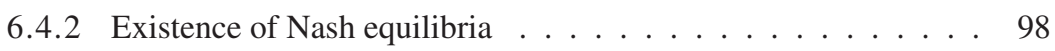

6.4 .3 Uniqueness of Nash equilibria . . . . . . . . . . . . . 101

6.5 Transfer of power . . . . . . . . . . . . . . . . . 102

6.6 Further research . . . . . . . . . . . . . . . . . . . . 106

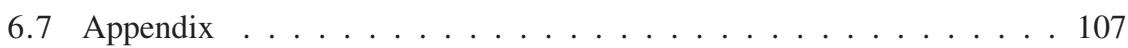

$\begin{array}{ll}\text { BIBLIOGRAPHY } & 111\end{array}$

$\begin{array}{ll}\text { SUMMARY } & 119\end{array}$

VALORISATION

$\begin{array}{ll}\text { CURRICUlum VitaE } & 125\end{array}$ 


\section{N T R O D U C T I O N}

In our daily life, we are often confronted with problems of matching, voting and cooperation: students have to be matched to schools, and organ transplants need to be matched to patients; presidents are elected, and laws are voted on; and at work projects are undertaken in groups. This thesis aims to contribute to the research in these fields. This introduction clarifies the research areas that are considered and motivates the corresponding research questions. Additionally, we discuss the main contributions of each chapter.

\section{MATCHING}

The seminal paper of Gale \& Shapley in 1962 is the corner stone of matching theory. The model introduced in Gale \& Shapley (1962) is rather simple. A finite number of men and women have strict preferences over the corresponding opposite sex and the possibility of being single. Each men seeks to be matched to at most one woman and each woman to at most one man. The aim is to match men and women in such a way that the resulting matching is stable. A matching is stable if there is no blocking pair, and it is individually rational. The idea behind such a notion of stability is natural: avoiding market failure and unraveling. As shown by Gale \& Shapley a stable matching always exists, and it can always be computed by a deferred acceptance algorithm. Gale \& Shapley's seminal contribution was related to real-life applications shortly after. As shown by Roth (1984), Gale \& Shapley's deferred acceptance algorithm is equivalent to the algorithm used to match medical residents to hospitals since 1950s by the National Resident Matching Program. Many of the recent developments in matching theory have been driven by the wish to solve problems with features that are not present in the standard model, and yet important in practice. ${ }^{1}$

Assuming that agents' preferences depend only on the identity of the agents of the other side of the market is quite restrictive. For instance, a couple might consider possible jobs as complements and hence, additional issues arise, which finally might lead to market failure. ${ }^{2}$ Moreover, externalities might influence agents' preferences. ${ }^{3}$ Sasaki \& Toda (1996) assume that individuals' preferences depend on the realized matching in

\footnotetext{
${ }^{1}$ For surveys about matching theory we refer the reader to Roth \& Sotomayor (1990), Abdulkadiroğlu \& Sönmez (2013), and Kojima (2015).

${ }^{2}$ We refer the reader to Klaus \& Klijn (2005) who identify a preference domain for couples to guarantee stability.

${ }^{3}$ A survey on matching with externalities was recently conducted by Bando et al. (2016).
} 
the society as an expression of the fact that people do usually care also about the partners of the others.

We take a similar approach in Chapter 2 of this thesis by assuming that firms' preferences are crucially shaped by workers' employment experience. We assume that each firm has only one position to offer and that each worker can fill at most one position. Experience assigns to each worker a set of firms by which she has been employed. In this context, we first show that there are experience configurations for which no stable matching exists, and then present a sufficient condition on firms' preferences guaranteeing existence of a stable matching for any experience configuration. The condition stipulates the idea that firms are size-sensitive with respect to the experience of each worker and it also allows us to fully describe the set of stable matchings. It is well known that in the standard two-sided matching market, where agents' preferences are strict, there is always a unique stable matching that is optimal for one side of the market, i.e. all agents on one side of the market weakly prefer this stable matching to any other stable matching. However, if the model is extended in the above way, a firm optimal stable matching need not exist.

Another approach complementing the possible generalizations of the standard model focusses on axiomatic characterizations of matching mechanisms. For instance, axiomatic characterizations of the deferred acceptance algorithm and its variants are provided by Kojima \& Manea (2010), Morrill (2013), and Kojima \& Ünver (2014). In Chapter 3 we also follow an axiomatic approach, however, we focus on so-called resolving rules. Such rules guide the selection of blocking pairs when converting an arbitrary matching into a stable one and, hence, play an important role in the analysis of matching mechanisms. Abeledo \& Rothblum (1995) show that stable matchings can be reached when one starts from an arbitrary individually rational matching and iteratively selects pairs according to a gender consistent resolving rule. A resolving rule is gender consistent if it selects blocking pairs following the preferences of all men or all women. Moreover, Abeledo \& Rothblum show that gender consistent resolving rules are common for, for instance, the Roth and Vande Vate algorithm (cf. Roth \& Vande Vate, 1990) and the single proposal variant of the deferred acceptance algorithms (cf. McVitie \& Wilson, 1970). The axiomatic characterization presented in this chapter is with respect to a whole class of rules. We show that two axioms, namely independence and top optimality, transform a resolving rule into a gender consistent one. The independence axiom captures the idea that, when comparing two different pairs of a preference profile and a matching, the outcome of a resolving rule remains the same if the corresponding sets of blocking pairs are related by set inclusion. Strictly speaking, the axiom allows us to conclude that it is the set of blocking pairs that matters when a rule satisfying the axiom selects a particular pair. As for our second requirement, we slightly modify the notion of domination among blocking pairs introduced by Klijn \& Massó (2003), and require in 
the top man optimality condition any resolving rule outcome to be undominated.

\section{Voting}

A voting procedure describes how agents' preferences are aggregated in order to make a collective decision. The voters can be, for instance, committee members, legislators, registered voters, and so on. We assume that voters have preferences over alternatives or candidates. In order to fully describe a voting procedure two characteristics must be clarified: (a) the type of vote that can be submitted and (b) the way in which votes are aggregated. Possible types of votes range from publicly announcing one's favorite alternative to secretly submitting a best-to-worst ranking. The collection of votes submitted by all voters is called a profile of votes. A rule prescribes exactly how to aggregate the submitted votes. One has to carefully distinguish between voting correspondences, which assign to each profile of votes a nonempty subset of alternatives and voting functions, which assigns to each profile of votes a single alternative. One might think that within a democratic voting system it should be in the own interest of voters not to abstain from voting, but there are several papers discussing the fact that sometimes abstaining can be beneficial (cf. Fishburn \& Brams, 1983; Moulin, 1988). That abstaining may be beneficial for voters is called the no show paradox. In Chapter 4 we are particularly interested in two strong versions of the no show paradox, also discussed for instance by Felsenthal \& Nurmi (2016), and say a correspondence is immune against these two paradoxes if it satisfies the Top and Bottom Property.

Another natural and desirable property of a voting system is Condorcet Consistency (de Condorcet, 1785): if there is an alternative that is ranked above any other alternative by some (weak) majority of the voters then that alternative should be chosen. We consider voting correspondences that satisfy Condorcet Consistency, and also the Top and Bottom Property. The literature so far has only presented negative results in this context. This motivates our work and, to the best of our knowledge, we are the first to provide positive and fairly complete results. We identify the maximal voting correspondence satisfying Condorcet Consistency and the Top Property. As it turns out, this voting correspondence also satisfies the Bottom property. It contains the Minimax Rule (cf. Simpson, 1969) but it is strictly larger. The Minimax Rule selects the alternative(s) against which the maximal net number of voters is minimal. Further, as voting functions that are Condorcet Consistent and immune against the two paradoxes must select from this maximal correspondence, we demonstrate several ways in which this can or cannot be done. Moreover, we consider a weaker version of Condorcet Consistency to complete our results.

\section{COOPERATION}

According to Myerson (1991) cooperation means 'players interacting with a common purpose'. There have been taken several approaches to model cooperation among players, 
mostly with tools of cooperative game theory. A main assumption in cooperative game theory is that agents can make binding agreements. One distinguishes between games with transferable utility (TU-games) and games with non-transferable utility (NTU-games). In this thesis, we focus on TU-games. In such a game each coalition is characterized by its worth, i.e., a real number representing the payoff or utility that the coalition can achieve if it forms. Moreover, it is assumed that this payoff can be freely distributed among the members of the coalition. A solution concept for TU-games assigns to each game a (set of) payoff vector(s). Arguably, the most prominent solution concepts for TU-games are the core (cf. Gillies, 1959) and the Shapley value (cf. Shapley, 1953a).

Our contribution to the literature on cooperation is two-fold: while Chapter 5 merely focuses on the behavior of coalitions, Chapter 6 is dedicated to the analysis of individuals' behavior in situations of repeated cooperation. Chapter 5 analyses games that encompass cooperative games, in particular TU-games. Our analysis in Chapter 6 is, however, explicitly based on TU-games.

Abstract games are of the main interest in Chapter 5. An abstract game consists of a set of states and specifies which coalitions are allowed to replace one state by another one. Abstract games cover, for instance, non-cooperative games, cooperative games, and also matching problems. We particularly focus on farsightedness of the players. Agents are called farsighted if they compare the status quo to the long term outcome following their deviation rather than just to the state they deviate to. Farsightedness of the agents has been discussed in a variety of settings, such as matching problems (Mauleon et al., 2011), network formation (Mauleon \& Vannetelbosch, 2004) and hedonic games (Diamantoudi \& Xue, 2003), just to name a few. What the literature has ignored so far is that if a coalition does not move out of the status quo, it might still expect another coalition to do so. Specifically, the above definition of farsightedness implies that agents ignore this possibility in their reasoning. So, in fact, agents are not fully farsighted. This motivates the first part of the chapter. In the above literature farsightedness is modeled through some (indirect) dominance relation, i.e. one state farsightedly dominates another one, if there is a sequence of states and coalitions such that for each deviating coalition the final outcome is strictly better than the corresponding status quo. A different approach was taken by Dutta \& Vohra (2017), who model farsightedness via expectation functions. Expectation functions assign to each state a (potentially) different state and a coalition that moves from the former to the latter, thereby creating paths between states. This endows agents with an expectation about what any potential deviation entails-namely the path of the prescribed further moves. We extend these functions by capturing coalitions' expectations about the consequences of not moving out of a state. If such a function is commonly known, then coalitions know exactly what will happen after any potential move, and a dominance relation can be based on this expectation. Of course there should 
be some coherence between the expectation function itself and the dominance relation it creates, in the sense that moves should only be expected if they are beneficial. We impose three stability and optimality axioms on extended expectation functions that reflect full farsightedness and rationality.

Most criticism about dominance relations, including ours, is based on implied irrational behavior of coalitions or players. Therefore it seems natural to support our dominance relation in a non-cooperative fashion. This motivates the second part of the chapter, where we endow coalitions with rather simple strategies. A coalition's strategy specifies for any state of the abstract game whether it will remain there or deviate to another state to which it can deviate. We then show that an expectation function satisfies our axioms if and only if it can be associated with a non-cooperative equilibrium of the abstract game. We finally apply our solution to games in characteristic function form and matching problems.

Chapter 6 analyzes individuals' behavior if agents have to repeatedly cooperate with others, and it extends Berden (2007). Although cooperative game theory normally abstracts away of any strategic interaction between players, we argue that in a repeated set up players would act strategically if they could. It seems natural, that, even when conducting projects in groups, individuals try to maximize their own benefit, such as money or power. Also other factors might influence agents' behavior in groups. How should a researcher behave if, due to time constraints, she cannot work at all the projects she agreed on at the same time? We intend to incorporate and predict such (strategic) behavior by modeling non-cooperative games based on a dynamic game with transferable utility and point-wise solution concepts for them. For dynamic TU-games there is not yet a unified definition and, hence, several approaches have been pursued to model repeated cooperation of agents. Rosenthal (1990a,b) assume that the population changes over time, while Filar \& Petrosjan (2000) and Lehrer \& Scarsini (2013) allow for changes in the characteristic function. We assume that the player set is fixed, a sequence of TU-games is externally given and apply point-wise solution concepts to each TU-game separately. Based on this, we model the strategic interaction between agents by allowing them to transfer some worth among periods, and, hence, indirectly affect their payoffs and also the payoffs of the other players. We discuss different transfer systems, specifying what worth agents can transfer and identify Nash equilibria in the resulting non-cooperative games. Furthermore, we characterize the Nash equilibria and discuss uniqueness of them. We also consider particular classes of TU-games, such as market games and voting games and introduce transfer systems that seem to be suited best for these games: in market games agents can transfer their initial endowment among periods, and in the context of voting games, we allow agents to shift their voting weights over time. Based on this idea we derive non-cooperative games and further discuss existence and uniqueness of Nash equilibria. 



\section{EMPLOYMENT EXPERIENCE AND STABILITY IN TW O-SIDED MATCHING}

\subsection{INTRODUCTION}

In this chapter we reconsider the classical two-sided matching framework introduced by Gale \& Shapley (1962). ${ }^{1}$ We develop a model in which firms' preferences over workers depend on their employment experience. More precisely, we interpret the experience of a worker as the set of firms by which the worker has been employed. We show in Section 2.2 that there are experience configurations for which no stable matching exists and provide necessary notation in Section 2.3. We further present a sufficient condition on firms' preferences guaranteeing existence of a stable matching for any configuration of experience in Section 2.4. The condition stipulates the idea that firms are size-sensitive with respect to the experience of each worker. Moreover, we fully describe the set of stable matchings (see Section 2.5). We also provide an example showing that size-sensitivity with respect to experience does not assure the existence of a firm optimal stable matching (see Section 2.6).

Our model is broadly related to problems of two-sided matching with externalities, starting with the influential paper of Sasaki \& Toda (1996) and recently surveyed by Bando et al. (2016). Under the influence of externalities, individuals' preferences are, for instance, supposed to depend on the realized matching in the society reflecting the fact that people do usually care also about the partners of the others. In our model, workers' preferences are standard, while firms' preferences are defined over pairs consisting of a set of firms and a worker. Hence, our model is also broadly related to the literature on hedonic coalition formation pioneered by Drèze \& Greenberg (1980). The first ones formally introducing the model of a hedonic game were Banerjee et al. (2001) and Bogomolnaia \& Jackson (2002). Any individual in a hedonic game is supposed to have a preference over all possible coalitions she can join. Thus, at a general level, any experience dependent two-sided matching problem can be considered a hedonic game where any individually rational coalition for a firm contains a worker and a set of (other) firms, while each individually rational coalition for a worker consists of a single firm.

\footnotetext{
${ }^{1}$ For a survey on two-sided matching we refer the reader to Roth \& Sotomayor (1990).
} 


\subsection{AN EXAMPLE}

We begin with an example in order to illustrate the main ingredients of our model. As we extend preferences of firms in the above way, we have to introduce a blocking notion that slightly differs from the definition in the general model. It is also one of the main goals of this section to help the reader understand how this blocking notion works before we get into the general notation and definitions in Section 2.3.

The set of firms and workers is $F$ and $W$, respectively. Each worker is supposed to have a complete, transitive, and antisymmetric preference over $F$ and the possibility of remaining unemployed, while the strict preference of a firm $f \in F$ is generally defined over $2^{F \backslash\{f\}} \times W$ and the possibility of leaving the position vacant. This reflects the idea that firms are generally only interested by which other firms the worker has been employed.

Suppose $F=\left\{f_{1}, f_{2}\right\}$ and $W=\left\{w_{1}, w_{2}\right\}$, and let $e=\left(e\left(w_{1}\right), e\left(w_{2}\right)\right)=\left(\left\{f_{2}\right\},\left\{f_{2}\right\}\right)$ be the experience configuration indicating that both workers have been employed by firm two. The intended interpretation of $\left(\left\{f_{2}\right\}, w_{1}\right) \succ_{f_{1}}\left(\left\{f_{2}\right\}, w_{2}\right)$ is that firm $f_{1}$ compares workers $w_{1}$ and $w_{2}$ and takes into account their experience, in particular, firm $f_{1}$ strictly prefers $w_{1}$ over $w_{2}$ at experience configuration $e=\left(\left\{f_{2}\right\},\left\{f_{2}\right\}\right)$. The following example shows that a stable matching need not exist in this setup.

Example 2.1. Let $F=\left\{f_{1}, f_{2}\right\}$ and $W=\left\{w_{1}, w_{2}\right\}$. Agents' preferences are as follows:

\begin{tabular}{cccc}
$f_{1}$ & $f_{2}$ & $w_{1}$ & $w_{2}$ \\
\hline \hline$\left(\left\{f_{2}\right\}, w_{1}\right)$ & $\left(\left\{f_{1}\right\}, w_{2}\right)$ & $f_{2}$ & $f_{2}$ \\
$\left(\left\{f_{2}\right\}, w_{2}\right)$ & $\left(\left\{f_{1}\right\}, w_{1}\right)$ & $f_{1}$ & $f_{1}$ \\
$\left(\emptyset, w_{1}\right)$ & $\left(\emptyset, w_{1}\right)$ & & \\
$\left(\emptyset, w_{2}\right)$ & $\left(\emptyset, w_{2}\right)$ & & \\
\hline
\end{tabular}

Consider the configuration $e=(\emptyset, \emptyset)$ indicating that no worker has been employed by any firm. Does there exist a matching $\mu$ that is stable with respect to e when updated by the workers' corresponding mates according to $\mu$ ? Specifically, suppose the matching $\mu$ is established, such that $\mu\left(f_{1}\right)=w_{1}$ and $\mu\left(f_{2}\right)=w_{2}$ : is $\mu$ stable at the updated experience configuration $e_{\mu}=\left(\left\{f_{1}\right\},\left\{f_{2}\right\}\right)$ ? Since being unemployed is the worst option for each worker and employing a worker, independently of the configuration, is preferred by each firm over the possibility of leaving the position vacant, we conclude that $\mu$ is individually rational at $e_{\mu}$. However, $\mu$ is blocked at $e_{\mu}$ by the pair $\left(f_{2}, w_{1}\right)$ since $f_{2} \succ_{w_{1}} f_{1}=\mu\left(w_{1}\right)$ and firm $f_{2}$ strictly prefers $w_{1}$ already being employed by $f_{1}$ over $w_{2}$ not being employed by any other firm. Hence, the matching $\mu$ is not stable at $e_{\mu}$. 
The reader can easily check that the other individually rational matching $\mu^{\prime}$ defined by $\mu^{\prime}\left(f_{1}\right)=w_{2}$ and $\mu^{\prime}\left(f_{2}\right)=w_{1}$ is blocked by the pair $\left(f_{2}, w_{2}\right)$ at $e_{\mu^{\prime}}=\left(\left\{f_{2}\right\},\left\{f_{1}\right\}\right)$ and, thus, it is not stable either.

Remark 2.1. Observe that in the above example firms' preferences are monotonic and single-peaked with respect to a worker's experience, as each firm prefers a more experienced worker. Hence, we can conclude that, in general, neither monotonicity nor single-peakedness is sufficient for the existence of stable matchings at each experience configuration.

\subsection{EXPERIENCE DEPENDENT PROBLEMS}

We consider two finite sets $F$ and $W$ of agents, called "firms" and "workers", respectively. Each firm $f \in F$ has at most one position to fill and each worker can be employed by at most one firm. A matching $\mu$ is a function $\mu: F \cup W \rightarrow F \cup W$ such that $\mu(f) \in$ $W \cup\{f\}, \mu(w) \in F \cup\{w\}$, and $\mu^{2}(i)=i$ hold for $f \in F, w \in W$, and $i \in F \cup W$. The interpretation of $\mu(i)=i$ for some $i \in F \cup W$ is that the corresponding agent is unmatched under $\mu$, i.e., it is either unemployed, if $i \in W$, or the corresponding position is vacant, if $i \in F$. An experience $e: W \rightarrow 2^{F}$ assigns to each worker $w \in W$ a set of firms.

Each worker is endowed with a complete, transitive, and antisymmetric binary relation defined over $F$ and the possibility of remaining unemployed. Each firm $f \in F$ has strict preferences over $2^{F \backslash\{f\}} \times W$ and the possibility of letting the position unfilled. A profile of such preferences is denoted by $\succeq=\left(\succeq_{i}\right)_{i \in F \cup W}$. An experience dependent two-sided matching problem consists of two finite sets of agents and a strict preference profile.

Given an experience configuration $e$ and a matching $\mu$, we define the updated experience $e_{\mu}$ by $e_{\mu}(w)=e(w) \cup\{\mu(w)\}$ for each $w \in W$. We say that firm $f$ unilaterally blocks $\mu$ at $e_{\mu}$, if $f \succ_{f}\left(e_{\mu}(w) \backslash\{f\}, w\right)$. Correspondingly, worker $w$ unilaterally blocks $\mu$, if $w \succ_{w} \mu(w)$. A matching $\mu$ is individually rational at $e_{\mu}$ if it is not blocked by any agent at $e_{\mu}$. Further, we say that the pair $(f, w)$ blocks $\mu$ at $e_{\mu}$ if (1) $\left(e_{\mu}(w) \backslash\{f\}, w\right) \succ_{f}\left(e_{\mu}(\mu(f)) \backslash\{f\}, \mu(f)\right)$ and (2) $f \succ_{w} \mu(w)$. If a matching $\mu$ is individually rational at $e_{\mu}$ and there are no blocking pairs for it at $e_{\mu}$, we say that $\mu$ is stable at the experience configuration $e_{\mu}$.

\subsection{EXPERIENCE SIZE-SENSITIVITY}

As exemplified in Section 2.2, a stable matching may fail to exist when agents' preferences are experience dependent. We introduce a condition capturing the idea that firms are size-sensitive with respect to workers' past experience. We show in the next 
section that this condition assures the non-emptiness of the set of stable matchings at each experience configuration and, moreover, it enables us to fully characterize that set.

For $w \in W$ and $f \in F$, we denote by $|e(w) \backslash\{f\}|$ the experience of worker $w$ perceived by firm $f$. Notice that this leads to a different perception of the experience of a worker by different firms. The size-sensitivity condition formally stated below implements the following motivating ideas. Imagine first that each firm is paying the worker a fixed salary, provided that his experience does not exceed a firm-specific threshold $t_{f}$. In such a case the experience level does not matter at all and, thus, the firm is indifferent between two corresponding hiring decisions. This is captured in condition A1. Suppose next that salaries increase in experience if the experience level is above $t_{f}$. Assuming that a firm would rather save on salary when its targeted experience level has been reached, it seems natural that in such a case the preference should be for hiring a less experienced worker (A2). Finally, part A3 of the condition juxtaposes the idea that firms rather prefer to hire and educate a low-experienced worker instead of paying a higher salary to an over-experienced worker.

Let the notation $(F, W, \succeq, t)$ stand for an experience dependent two-sided matching problem when the threshold vector is $t=\left(t_{f}\right)_{f \in F}$ with $t_{f} \in\{0,1, \ldots,|F|-1\}$ holding for each $f \in F$.

Experience Size-Sensitivity We say that $(F, W, \succeq, t)$ is size-sensitive if for any two experience configurations $e$ and $e^{\prime}$ the following three conditions hold for each $f \in F$ and $w \in W$ :

(A1) $|e(w) \backslash\{f\}| \leq t_{f}$ and $\left|e^{\prime}(w) \backslash\{f\}\right| \leq t_{f}$ implies $(e(w), w) \sim_{f}\left(e^{\prime}(w), w\right)$;

(A2) $|e(w) \backslash\{f\}|>t_{f},\left|e^{\prime}(w) \backslash\{f\}\right|>t_{f}$, and $|e(w) \backslash\{f\}| \leq\left|e^{\prime}(w) \backslash\{f\}\right|$ implies $(e(w) \backslash\{f\}, w) \succeq_{f}\left(e^{\prime}(w) \backslash\{f\}, w\right) ;$

(A3) $|e(w) \backslash\{f\}| \leq t_{f}$ and $\left|e^{\prime}(w) \backslash\{f\}\right|>t_{f}$ implies $(e(w), w) \succ_{f}\left(e^{\prime}(w), w\right)$.

Notice that the size-sensitivity condition describes restrictions on how firms evaluate the experience levels of a particular worker without imposing any restrictions on firms' preferences over different workers (with possibly different experience levels).

\subsection{STABLE MATCHINGS}

In order to understand how experience sensitivity shapes the set of stable matchings for an experience dependent problem, we first fix a problem $(F, W, \succeq, t)$ and an experience configuration $e$. For a matching $\mu$ we define the $\mu$-based standard matching problem $\left(F, W, \succeq^{e_{\mu}}\right)$ by $\succeq_{w}^{e_{\mu}}=\succeq_{w}$ for each $w \in W$, and $w \succeq_{f}^{e_{\mu}} w^{\prime}$ if and only if $\left(e_{\mu}(w) \backslash\{f\}, w\right) \succeq_{f}\left(e_{\mu}\left(w^{\prime}\right) \backslash\{f\}, w^{\prime}\right)$ for each $f \in F$ and $w, w^{\prime} \in W$. Given the experience $e$, we have then the following connection between the set $S^{e}(F, W, \succeq, t)$ of stable 
matchings for the size sensitive experience dependent problem and the set $S^{e}\left(F, W, \succeq^{e_{\mu}}\right)$ of stable matchings for the $\mu$-based standard problem.

Theorem 2.1. Let an experience dependent matching problem $(F, W, \succeq, t)$ satisfying size-sensitivity and an experience configuration e be given. Then $\mu^{\prime} \in S^{e}(F, W, \succeq, t)$ if and only if there exists a matching $\mu$ such that $\mu^{\prime} \in S^{e}\left(F, W, \succeq^{e^{\mu}}\right)$.

Proof. Suppose first that $\mu^{\prime}$ is stable for the size-sensitive experience dependent problem $(F, W, \succeq, t)$. Take $\mu:=\mu^{\prime}$ and note that, in view of our blocking notion and the definition of firms' preferences in $\left(F, W, \succeq^{e^{\mu}}\right), \mu$ is stable for the $\mu$-based standard problem.

In order to show the reverse implication, let there exist a matching $\mu$ such that $\mu^{\prime}$ is stable for the $\mu$-based standard matching problem $\left(F, W, \succeq^{e^{\mu}}\right)$. We show that $\mu^{\prime}$ is stable for $(F, W, \succeq, t)$. The proof is divided into two steps.

Step 1: The matching $\mu^{\prime}$ is individually rational for $(F, W, \succeq, t)$.

It suffices to show that $\mu^{\prime}$ is individually rational for each $f \in F$. So, fix $f \in F$ and notice first that $\left(e_{\mu}\left(\mu^{\prime}(f)\right) \backslash\{f\}, \mu^{\prime}(f)\right) \succeq_{f} f$ clearly holds due to $\mu^{\prime}$ being stable for $\left(F, W, \succeq^{e_{\mu}}\right)$. By definition, $e_{\mu}\left(\mu^{\prime}(f)\right)=e\left(\mu^{\prime}(f)\right) \cup\left\{\mu\left(\mu^{\prime}(f)\right)\right\}$ and $e_{\mu^{\prime}}\left(\mu^{\prime}(f)\right)=$ $e\left(\mu^{\prime}(f)\right) \cup\{f\}$. Thus, $e_{\mu^{\prime}}\left(\mu^{\prime}(f)\right) \backslash\{f\} \subseteq e_{\mu}\left(\mu^{\prime}(f)\right) \backslash\{f\}$ is satisfied independently of $f$ belonging to $e\left(\mu^{\prime}(f)\right)$ or not. Consider then the following two possible cases:

(i) $\left|e_{\mu}\left(\mu^{\prime}(f)\right) \backslash\{f\}\right| \leq t_{f}$.

Clearly then, $\left|e_{\mu^{\prime}}\left(\mu^{\prime}(f)\right) \backslash\{f\}\right| \leq t_{f}$ follows by $\left|e_{\mu^{\prime}}\left(\mu^{\prime}(f)\right) \backslash\{f\}\right| \leq\left|e_{\mu}\left(\mu^{\prime}(f)\right) \backslash\{f\}\right|$. By A1, $\left(e_{\mu^{\prime}}\left(\mu^{\prime}(f)\right) \backslash\{f\}, \mu^{\prime}(f)\right) \sim_{f}\left(e_{\mu}\left(\mu^{\prime}(f)\right) \backslash\{f\}, \mu^{\prime}(f)\right)$ and, thus, by transitivity of $\succeq_{f},\left(e_{\mu^{\prime}}\left(\mu^{\prime}(f)\right) \backslash\{f\}, \mu^{\prime}(f)\right) \succeq_{f} f$ follows.

(ii) $\left|e_{\mu}\left(\mu^{\prime}(f)\right) \backslash\{f\}\right|>t_{f}$.

If $\left|e_{\mu^{\prime}}\left(\mu^{\prime}(f)\right) \backslash\{f\}\right|>t_{f}$, then $\left|e_{\mu^{\prime}}\left(\mu^{\prime}(f)\right) \backslash\{f\}\right| \leq\left|e_{\mu}\left(\mu^{\prime}(f)\right) \backslash\{f\}\right|$ implies by A2 that $\left(e_{\mu^{\prime}}\left(\mu^{\prime}(f)\right) \backslash\{f\}, \mu^{\prime}(f)\right) \succeq_{f}\left(e_{\mu}\left(\mu^{\prime}(f)\right) \backslash\{f\}, \mu^{\prime}(f)\right)$ holds and, thus, again by transitivity of $\succeq_{f},\left(e_{\mu^{\prime}}\left(\mu^{\prime}(f)\right) \backslash\{f\}, \mu^{\prime}(f)\right) \succeq_{f} f$ holds as well. If $\left|e_{\mu^{\prime}}\left(\mu^{\prime}(f)\right) \backslash\{f\}\right| \leq$ $t_{f}$, then we have $\left(e_{\mu^{\prime}}\left(\mu^{\prime}(f)\right) \backslash\{f\}, \mu^{\prime}(f)\right) \succ_{f}\left(e_{\mu}\left(\mu^{\prime}(f)\right) \backslash\{f\}, \mu^{\prime}(f)\right)$ from A3. By transitivity of $\succeq_{f},\left(e_{\mu^{\prime}}\left(\mu^{\prime}(f)\right) \backslash\{f\}, \mu^{\prime}(f)\right) \succ_{f} f$. We conclude that $\mu^{\prime}$ is individually rational for each $f \in F$ in the size-sensitive experience dependent problem.

Step 2: There are no pairs blocking $\mu^{\prime}$ in $(F, W, \succeq, t)$.

Suppose that, on the contrary, there exists a pair $(f, w)$ that blocks $\mu^{\prime}$ in $(F, W, \succeq, t)$. That is, we have

$$
\left(e_{\mu^{\prime}}(w) \backslash\{f\}, w\right) \succ_{f}\left(e_{\mu^{\prime}}\left(\mu^{\prime}(f)\right) \backslash\{f\}, \mu^{\prime}(f)\right)
$$

and $f \succ_{w} \mu^{\prime}(w)$. Since $\mu^{\prime}$ is stable for $\left(F, W, \succeq^{e^{\mu}}\right)$, we also have

$$
\left(e_{\mu}\left(\mu^{\prime}(f)\right) \backslash\{f\}, \mu^{\prime}(f)\right) \succeq_{f}\left(e_{\mu}(w) \backslash\{f\}, w\right) .
$$


Notice further that the inequalities

$$
\begin{aligned}
\left|e_{\mu}(w) \backslash\{f\}\right| & =|e(w) \cup\{\mu(w)\} \backslash\{f\}| \leq\left|e(w) \cup\left\{\mu^{\prime}(w)\right\} \backslash\{f\}\right| \\
& =\left|e_{\mu^{\prime}}(w) \backslash\{f\}\right|
\end{aligned}
$$

and

$$
\begin{aligned}
\left|e_{\mu^{\prime}}\left(\mu^{\prime}(f)\right) \backslash\{f\}\right| & =\left|e\left(\mu^{\prime}(f)\right) \cup\{f\} \backslash\{f\}\right| \leq\left|e\left(\mu^{\prime}(f)\right) \cup\left\{\mu\left(\mu^{\prime}(f)\right)\right\} \backslash\{f\}\right| \\
& =\left|e_{\mu}\left(\mu^{\prime}(f)\right) \backslash\{f\}\right|
\end{aligned}
$$

hold with the first one following from $\mu^{\prime}(w) \neq f$ and the second one by definition.

There are four cases to be considered.

Case $1\left(\left|e_{\mu}(w) \backslash\{f\}\right| \leq t_{f}\right.$ and $\left.\left|e_{\mu}\left(\mu^{\prime}(f)\right) \backslash\{f\}\right| \leq t_{f}\right)$. Notice first that, by (4) and A1,

$$
\left(e_{\mu^{\prime}}\left(\mu^{\prime}(f)\right) \backslash\{f\}, \mu^{\prime}(f)\right) \sim_{f}\left(e_{\mu}\left(\mu^{\prime}(f)\right) \backslash\{f\}, \mu^{\prime}(f)\right) .
$$

If $\left|e_{\mu^{\prime}}(w) \backslash\{f\}\right| \leq t_{f}$, then

$$
\left(e_{\mu^{\prime}}(w) \backslash\{f\}, w\right) \sim_{f}\left(e_{\mu}(w) \backslash\{f\}, w\right)
$$

follows from A1. Due to (1), (2), and transitivity of $\succeq_{f}$, we have $\left(e_{\mu}\left(\mu^{\prime}(f)\right) \backslash\{f\}, \mu^{\prime}(f)\right) \succ_{f}\left(e_{\mu^{\prime}}\left(\mu^{\prime}(f)\right) \backslash\{f\}, \mu^{\prime}(f)\right)$ in contradiction to (5).

On the other hand, if $\left|e_{\mu^{\prime}}(w) \backslash\{f\}\right|>t_{f}$ happens to be the case, we have $\left(e_{\mu^{\prime}}(w) \backslash\{f\}, w\right) \succ_{f}\left(e_{\mu}(w) \backslash\{f\}, w\right)$ following from (5), (1), (2), and transitivity of $\succeq_{f}$. Thus, we have a contradiction to $\left|e_{\mu}(w) \backslash\{f\}\right| \leq t_{f}$ and $\succeq_{f}$ satisfying A1.

Case $2\left(\left|e_{\mu}(w) \backslash\{f\}\right|>t_{f}\right.$ and $\left.\left|e_{\mu}\left(\mu^{\prime}(f)\right) \backslash\{f\}\right|>t_{f}\right)$. By (3) and A2, $\left(e_{\mu}(w) \backslash\{f\}, w\right) \succeq_{f}\left(e_{\mu^{\prime}}(w) \backslash\{f\}, w\right)$. It follows then from (1), (2), and transitivity of $\succeq_{f}$ that

$$
\left(e_{\mu}\left(\mu^{\prime}(f)\right) \backslash\{f\}, \mu^{\prime}(f)\right) \succ_{f}\left(e_{\mu^{\prime}}\left(\mu^{\prime}(f)\right) \backslash\{f\}, \mu^{\prime}(f)\right)
$$

should be the case. Notice that, in view of (4), we have that either $\left(e_{\mu^{\prime}}\left(\mu^{\prime}(f)\right) \backslash\{f\}, \mu^{\prime}(f)\right) \succeq_{f}\left(e_{\mu}\left(\mu^{\prime}(f)\right) \backslash\{f\}, \mu^{\prime}(f)\right)$ holds (by A2 and if $\left.\left|e_{\mu^{\prime}}\left(\mu^{\prime}(f)\right) \backslash\{f\}\right|>t_{f}\right)$ or that $\left(e_{\mu^{\prime}}\left(\mu^{\prime}(f)\right) \backslash\{f\}, \mu^{\prime}(f)\right) \succ_{f}\left(e_{\mu}\left(\mu^{\prime}(f)\right) \backslash\{f\}, \mu^{\prime}(f)\right)$ holds (by A3 and if $\left.\left|e_{\mu^{\prime}}\left(\mu^{\prime}(f)\right) \backslash\{f\}\right| \leq t_{f}\right)$. In both cases we have a contradiction to (7).

Case $3\left(\left|e_{\mu}(w) \backslash\{f\}\right| \leq t_{f}\right.$ and $\left.\left|e_{\mu}\left(\mu^{\prime}(f)\right) \backslash\{f\}\right|>t_{f}\right)$. If $\left|e_{\mu^{\prime}}(w) \backslash\{f\}\right| \leq t_{f}$, then (6) follows from A1 and, thus, (7) holds as well due to the same reasoning as in Case 2. Moreover, if $\left|e_{\mu^{\prime}}(w) \backslash\{f\}\right|>t_{f}$, then A3 implies $\left(e_{\mu}(w) \backslash\{f\}, w\right) \succ_{f}\left(e_{\mu^{\prime}}(w) \backslash\{f\}, w\right)$ leading again to (7) by (1), (2), and transitivity of $\succeq_{f}$. We can then precede as in Case 2 
in order to reach a contradiction to (7).

Case $4\left(\left|e_{\mu}(w) \backslash\{f\}\right|>t_{f}\right.$ and $\left.\left|e_{\mu}\left(\mu^{\prime}(f)\right) \backslash\{f\}\right| \leq t_{f}\right)$. By (3) and (4), we have that $\left|e_{\mu^{\prime}}(w) \backslash\{f\}\right|>t_{f}$ and $\left|e_{\mu^{\prime}}\left(\mu^{\prime}(f)\right) \backslash\{f\}\right| \leq t_{f}$ also holds.

By A2, $\left(e_{\mu}(w) \backslash\{f\}, w\right) \succeq_{f}\left(e_{\mu^{\prime}}(w) \backslash\{f\}, w\right)$ and by A3, $\left(e_{\mu}\left(\mu^{\prime}(f)\right) \backslash\{f\}, \mu^{\prime}(f)\right) \sim_{f}$ $\left(e_{\mu^{\prime}}\left(\mu^{\prime}(f)\right) \backslash\{f\}, \mu^{\prime}(f)\right)$ then follows. However, the latter fact together with (1), (2), and transitivity of $\succeq_{f}$ implies $\left(e_{\mu^{\prime}}(w) \backslash\{f\}, w\right) \succ_{f}\left(e_{\mu}(w) \backslash\{f\}, w\right)$, a contradiction.

The following result is then immediate.

Theorem 2.2. Let an experience dependent matching problem $(F, W, \succeq, t)$ satisfying size-sensitivity and an experience configuration e be given. Then, $S^{e}(F, W, \succeq, t)=$ $\cup_{\mu} S^{e}\left(F, W, \succeq^{e_{\mu}}\right) \neq \emptyset$.

Proof. The assertion follows directly from Theorem 2.1 together with the fact that $S^{e}\left(F, W, \succeq^{e_{\mu}}\right) \neq \emptyset$ holds for any standard matching problem $\left(F, W, \succeq^{e_{\mu}}\right)$.

\subsection{NON-EXISTENCE OF FIRM OPTIMAL STABLE MATCHINGS}

According to Theorem 2.2, the set of stable matchings given a fixed experience configuration contains matchings that are stable in the correspondingly induced standard matching problems. This fact allows for the existence of stable matchings in the experience dependent problem that are stable for some but not for all induced standard problems. It seems natural to expect that the existence of firm optimal stable matchings need not be guaranteed in our model. The next example illustrates this.

Example 2.2. The set of firms and the set of workers are $\left\{f_{1}, f_{2}\right\}$ and $\left\{w_{1}, w_{2}\right\}$. Suppose that $t_{f_{1}}=t_{f_{2}}=0$ and take the experience configuration $e=\left(\left\{f_{1}\right\},\left\{f_{2}\right\}\right)$. The preference lists for the experience dependent problem satisfying size-sensitivity are given below. For $\mu$ and $\mu^{\prime}$ defined by $\mu\left(f_{1}\right)=f_{1}, \mu\left(f_{2}\right)=w_{1}, \mu\left(w_{2}\right)=w_{2}, \mu^{\prime}\left(f_{1}\right)=w_{1}$, $\mu^{\prime}\left(f_{2}\right)=w_{2}$, firms' preferences in the corresponding $\mu$ - and $\mu^{\prime}$-based standard problems are displayed as well. 


\begin{tabular}{cccccccc}
$\succeq_{f_{1}}$ & $\succeq_{f_{2}}$ & $\succeq_{w_{1}}$ & $\succeq_{w_{2}}$ & $\succeq_{f_{1}}^{e_{\mu}}$ & $\succeq_{f_{2}}^{e_{\mu}}$ & $\succeq_{f_{1}}^{e^{\prime}}$ & $\succeq_{f_{2}}^{e^{\prime}}$ \\
\hline \hline$\left(\emptyset, w_{1}\right)$ & $\left(\emptyset, w_{1}\right)$ & $f_{1}$ & $f_{1}$ & $f_{1}$ & $w_{1}$ & $w_{1}$ & $w_{1}$ \\
$\left(\emptyset, w_{2}\right)$ & $\left(\left\{f_{1}\right\}, w_{1}\right)$ & $f_{2}$ & $f_{2}$ & $w_{1}$ & $w_{2}$ & $f_{1}$ & $w_{2}$ \\
$f_{1}$ & $\left(\emptyset, w_{2}\right)$ & $w_{1}$ & $w_{2}$ & $w_{2}$ & $f_{2}$ & $w_{2}$ & $f_{2}$ \\
$\left(\left\{f_{2}\right\}, w_{1}\right)$ & $\left(\left\{f_{1}\right\}, w_{2}\right)$ & & & & & & \\
$\left(\left\{f_{2}\right\}, w_{2}\right)$ & $f_{2}$ & & & & & & \\
\hline
\end{tabular}

The reader can easily check that $\mu$ is stable and firm optimal in the $\mu$-based standard problem, while $\mu^{\prime}$ is stable and firm optimal in the $\mu^{\prime}$-based standard problem. Moreover, neither of these matchings is stable in the corresponding other standard matching problem. In view of Theorem 2.2, both matchings are stable in the experience dependent problem with the updated experience configuration being $e_{\mu}=\left(\left\{f_{1}, f_{2}\right\},\left\{f_{2}\right\}\right)$ and $e_{\mu^{\prime}}=\left(\left\{f_{1}\right\},\left\{f_{2}\right\}\right)=e$, respectively. Notice, however, the following differences when it comes to how firms are evaluating these two stable matchings. First, firm $f_{1}$ prefers $\mu^{\prime}\left(f_{1}\right)=w_{1}$ over $\mu\left(f_{1}\right)=f_{1}$ due to $\left(e_{\mu^{\prime}}\left(w_{1}\right) \backslash\left\{f_{1}\right\}, w_{1}\right)=\left(\emptyset, w_{1}\right) \succ_{f_{1}} f_{1}$. Clearly, $f_{1}$ cannot be made better off in the experience dependent problem since $w_{1}$ is its top choice at experience $e_{\mu^{\prime}}\left(w_{1}\right)=e\left(w_{1}\right)=\left\{f_{1}\right\}$. Second, firm $f_{2}$ prefers $\mu\left(f_{2}\right)=w_{1}$ over $\mu^{\prime}\left(f_{2}\right)=$ $w_{2}$ since $\left(e_{\mu}\left(w_{1}\right) \backslash\left\{f_{2}\right\}, w_{1}\right)=\left(\left\{f_{1}\right\}, w_{1}\right) \succ_{f_{2}}\left(\emptyset, w_{2}\right)=\left(e_{\mu^{\prime}}\left(w_{2}\right) \backslash\left\{f_{2}\right\}, w_{2}\right)$. Notice that $f_{2}$ cannot be made better off in the experience dependent problem either, the reason being that the evaluation $\left(\emptyset, w_{1}\right)$ is not achievable for it due to $e_{\mu^{\prime \prime}}\left(w_{1}\right) \backslash\left\{f_{2}\right\}=\left\{f_{1}\right\}=$ $e\left(w_{1}\right)$ holding for any matching $\mu^{\prime \prime}$. We conclude that there is no stable matching for the described experience dependent problem which is unanimously the best one for all firms. 
GENDER CONSISTENT RESOLVING RULES IN MARRIAGE PROBLEMS

\subsection{INTRODUCTION}

The selection of blocking pairs to be matched plays an important role in the study of mechanisms converting arbitrary matchings into stable ones as shown in the seminal works of Knuth (1976) and Roth \& Vande Vate (1990). The latter work considers sequences of matchings, where each matching is obtained from the previous one by satisfying a single blocking pair, and introduces an algorithm which selects in a specific way the blocking pair to be satisfied at each iteration until a stable matching is reached. ${ }^{1}$ The focus on matching a single blocking pair at each iteration of an algorithm turned out to be fruitful also with respect to the original Gale-Shapley procedure (cf. Gale \& Shapley, 1962) for showing the existence of a stable matching. For instance, McVitie \& Wilson (1970) have modified Gale and Shapley's algorithm by letting at each iteration only one man propose to the woman he prefers most among those who have not yet rejected him.

The single-proposal variant of the Gale-Shapley algorithm and the Roth-Vande Vate algorithm were shown in Abeledo \& Rothblum (1995) to share a common feature. More precisely, the common feature is shared by the corresponding resolving rules, that is, by the rules applied in both algorithms for the selection of a blocking pair to be matched from the set of all pairs blocking a given matching. These resolving rules turn out to follow a pre-specified linear order over the set of acceptable pairs which is gender consistent, that is, the linear order is consistent with the preferences of either all men or all women (see Sections 3.2 and 3.3 for the precise definitions).

In this chapter ${ }^{2}$ we provide an axiomatic foundation of gender consistent resolving rules. In doing so, we focus on male consistency, that is, the corresponding rule shall respect the preferences of all men. When defining the domain on which a resolving rule is supposed to work, we consider preference profiles differing only with respect to women's preferences and matchings that are individually rational (but not stable) at such profiles. Given such a domain, a resolving rule then assigns, to each preference profile and matching, a pair that blocks the matching at that preference profile. It is worth

\footnotetext{
${ }^{1}$ As shown by Ma (1996), the mechanism suggested by Roth \& Vande Vate (1990) does not always reach all stable matchings.

${ }^{2}$ This chapter is based on Dimitrov et al. (2018).
} 
mentioning that the above domain restrictions are in line with Abeledo \& Rothblum (1995), where male consistency is defined with respect to a fixed profile of men's preferences and matchings are always supposed to be individually rational.

The algorithms based on gender consistent resolving rules can generally be interpreted as describing different ways for assigning a stable matching to any (individually rational) initial matching. In that sense, our work can be seen in the same vain as the axiomatically driven papers of Kojima \& Manea (2010) and Morrill (2013). The rules characterized in these papers are based on the deferred acceptance algorithm applied in the context of object allocation problems. In contrast, and to the best of our knowledge, the present paper is the first one to offer an axiomatic characterization of a whole class of rules in the context of two-sided matching.

We formulate two requirements - an independence axiom and a top man optimality condition - and impose them on a resolving rule. The independence axiom works on different pairs consisting of a preference profile and a matching, and forces a resolving rule to select the same outcome, provided that the corresponding sets of blocking pairs do coincide. As for our second requirement, we slightly modify the notion of domination among blocking pairs introduced in Klijn \& Massó (2003), and require in the top man optimality condition any resolving rule outcome to be undominated.

The chapter is organized as follows. Section 3.2 contains basic notation and definitions, as well as the precise formulations of the proposed axioms. We then show in Section 3.3 that a resolving rule satisfying these axioms must be male consistent. Finally, we discuss independence of the axioms.

\subsection{SETUP AND AXIOMS}

We consider the standard two-sided one-to-one matching model introduced in Gale \& Shapley (1962), in which there are two finite sets $M$ and $W$ of agents, called "men" and "women", respectively. These two sets will be kept fixed throughout the paper with each agent being endowed with a complete, transitive, and antisymmetric binary relation over the agents from the opposite sex and the possibility of remaining single. For instance, $w \succ_{m} w^{\prime}$ expresses the fact that man $m \in M$ prefers woman $w \in W$ to woman $w^{\prime} \in W$; we write $w \succeq_{m} w^{\prime}$ whenever either $w \succ_{m} w^{\prime}$ or $w=w^{\prime}$ holds. A preference profile is denoted by $\succeq=\left(\succeq_{i}\right)_{i \in M \cup W}$, and we say that a pair $(m, w) \in M \times W$ is acceptable at $\succeq$ if $w \succ_{m} m$ and $m \succ_{w} w$ holds. The set of all acceptable pairs at $\succeq$ is denoted by $A \succeq$.

A matching $\mu$ is a function $\mu: M \cup W \rightarrow M \cup W$ such that $\mu(m) \in W \cup\{m\}, \mu(w) \in$ $M \cup\{w\}$, and $\mu^{2}(i)=i$ hold for $m \in M, w \in W$, and $i \in M \cup W$. The interpretation of $\mu(i)=i$ for some $i \in M \cup W$ is that the corresponding agent is single under $\mu$. If $\mu(i)=i$ holds for each $i \in M \cup W$, we say that the corresponding matching is empty 
and denote it throughout the paper by $\mu_{0}$. A matching $\mu$ is called stable at $\succeq$ if (1) it consists of either singletons or acceptable pairs at $\succeq$ (individual rationality), and (2) there are no blocking pairs at $\succeq$ for it, i.e., there is no pair $(m, w)$ such that $w \succ_{m} \mu(m)$ and $m \succ_{w} \mu(w)$. The set of blocking pairs for a matching $\mu$ at a profile $\succeq$ is denoted by $B \succeq(\mu)$.

Let $\mathcal{M}$ stand for the set of all matchings, $\left(\succeq_{m}\right)_{m \in M}$ be a fixed profile of men's preferences, and $\mathcal{R}$ be the largest collection of preference profiles for which $\succeq^{\prime} \in \mathcal{R}$ implies $\succeq_{m}^{\prime}=\succeq_{m}$ for each $m \in M$. We collect in the set $\mathcal{D}$ all $(\succeq, \mu) \in \mathcal{R} \times \mathcal{M}$ such that $\mu$ is individually rational but not stable at $\succeq$. A resolving rule $g$ is defined on $\mathcal{D}$ and assigns a pair $(m, w) \in B^{\succeq}(\mu)$ to each $(\succeq, \mu) \in \mathcal{D}$. Notice then that, for each $(\succeq, \mu) \in \mathcal{D}$, we have $B^{\succeq}(\mu) \subseteq A^{\succeq}$.

The first axiom we impose on a resolving rule is a standard independence requirement. In our context it captures the idea that, when comparing two different pairs of preference profiles and matchings, the outcome of a resolving rule remains the same if the corresponding sets of blocking pairs are related by set inclusion as specified below. As a consequence, the axiom allows us to conclude that it is the set of blocking pairs that matters when a rule satisfying the axiom selects a particular pair. Notice additionally that the corresponding set of blocking pairs is well defined and non-empty for any matching and preference profile in the domain we consider. Thus, a resolving rule can in fact be seen as selecting a single blocking pair from a set of blocking pairs; our independence requirement correctly supports this particular interpretation.

Independence of Irrelevant Alternatives (IIA): If $B^{\succeq}(\mu) \subseteq B^{\succeq^{\prime}}\left(\mu^{\prime}\right)$ for some $(\succeq$ $, \mu),\left(\succeq^{\prime}, \mu^{\prime}\right) \in \mathcal{D}$, then $g\left(\succeq^{\prime}, \mu^{\prime}\right) \in B^{\succeq}(\mu)$ implies $g(\succeq, \mu)=g\left(\succeq^{\prime}, \mu^{\prime}\right)$.

In order to introduce our second axiom, let us consider $(\succeq, \mu) \in \mathcal{D}$ with $(m, w)$, $\left(m, w^{\prime}\right) \in B^{\succeq}(\mu)$. Moreover, let man $m$ be the top man for both $w$ and $w^{\prime}$ at $\succeq$; that is, $m \succeq_{w^{\prime \prime}} m^{\prime \prime}$ holds for $w^{\prime \prime} \in\left\{w, w^{\prime}\right\}$ and all $m^{\prime \prime} \in M$. We deem then $g$ top man dominated at $(\succeq, \mu)$ if $g(\succeq, \mu)=(m, w)$ and $w^{\prime} \succ_{m} w$. A resolving rule $g$ is top man optimal if it is not top man dominated at any $(\succeq, \mu) \in \mathcal{D}$.

Top Man Optimality (TMO): $g$ is top man optimal.

As to understand the power of TMO, suppose that $g(\succeq, \mu)=(m, w)$ holds for some $(\succeq, \mu) \in \mathcal{D}$. Notice then that $g$ being top man optimal does not necessarily imply that $m$ is a top man at $\succeq$ for $w$. It rather says that, provided $m$ is the top man for $w$ at $\succeq$, there is no other woman $w^{\prime}$ with the same top man at $\succeq$ such that $\left(m, w^{\prime}\right) \in B^{\succeq}(\mu)$ and $w^{\prime} \succ_{m} w^{3}$

\footnotetext{
${ }^{3}$ Klijn \& Massó (2003) introduce weak stability for the marriage model that allows for the existence of weak blocking pairs, and show that Zhou's bargaining set for their context does coincide with the set of weakly stable and weakly efficient matchings. More precisely, a pair $(m, w) \in B^{\succeq}(\mu)$ is called weak if there exists either a woman $w^{\prime}$ with $\left(m, w^{\prime}\right) \in B^{\succeq}(\mu)$ and $w^{\prime} \succ_{m} w$, or a man $m^{\prime}$ with $\left(m^{\prime}, w\right) \in B^{\succeq}(\mu)$
} 


\subsection{CHARACTERIZATION}

In this section we provide our characterization result by first showing that the IIA axiom forces a resolving rule to become dependent on a linear order over acceptable pairs (Proposition 3.1). We then prove that adding TMO to IIA implies that the constructed linear order becomes male consistent (Proposition 3.2).

Let us start with the simple observation that the set $\mathcal{R}$ of preference profiles we consider contains a profile $\succeq^{*}$ at which $m \succ_{w}^{*} w$ holds for any $m \in M$ and $w \in W$. Clearly then, $A \succeq \subseteq A^{\succeq *}$ holds for each $\succeq \in \mathcal{R}$. The following lemma will prove useful subsequently.

Lemma 3.1. Let $A \subseteq A^{\succeq^{*}}$. Then there exists $(\succeq, \mu) \in \mathcal{D}$ such that $(m, w) \in B^{\succeq}(\mu)$ if and only if $(m, w) \in A$.

Proof. Take $\mu=\mu_{0}$ and notice that $\mu_{0}$ is individually rational at any preference profile. Recall that men's preferences are fixed and take $\succeq \in \mathcal{R}$ to be such that, for $m \in M$ and $w \in W, m \succeq_{w} w$ if and only if $(m, w) \in A$. By completeness of women's preferences, $\left(m^{\prime}, w\right) \in A^{\succeq^{*}} \backslash A$ for some $m^{\prime} \in M$ implies $w \succ_{w} m^{\prime}$. We conclude that $(m, w) \in$ $B^{\succeq}\left(\mu_{0}\right)$ if and only if $(m, w) \in A$.

Our first result shows that rules satisfying IIA and those that are order dependent turn out to be equivalent. More precisely, a resolving rule $g$ is order dependent if there exists a linear order $\unrhd$ over $A^{\succeq^{*}}$ such that, for each $(\succeq, \mu) \in \mathcal{D}, g(\succeq, \mu) \unrhd(m, w)$ holds for each $(m, w) \in B^{\succeq}(\mu)$. We write here $(m, w) \unrhd\left(m^{\prime}, w^{\prime}\right)$ to express the fact that either $(m, w) \triangleright\left(m^{\prime}, w^{\prime}\right)$ or $(m, w)=\left(m^{\prime}, w^{\prime}\right)$ holds.

Proposition 3.1. A resolving rule satisfies IIA if and only if it is order dependent.

Proof. We first show that if a resolving rule $g$ is order dependent with respect to some linear order $\unrhd$ over $A^{\succeq^{*}}$, then it satisfies IIA. For this, take $(\succeq, \mu),\left(\succeq^{\prime}, \mu^{\prime}\right) \in \mathcal{D}$ and suppose that $B^{\succeq}(\mu) \subseteq B^{\succeq^{\prime}}\left(\mu^{\prime}\right)$. By the order dependence of $g, g\left(\succeq^{\prime}, \mu^{\prime}\right) \unrhd(m, w)$ holds for all $(m, w) \in B^{\succeq^{\prime}}\left(\mu^{\prime}\right) \supseteq B^{\succeq}(\mu)$. Clearly then, $g\left(\succeq^{\prime}, \mu^{\prime}\right) \in B^{\succeq}(\mu)$ implies $g(\succeq, \mu)=g\left(\succeq^{\prime}, \mu^{\prime}\right)$ and thus, $g$ satisfies IIA.

Suppose now that a resolving rule $g$ satisfies IIA. As to show the order dependence of $g$, we shall construct a linear order $\unrhd$ over $A^{\succeq^{*}}$ and then prove that $g$ is order dependent with respect to $\unrhd$.

Consider the set $A \succeq^{*}$ and notice that by Lemma 3.1 there exists $(\succeq, \mu) \in \mathcal{D}$ such that $B^{\succeq}(\mu)=A^{\succeq^{*}}$. Set then $g(\succeq, \mu) \triangleright(m, w)$ to hold for all $(m, w) \in A^{\succeq^{*}} \backslash\{g(\succeq, \mu)\}$. Consider next the set $A^{\prime}=A^{\succeq^{*}} \backslash\{g(\succeq, \mu)\}$ and apply again Lemma 3.1 to conclude

and $m^{\prime} \succ_{w} m$. Notice that our dominance notion is stronger and thus, the corresponding TMO condition allows for the selection of weak blocking pairs. 
the existence of $\left(\succeq^{\prime}, \mu^{\prime}\right) \in \mathcal{D}$ with $B^{\succeq^{\prime}}\left(\mu^{\prime}\right)=A^{\prime}$. Set then $g\left(\succeq^{\prime}, \mu^{\prime}\right) \triangleright\left(m^{\prime}, w^{\prime}\right)$ for all $\left(m^{\prime}, w^{\prime}\right) \in A^{\prime} \backslash\left\{g\left(\succeq^{\prime}, \mu^{\prime}\right)\right\}$. Continue in this way until the set $A^{\succeq^{*}}$ is exhausted. Clearly, by its very construction, the binary relation $\unrhd$ is complete, transitive, and antisymmetric and thus, a linear order over $A^{\succeq^{*}}$.

Let us now show that $g$ is order dependent with respect to $\unrhd$. Take $(\succeq, \mu) \in \mathcal{D}$ and let $(m, w) \unrhd\left(m^{\prime}, w^{\prime}\right)$ hold for all $\left(m^{\prime}, w^{\prime}\right) \in B^{\succeq}(\mu)$. We show that $g(\succeq, \mu)=(m, w)$ should follow. For this, let

$$
A^{\prime \prime}=\left\{\left(m^{\prime \prime}, w^{\prime \prime}\right) \in A^{\succeq^{*}}:(m, w) \unrhd\left(m^{\prime \prime}, w^{\prime \prime}\right)\right\} .
$$

By Lemma 3.1, there exists $\left(\succeq^{\prime \prime}, \mu^{\prime \prime}\right) \in \mathcal{D}$ such that $B^{\succeq^{\prime \prime}}\left(\mu^{\prime \prime}\right)=A^{\prime \prime}$. By the construction of $\unrhd$ then, $g\left(\succeq^{\prime \prime}, \mu^{\prime \prime}\right)=(m, w)$ follows. Finally, from $(m, w) \in B^{\succeq}(\mu) \subseteq A^{\prime \prime}$ and IIA, we conclude that $g(\succeq, \mu)=(m, w)$ should hold.

We are now ready for our main result that characterizes gender consistent resolving rules. These rules were introduced in Abeledo \& Rothblum (1995) as a special class of order dependent resolving rules for which the corresponding linear order over acceptable pairs is either male consistent or female consistent. As already mentioned in the Introduction, we focus on male consistency.

For $\succeq \in \mathcal{R}$, we say that a linear order $\unrhd$ over $A^{\succeq^{*}}$ is male consistent if for any $(m, w),\left(m, w^{\prime}\right) \in A^{\succeq^{*}},(m, w) \unrhd\left(m, w^{\prime}\right)$ if and only if $w \succeq_{m} w^{\prime}$. Notice that, as men's preferences remain the same at each $\succeq \in \mathcal{R}$, this definition ensures male consistency of $\unrhd$ with respect to any $\succeq \in \mathcal{R}$. We say finally that a resolving rule $g$ is male consistent if it is order dependent with respect to some male consistent linear order over $A^{\succeq^{*}}$.

Our main result shows that any order dependent rule that is top man optimal must be male consistent.

Proposition 3.2. A resolving rule satisfies IIA and TMO if and only if it is male consistent.

Proof. Let $g$ be order dependent with respect to some male consistent linear order $\unrhd$ over $A \succeq^{*}$. The fact that $g$ satisfies IIA was shown in the proof of Proposition 3.1. On the other hand, $g$ violating TMO would imply the existence of $(\succeq, \mu) \in \mathcal{D}$ and $\left(m, w^{\prime}\right) \in B^{\succeq}(\mu)$ such that $(m, w)=g(\succeq, \mu)$ and $w^{\prime} \succ_{m} w$. By $\unrhd$ being male consistent, $\left(m, w^{\prime}\right) \triangleright(m, w)$ should be the case. Thus, we reach a contradiction to $g(\succeq, \mu)=(m, w)$ and $g$ being order dependent with respect to $\unrhd$. We conclude that $g$ satisfies TMO as well.

Suppose now that a resolving rule $g$ satisfies IIA and TMO. Consider the linear order $\unrhd$ constructed in the proof of Proposition 3.1 and recall that $g$ is then order dependent with respect to $\unrhd$. We show that TMO restricts $\unrhd$ to be male consistent.

For the sake of contradiction, assume that $\unrhd$ violates male consistency. Let $(m, w)$ be a pair such that there is $w^{\prime} \in W$ with $w^{\prime} \succ_{m} w \succ_{m} m$ and $(m, w) \triangleright\left(m, w^{\prime}\right)$ (as $\unrhd$ is 
assumed to violate male consistency, such a pair exists). Let $\succeq \in \mathcal{R}$ be a profile such that $(m, w)$ and $\left(m, w^{\prime}\right)$ are the only acceptable pairs and women's preferences be as follows:

- $m \succ_{w} w \succ_{w} m^{\prime}$ and $m \succ_{w^{\prime}} w^{\prime} \succ_{w^{\prime}} m^{\prime}$ for all $m^{\prime} \in M \backslash\{m\}$; and

- $w^{\prime \prime} \succ_{w^{\prime \prime}} m^{\prime}$ for all $w^{\prime \prime} \in W \backslash\left\{w, w^{\prime}\right\}$ and $m^{\prime} \in M$.

Consider finally the matching $\mu_{0}$ and notice that $B^{\succeq}\left(\mu_{0}\right)=\left\{(m, w),\left(m, w^{\prime}\right)\right\}$. By $g$ being order dependent with respect to $\unrhd$, it holds that $g\left(\succeq, \mu_{0}\right)=(m, w)$. However, this implies that $g$ is top man dominated at $\left(\succeq, \mu_{0}\right)$, a contradiction.

Notice that the IIA axiom relates resolving rule outcomes at different pairs of preference profiles and matchings, while the TMO axiom is a condition imposed on the outcome of a resolving rule at a given profile-matching pair. Thus, it is natural to expect that these axioms are independent. Our next two examples do indeed show this fact. More precisely, we provide below two particular resolving rules each of which satisfying one of the axioms but not the other. In both examples, $M=\left\{m_{1}, m_{2}\right\}$ and $W=\left\{w_{1}, w_{2}\right\}$.

We start by constructing a resolving rule $g_{1}$ fulfilling IIA but violating TMO. Let men's preferences be fixed at $w_{1} \succ_{m_{1}} w_{2} \succ_{m_{1}} m_{1}$ and $m_{2} \succ_{m_{2}} w_{1} \succ_{m_{2}} w_{2}$. Consider the preference profile $\succeq \in \mathcal{R}$ at which women's preferences are as follows: $m_{1} \succ_{w_{1}}$ $w_{1} \succ_{w_{1}} m_{2}$ and $m_{1} \succ_{w_{2}} w_{2} \succ_{w_{2}} m_{2}$. Let $g_{1}$ be order dependent with respect to the following linear order $\unrhd_{1}$ over the set $A^{\succeq^{*}}=A^{\succeq}=\left\{\left(m_{1}, w_{1}\right),\left(m_{1}, w_{2}\right)\right\}:\left(m_{1}, w_{2}\right) \triangleright_{1}$ $\left(m_{1}, w_{1}\right)$. By Proposition 3.1, $g_{1}$ satisfies IIA. Take $\mu_{0}$ and notice that $B \succeq\left(\mu_{0}\right)=A^{\succeq}$ and $g_{1}\left(\succeq, \mu_{0}\right)=\left(m_{1}, w_{2}\right)$ holds. Clearly then, $g_{1}$ is top man dominated at $\left(\succeq, \mu_{0}\right)$.

Let us now construct a resolving rule $g_{2}$ satisfying TMO but not IIA. Fix men's preferences as follows: $w_{1} \succ_{m_{1}} m_{1} \succ_{m_{1}} w_{2}$ and $w_{1} \succ_{m_{2}} m_{2} \succ_{m_{2}} w_{2}$. Consider the profiles $\succeq^{\prime}, \succeq^{\prime \prime} \in \mathcal{R}$ at which $w_{1}$ 's preferences are $m_{1} \succ_{w_{1}}^{\prime} m_{2} \succ_{w_{1}}^{\prime} w_{1}$ and $m_{2} \succ_{w_{1}}^{\prime \prime}$ $m_{1} \succ_{w_{1}}^{\prime \prime} w_{1}$, respectively. Let $g_{2}$ be any resolving rule satisfying $g_{2}\left(\succeq^{\prime}, \mu_{0}\right)=\left(m_{1}, w_{1}\right)$ and $g_{2}\left(\succeq^{\prime \prime}, \mu_{0}\right)=\left(m_{2}, w_{1}\right)$. Clearly then, $g_{2}$ vacuously satisfies TMO since only $w_{1}$ can form blocking pairs with $m_{1}$ and $m_{2}$. Notice however, that $g_{2}$ violates IIA since $B^{\succeq^{\prime}}\left(\mu_{0}\right)=B^{\succeq^{\prime \prime}}\left(\mu_{0}\right)$ but $g_{2}\left(\succeq^{\prime}, \mu_{0}\right) \neq g_{2}\left(\succeq^{\prime \prime}, \mu_{0}\right)$. We conclude that IIA and TMO do not imply each other. 


\section{CONDORCET CONSISTENCY AND THE STRONG NO SHOW}

PAR A DOXES

\subsection{INTRODUCTION}

Within a democratic voting system it should be in the own interest of voters not to abstain from voting. Abstaining from voting, however, can be rational even in voting systems that are often regarded as truly democratic. A recent example is the Dutch referendum for the EU Association Treaty with the Ukraine on April 6, 2016. A majority voted against this treaty, but the total voter turnout was hardly more than $2 \%$ above the minimally required 30\%: if some of the 'yes' voters would have stayed at home then the referendum would have been invalid and the 'no' to the treaty avoided.

Another natural and desirable property of a voting system is Condorcet Consistency (CC) (cf. de Condorcet, 1785): if there is an alternative (candidate, political party, law, etc.) that is ranked above any other alternative by some (weak) majority of the voters, then that alternative should be chosen. Also this property is often not fulfilled. Again in the Netherlands, van Deemen \& Vergunst (1998) provide evidence that D66 (a progressive liberal party) may have been the Condorcet winner in several consecutive Dutch elections for Parliament, but it never won those elections and, consequently, could never take the lead in the formation of a new government.

In this chapter we study voting correspondences which combine the two desirable conditions above: they should be Condorcet Consistent and not admit situations where voters are better off by abstaining. The latter will mean, more precisely, that it should never happen that an additional voter, by ranking a winning alternative first turns this alternative into a losing one or, by ranking a losing alternative last, turns this into a winning alternative. In the two-alternative referendum on the Ukraine Association treaty mentioned above both these situations occurred. In Nurmi (2002) and Pérez (2001) these phenomena are referred to as the positive and negative strong no show paradoxes, respectively. Thus, we are looking for Condorcet Consistent voting correspondences that avoid both these paradoxes, and we call the avoidance of these paradoxes the Top Property (TP) and the Bottom Property (BP), respectively.

Although this topic has been studied before, to the best of our knowledge we are the first to obtain positive and fairly complete results. As one of the main results in this chapter we identify the maximal Condorcet Consistent voting correspondence $H$ that 
satisfies the Top Property, that is: each Condorcet Consistent voting correspondence satisfying the Top Property must be contained in $H$ and, a fortiori, $H$ is the union of all smaller voting correspondences with the two properties. We also show that this result remains true if we add the Bottom Property, but not if we replace the Top Property by the Bottom Property. The Minimax Rule, in particular, is contained in $H$. Furthermore, we show that also voting functions (single-valued or resolute voting correspondences) that are Condorcet Consistent and rule out both paradoxes must select from $H$, and we exhibit several ways in which this can or cannot be done. Finally, we consider a weaker version of Condorcet Consistency, meaning that the voting correspondence should pick the (unique) strong Condorcet winner if there is one, and discuss which of our results remain valid.

We next discuss the main related literature. The Bottom Property is sufficient to rule out the no show paradox as formulated in Brams \& Fishburn (1983). Moulin (1988) shows that if there are at least four alternatives and at least twenty-five voters, no Condorcet Consistent voting function can satisfy a condition called 'participation': this condition requires that no voter can be worse off by voting than by abstention. The participation condition rules out the no show paradox as formulated in Moulin (1988). This no show paradox is weaker (hence, occurs more often) than the combination of the two strong versions that we consider and, consequently, the participation condition in Moulin (1988) is stronger than the combination of the Top Property and the Bottom Property. The version of Condorcet Consistency considered in Moulin (1988), on the other hand, is our weaker version of Condorcet Consistency to be discussed in Section 4.5. For extensions and strengthenings of the results in Moulin (1988) on the (weak) no show paradox see Jimeno et al. (2009), Brandt et al. (2017), and Núñez \& Sanver (2017). On the strong no show paradoxes see Pérez (2001), Felsenthal \& Tideman (2013), Duddy (2014), and Felsenthal \& Nurmi (2016). In particular, Pérez (2001) shows that all Condorcet Consistent voting correspondences satisfying some weak domination properties are affected by the strong no show paradoxes. Our Top and Bottom Properties correspond to what is called Positive and Negative Involvement in Pérez (2001), while the Condorcet Consistency considered in that paper is equivalent to the one discussed in Section 4.5. In order to derive the maximal correspondence satisfying CC, TP and $\mathrm{BP}$, we make use of a technical condition which is related to the one in Lemma 1 of Pérez (2001). Contrary to this work and to Moulin (1988) we obtain positive (existence) instead of negative (non-existence) results.

Felsenthal \& Tideman (2013) examine voting functions based on five well known voting correspondences with respect to the strong no show paradoxes. Felsenthal $\&$ Nurmi (2016) extend the work of Felsenthal \& Tideman (2013) by illustrating and analyzing the susceptibility of nine voting functions to the positive and negative strong 
no show paradoxes. Duddy (2014) allows weak preference rankings and shows that then every Condorcet consistent voting function must exhibit both paradoxes if there are at least four alternatives. Different from Duddy (2014) we consider linear orderings, that is, strict rankings. Our work is mainly in line with the work of Pérez (2001), Felsenthal \& Tideman (2013) and Felsenthal \& Nurmi (2016). In particular, Pérez (2001) and Felsenthal \& Nurmi (2016) argue that the Minimax Rule (cf. Black, 1958; Kramer, 1977; Simpson, 1969) is the only voting correspondence presented in the literature so far that is not affected by any of the two paradoxes - the Minimax Rule chooses those alternatives for which a minimal number of voters rank some other alternative higher, and as already mentioned it is contained in our maximal voting correspondence $H$. They also show that Young's Rule (cf. Young, 1977) is not affected by the negative strong no show paradox. Young's Rule chooses those alternatives for which to become a (weak) Condorcet winner, the minimal number of voters has to be removed from the profile.

The chapter is organized as follows. In Section 4.2 we provide basic concepts and definitions, and in Section 4.3 we consider voting correspondences. Our main result is Theorem 4.1, which states that the correspondence $H$ alluded to above is the maximal voting correspondence that is Condorcet Consistent and satisfies both the Top Property and Bottom Property and there is no strict subcorrespondence of it that contains all voting correspondences with these three properties, which are unequal to the maximal one. In Section 4.4 we consider voting functions with these properties, which are necessarily selections from $H$. We may, in particular, choose from the Minimax Rule according to a fixed tie-breaking ordering, but this result does not extend to $H$. In Section 4.5 we consider a weaker version of Condorcet Consistency and elaborate on which of our results remain valid. Section 4.6 concludes. Some of the proofs are relegated to the Appendix.

\subsection{BASIC DEFINITIONS}

We let $A$ denote the finite set of alternatives. The set of potential voters is $\mathbb{N}$. A ranking is a linear ordering (reflexive, complete, transitive, and antisymmetric) over $A$, and $L$ denotes the set of rankings. For a finite set $N \subseteq \mathbb{N}, R^{N}=\left(R^{i}\right)_{i \in N} \in L^{N}$ is called a profile. For $j \in \mathbb{N} \backslash N$ and $R^{j} \in L$ we typically denote by $\left(R^{N}, R^{j}\right)$ a profile where each $i \in N$ has ranking $R^{i}$ and $j$ has ranking $R^{j}$. Also, for $R^{\prime} \in L,\left(R^{N}, R^{\prime}\right)$ denotes a profile for $N$ and an additional voter with ranking $R^{\prime}$. For a ranking $R$ we denote by $t(R)$ its top alternative and by $b(R)$ its bottom alternative, that is, $t(R) R x R b(R)$ for all $x \in A$.

For two distinct alternatives $x$ and $y$ and a profile $R^{N}$,

$$
n_{x y}\left(R^{N}\right)=\left|\left\{i \in N: x R^{i} y\right\}\right|-\left|\left\{i \in N: y R^{i} x\right\}\right|
$$


denotes the net number of voters ranking $x$ over $y$. Clearly, $n_{x y}\left(R^{N}\right)=-n_{y x}\left(R^{N}\right)$. The number

$$
m_{x}\left(R^{N}\right)=\max _{y \in A \backslash\{x\}} n_{y x}\left(R^{N}\right)
$$

is called the (maximal) resistance against $x$. Note that $n_{x y}\left(R^{N}\right)$ and $m_{x}\left(R^{N}\right)$ have the same parity (odd or even) as $|N|$.

An alternative $x$ is a (weak) Condorcet winner at $R^{N}$ if $m_{x}\left(R^{N}\right) \leq 0$ or, equivalently, $n_{x y}\left(R^{N}\right) \geq 0$ for all $y \in A \backslash\{x\}$. The (possibly empty) set of Condorcet winners is denoted by $\mathcal{C}\left(R^{N}\right)$, hence

$$
\mathcal{C}\left(R^{N}\right)=\left\{x \in A: m_{x}\left(R^{N}\right) \leq 0\right\}
$$

Remark 4.1. Clearly, if $m_{x}\left(R^{N}\right)<0$ for some $x \in A$, then $n_{y x}\left(R^{N}\right)<0$ for all $y \in$ $A \backslash\{x\}$, which in turn implies $n_{x y}\left(R^{N}\right)>0$ and thus $m_{y}\left(R^{N}\right)>0$ for all $y \in A \backslash\{x\}$. Therefore, $\mathcal{C}\left(R^{N}\right)=\{x\}$. In this case, $x$ is the unique Condorcet winner, usually called the strong Condorcet winner.

A voting correspondence $C$ assigns to each profile $R^{N}$ for every finite $N \subseteq \mathbb{N}$ a nonempty subset $C\left(R^{N}\right)$ of $A$, and a voting function $F$ assigns to each profile $R^{N}$ an alternative $F\left(R^{N}\right) \in A$. If $F\left(R^{N}\right) \in C\left(R^{N}\right)$ for each profile $R^{N}$, then we call (voting function) $F$ a selection from (voting correspondence) $C$.

\subsection{CONDORCET CONSISTENCY, TOP PROPERTY, AND BOTTOM PROPERTY FOR VOTING CORRESPONDENCES}

Let $C$ be a voting correspondence. The following properties are of central interest in this chapter.

Condorcet Consistency (CC) For every profile $R^{N}$ such that $\mathcal{C}\left(R^{N}\right) \neq \emptyset, C\left(R^{N}\right)=$ $\mathcal{C}\left(R^{N}\right)$.

Top Property (TP) For every profile $R^{N}$, every $j \in \mathbb{N} \backslash N$, every $x \in A$, and every $R^{j}$ with $t\left(R^{j}\right)=x$, if $x \in C\left(R^{N}\right)$, then $x \in C\left(R^{N}, R^{j}\right)$.

Bottom Property (BP) For every profile $R^{N}$, every $j \in \mathbb{N} \backslash N$, every $x \in A$, and every $R^{j}$ with $b\left(R^{j}\right)=x$, if $x \notin C\left(R^{N}\right)$, then $x \notin C\left(R^{N}, R^{j}\right)$.

Condorcet Consistency says that if there exist Condorcet winners, then exactly those should be chosen. The two other properties guarantee that the following two situations cannot occur. First, if $x$ is chosen and an additional voter ranks $x$ at top, then $x$ is no longer chosen; second, if $x$ is not chosen and an additional voter ranks $x$ at bottom, then $x$ is chosen. Thus, in theses cases, the additional voter would be better off by abstention. The Top Property and the Bottom Property prevent exactly these two no show paradoxes. 
A main goal of this chapter is to investigate which voting correspondences satisfy all three properties. As a starting point we consider the Minimax Rule, which dates back to Black (1958), Simpson (1969), and Kramer (1977). The Minimax Rule $M$ is defined by

$$
M\left(R^{N}\right)=\left\{x \in A: m_{x}\left(R^{N}\right) \leq m_{y}\left(R^{N}\right) \text { for all } y \in A\right\},
$$

hence it chooses those alternatives against which resistance is minimal.

The following result is already known (e.g., Felsenthal \& Nurmi (2016); Pérez (2001)), but for completeness we provide a proof in the Appendix.

Proposition 4.1. $M$ satisfies $C C, T P$, and $B P$.

We first concentrate on CC and TP, and investigate if it is possible to choose alternatives additional to those in $M$ without violating TP. The following example may be instructive as it gives insights when choosing additional alternatives is possible and when it is not.

Example 4.1. Let $A=\{a, b, c\}$ and consider the ten voter profile $R^{N}$ given by

\begin{tabular}{lll}
3 & 3 & 4 \\
\hline$a$ & $b$ & $c$ \\
$b$ & $c$ & $a$ \\
$c$ & $a$ & $b$
\end{tabular}

where the first column indicates that three voters have ranking $R$ with aRbRc, also denoted as $R=a b c$; etc. The table of pairwise comparisons is

\begin{tabular}{c|ccc} 
& $a$ & $b$ & $c$ \\
\hline$a$ & - & 7 & 3 \\
$b$ & 3 & - & 6 \\
$c$ & 7 & 4 & -
\end{tabular}

For instance, the number 7 in row a and column $b$ means that seven voters rank $a$ above $b$. Hence $n_{a b}\left(R^{N}\right)=7-3=4, n_{a c}\left(R^{N}\right)=-4$, and $n_{b c}\left(R^{N}\right)=2$. Then $m_{a}\left(R^{N}\right)=m_{b}\left(R^{N}\right)=4$ and $m_{c}\left(R^{N}\right)=2$, so that $\mathcal{C}\left(R^{N}\right)=\emptyset$ and $M\left(R^{N}\right)=\{c\}$.

Could, additionally to $c$, also a be chosen? If we add two voters who rank a on top and c second, then c becomes a Condorcet winner, but not a:

\begin{tabular}{|c|c|c|c|c|c|c|c|}
\hline 3 & 3 & 4 & & & $a$ & $b$ & $c$ \\
\hline$a$ & $b$ & $c$ & $a$ & $a$ & - & 9 & 5 \\
\hline$b$ & $c$ & $a$ & $c$ & $b$ & 3 & - & 6 \\
\hline$c$ & $a$ & $b$ & $b$ & $c$ & 7 & 6 & - \\
\hline
\end{tabular}


Now CC requires $c$ to be chosen uniquely, and this violates TP since a is no longer chosen.

This cannot happen if $b$ is chosen additionally to $c$, since adding rankings with $b$ on top will imply that beeps on beating $c$ by majority, so that c cannot become a Condorcet winner. Indeed, adding (e.g.) four voters with ranking bac to the original profile results in:

\begin{tabular}{|c|c|c|c|c|c|c|c|}
\hline 3 & 3 & 4 & & & $a$ & $h$ & $c$ \\
\hline$a$ & $b$ & $c$ & $b$ & $a$ & - & 7 & 7 \\
\hline$b$ & $c$ & $a$ & $w$ & $b$ & 7 & & 10 \\
\hline$c$ & $a$ & $b$ & 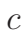 & $c$ & 7 & 4 & - \\
\hline
\end{tabular}

so that $a$ and $b$ are both Condorcet winners, and $b$ remains to be chosen.

What we learn from this example is the following. If $x$ and $y$ are alternatives and, at some profile $R^{N}$ without Condorcet winners, we have $m_{y}\left(R^{N}\right)<m_{x}\left(R^{N}\right)$, then choosing $x$ and adding rankings with $x$ on top and (as worst case) $y$ second may cause $y$ to become a Condorcet winner before $x$, unless already at $R^{N}, x$ has sufficient support against $y$ : we need that $n_{x y}\left(R^{N}\right)>-m_{y}\left(R^{N}\right)$, since then $y$ can never become a Condorcet winner by adding rankings with $x$ on top. This condition is equivalent to the condition $n_{y x}\left(R^{N}\right)<m_{y}\left(R^{N}\right)$. Indeed, one can easily check that in Example 4.1 the inequality holds for $x=b$ and $y=c$ but not for $x=a$ and $y=c$.

These considerations lead to the following definition.

Definition 4.1. The voting correspondence $H$ is defined by

$$
H\left(R^{N}\right)=\left\{x \in A:\left[m_{y}\left(R^{N}\right)<m_{x}\left(R^{N}\right) \Rightarrow n_{y x}\left(R^{N}\right)<m_{y}\left(R^{N}\right)\right] \text { for all } y \in A\right\}
$$

for every profile $R^{N}$.

Clearly, the implication in the definition of $H$ is trivially satisfied for alternatives $x$ against which resistance $m_{x}\left(R^{N}\right)$ is minimal, hence $M\left(R^{N}\right) \subseteq H\left(R^{N}\right)$.

Remark 4.2. In Pérez (2001) in Lemma 1, which uses a reformulation of Claim 3 of Moulin (1988), a related condition is used to identify alternatives that should not be chosen by a voting correspondence satisfying TP and a weaker form of CC. In contrast, we provide a condition to determine alternatives that could be chosen by a voting correspondence that satisfies CC and TP.

The main result of this chapter will be that $H$ is the maximal correspondence satisfying $\mathrm{CC}, \mathrm{TP}$ and BP and that there is no strict subcorrespondence of $H$ that contains all voting correspondences with these three properties, which are unequal to $H$. Let us first prove the following result. 
Proposition 4.2. H satisfies $C C, T P$ and $B P$.

Proof. First, we show that $H$ satisfies CC. Let $R^{N} \in L^{N}$ such that $\mathcal{C}\left(R^{N}\right) \neq \emptyset$. Then $\mathcal{C}\left(R^{N}\right)=M\left(R^{N}\right) \subseteq H\left(R^{N}\right)$. Suppose that $x \in A \backslash \mathcal{C}\left(R^{N}\right)$. Take $y \in \mathcal{C}\left(R^{N}\right)$. Then $m_{y}\left(R^{N}\right) \leq 0<m_{x}\left(R^{N}\right)$. Hence $n_{x y}\left(R^{N}\right) \leq 0$, which is equivalent to $n_{y x}\left(R^{N}\right) \geq 0$. Thus, $n_{y x}\left(R^{N}\right) \geq m_{y}\left(R^{N}\right)$, so that $x \notin H\left(R^{N}\right)$.

Next we show that $H$ satisfies TP. Let $R^{N}$ be a profile and $x \in A$ with $x \in H\left(R^{N}\right)$. Let $R^{\prime} \in L$ with $t\left(R^{\prime}\right)=x$. First, if $\mathcal{C}\left(R^{N}\right) \neq \emptyset$, then $H\left(R^{N}\right)=\mathcal{C}\left(R^{N}\right)=M\left(R^{N}\right)$ by the first part of the proof. Then, by Proposition 4.1, $x \in M\left(R^{N}, R^{\prime}\right)$, hence $x \in$ $H\left(R^{N}, R^{\prime}\right)$ and TP is satisfied. Second, if $\mathcal{C}\left(R^{N}\right)=\emptyset$, then suppose there is $y \in A$ with $m_{y}\left(R^{N}, R^{\prime}\right)<m_{x}\left(R^{N}, R^{\prime}\right)$. We have to show that $n_{y x}\left(R^{N}, R^{\prime}\right)<m_{y}\left(R^{N}, R^{\prime}\right)$. If $m_{y}\left(R^{N}\right)<m_{x}\left(R^{N}\right)$ then $n_{y x}\left(R^{N}, R^{\prime}\right)=n_{y x}\left(R^{N}\right)-1<m_{y}\left(R^{N}\right)-1 \leq m_{y}\left(R^{N}, R^{\prime}\right)$, where the strict inequality follows since $x \in H\left(R^{N}\right)$. If $m_{y}\left(R^{N}\right) \geq m_{x}\left(R^{N}\right)$ then $m_{x}\left(R^{N}, R^{\prime}\right)>m_{y}\left(R^{N}, R^{\prime}\right) \geq m_{y}\left(R^{N}\right)-1 \geq m_{x}\left(R^{N}\right)-1=m_{x}\left(R^{N}, R^{\prime}\right)$, a contradiction, so this case cannot occur.

Lastly, we show that $H$ satisfies BP. Let $R^{N} \in L^{N}, R^{\prime} \in L$ with $b\left(R^{\prime}\right)=x$, and suppose that $x \in H\left(R^{N}, R^{\prime}\right)$. We show that $x \in H\left(R^{N}\right)$. Suppose $m_{y}\left(R^{N}\right)<m_{x}\left(R^{N}\right)$ for some $y \in A$. Then $m_{y}\left(R^{N}, R^{\prime}\right)<m_{x}\left(R^{N}, R^{\prime}\right)$. Since $x \in H\left(R^{N}, R^{\prime}\right)$ this implies $n_{y x}\left(R^{N}, R^{\prime}\right)<m_{y}\left(R^{N}, R^{\prime}\right)$, hence $n_{y x}\left(R^{N}\right)+1<m_{y}\left(R^{N}, R^{\prime}\right)$. This implies $n_{y x}\left(R^{N}\right)<m_{y}\left(R^{N}, R^{\prime}\right)-1 \leq m_{y}\left(R^{N}\right)+1-1=m_{y}\left(R^{N}\right)$. Hence, $x \in H\left(R^{N}\right)$.

Now we show that $H$ not only satisfies CC and TP but is, moreover, the maximal voting correspondence satisfying these conditions, in the following sense.

Proposition 4.3. Let $C$ be a voting correspondence satisfying CC and TP. Then $C\left(R^{N}\right) \subseteq$ $H\left(R^{N}\right)$ for every $R^{N} \in L^{N}$.

Proof. Let $R^{N} \in L^{N}$ and suppose that for some $x \in A$ we have $x \in C\left(R^{N}\right) \backslash H\left(R^{N}\right)$. We derive a contradiction. Since $x \notin H\left(R^{N}\right)$, there is a $y \in A$ such that $m_{y}\left(R^{N}\right)<$ $m_{x}\left(R^{N}\right)$ and $n_{y x}\left(R^{N}\right) \geq m_{y}\left(R^{N}\right)$. By CC of $C$ and $H$ we must have $m_{y}\left(R^{N}\right)>0$. Hence, $n_{y x}\left(R^{N}\right)>0$. Equivalently, $n_{x y}\left(R^{N}\right)<0$, which implies that $m_{y}\left(R^{N}\right)=$ $n_{\widetilde{x} y}\left(R^{N}\right)$ for some $\widetilde{x} \in A \backslash\{x\}$, and in particular, $x$ does not have maximal resistance against $y$ at the profile $R^{N}$. Let $R^{\prime} \in L$ such that $x R^{\prime} y R^{\prime} z$ for all $z \in A \backslash\{x, y\}$. Then $m_{x}\left(R^{N}, R^{\prime}\right)=m_{x}\left(R^{N}\right)-1$ and $m_{y}\left(R^{N}, R^{\prime}\right)=m_{y}\left(R^{N}\right)-1$. Thus, we still have $m_{y}\left(R^{N}, R^{\prime}\right)<m_{x}\left(R^{N}, R^{\prime}\right)$, and $n_{y x}\left(R^{N}, R^{\prime}\right)=n_{y x}\left(R^{N}\right)-1 \geq m_{y}\left(R^{N}\right)-1=$ $m_{y}\left(R^{N}, R^{\prime}\right)$. Therefore, if we keep on adding the ranking $R^{\prime}$, then $n_{y x}\left(R^{N}, R^{\prime}, \ldots, R^{\prime}\right)$ decreases but still $n_{y x}\left(R^{N}, R^{\prime}, \ldots, R^{\prime}\right) \geq m_{y}\left(R^{N}, R^{\prime}, \ldots, R^{\prime}\right)$. Hence, after adding $m_{y}\left(R^{N}\right)$ rankings $R^{\prime}$ we have $m_{y}\left(R^{N}, R^{\prime}, \ldots, R^{\prime}\right)=0$ while $m_{x}\left(R^{N}, R^{\prime}, \ldots, R^{\prime}\right)>$ $m_{y}\left(R^{N}, R^{\prime}, \ldots, R^{\prime}\right)$. Then by CC, $y \in C\left(R^{N}, R^{\prime}, \ldots, R^{\prime}\right)$ and $x \notin C\left(R^{N}, R^{\prime}, \ldots, R^{\prime}\right)$. This violates TP of $C$. 
Combining Propositions 4.2 and 4.3 we obtain that $H$ is the maximal voting correspondence that is Condorcet Consistent and avoids the two no show paradoxes, i.e., it satisfies properties BP and $\mathrm{TP}$, in the sense that it contains every other voting correspondence with these three properties. Hence, a necessary condition for a voting correspondence to satisfy the three properties is that it must be a subcorrespondence of $H$. By using the following lemma we can even strengthen this result. For every $a \in A$, define the voting correspondence $C_{a}$ by

$$
C_{a}\left(R^{N}\right)=\left\{\begin{array}{cc}
M\left(R^{N}\right) & \text { if } a \notin H\left(R^{N}\right) \\
M\left(R^{N}\right) \cup\{a\} & \text { if } a \in H\left(R^{N}\right)
\end{array}\right.
$$

for every $R^{N} \in L^{N}$.

Lemma 4.1. For every $a \in A, C_{a}$ satisfies $C C, T P$, and $B P$.

Proof. Let $a \in A$ and $R^{N} \in L^{N}$.

If $\mathcal{C}\left(R^{N}\right) \neq \emptyset$ then $H\left(R^{N}\right)=M\left(R^{N}\right)=\mathcal{C}\left(R^{N}\right)$ by Propositions 4.1 and 4.2 , hence $C_{a}\left(R^{N}\right)=\mathcal{C}\left(R^{N}\right)$. This shows CC of $C_{a}$.

Let $x \in C_{a}\left(R^{N}\right)$ and $R^{\prime} \in L$ with $t\left(R^{\prime}\right)=x$. If $x \in M\left(R^{N}\right)$ then $x \in M\left(R^{N}, R^{\prime}\right)$ by Proposition 4.1, hence $x \in C_{a}\left(R^{N}, R^{\prime}\right)$. If $a \in H\left(R^{N}\right)$ and $x=a$, then $x=a \in$ $H\left(R^{N}, R^{\prime}\right)$ by Proposition 4.2, hence $x \in C_{a}\left(R^{N}, R^{\prime}\right)$. Hence, $C_{a}$ satisfies TP.

Finally, suppose $y \in A$ and $y \notin C_{a}\left(R^{N}\right)$, and let $\widetilde{R} \in L$ with $y=b(\widetilde{R})$. Since $y \notin$ $M\left(R^{N}\right)$, we have that $y \notin M\left(R^{N}, \widetilde{R}\right)$ by Proposition 4.1. If $y \neq a$ then $y \notin C_{a}\left(R^{N}, R^{\prime}\right)$. If $y=a$ then $a \notin H\left(R^{N}\right)$ and therefore $y=a \notin H\left(R^{N}, \widetilde{R}\right)$ by Proposition 4.2, so that again $y \notin C_{a}\left(R^{N}, R^{\prime}\right)$. Hence, $C_{a}$ satisfies BP.

For voting correspondences $C$ and $C^{\prime}$ we write $C \subseteq C^{\prime}$ if $C\left(R^{N}\right) \subseteq C^{\prime}\left(R^{N}\right)$ for every profile $R^{N}$ and $C \varsubsetneqq C^{\prime}$ if $C \subseteq C^{\prime}$ and there is a profile $R^{N}$ for which $C\left(R^{N}\right) \varsubsetneqq C^{\prime}\left(R^{N}\right)$.

We can now state our main result.

Theorem 4.1. $C \subseteq H$ for all voting correspondences $C$ satisfying $C C, T P$, and BP. Moreover, there is no voting correspondence $H^{\prime} \varsubsetneqq H$ such that $C \subseteq H^{\prime}$ for all voting correspondences $C \neq H$ that satisfy $C C, T P$, and $B P$.

Proof. The first statement follows from Propositions 4.1 and 4.2. For the second statement, let $H^{\prime} \varsubsetneqq H$ be a voting correspondence and take $R^{N} \in L^{N}$ and $a \in H\left(R^{N}\right) \backslash$ $H^{\prime}\left(R^{N}\right)$. By Lemma 4.1, $C_{a}$ satisfies CC, TP, and BP, however $C_{a} \not H^{\prime}$.

Hence, $H$ is the union of all voting correspondences $C \neq H$ satisfying CC, TP, and BP. Thus, there is no strict subcorrespondence of $H$ that contains all voting correspondences with these three properties, which are unequal to $H$. 
From Theorem 4.1 we can drop BP but not TP. In other words, there are voting correspondences satisfying $\mathrm{CC}$ and $\mathrm{BP}$ which are not subcorrespondences of $H$. An example is the following.

Example 4.2. For a profile $R^{N} \in L^{N}$ and an alternative $x \in A$ let $\eta_{x}$ denote the minimum number of voters that have to be removed from the profile in order to make $x$ a Condorcet winner, hence

$$
\eta_{x}\left(R^{N}\right)=\min \left\{\left|N^{\prime}\right|: N^{\prime} \varsubsetneqq N \text { and } x \in \mathcal{C}\left(R^{N \backslash N^{\prime}}\right)\right\} .
$$

Define the voting correspondence $Y$ (cf. Young, 1977) by

$$
Y\left(R^{N}\right)=\left\{x \in A: \eta_{x}\left(R^{N}\right) \leq \eta_{y}\left(R^{N}\right) \text { for all } y \in A\right\}
$$

for all $R^{N} \in L^{N}$. Clearly, since for each $x \in A$ we have $\eta_{x}\left(R^{N}\right)=0$ if and only if $x \in \mathcal{C}\left(R^{N}\right), Y$ is Condorcet Consistent. To show that $Y$ satisfies BP, suppose that $R^{N} \in L^{N}$ and $x \in A$ such that $x \notin Y\left(R^{N}\right)$. Let $y \in A$ with $\eta_{y}\left(R^{N}\right)<\eta_{x}\left(R^{N}\right)$, and let $R^{\prime} \in L$ with $b\left(R^{\prime}\right)=x$. Then $\eta_{x}\left(R^{N}, R^{\prime}\right)=\eta_{x}\left(R^{N}\right)+1$, and therefore $\eta_{y}\left(R^{N}, R^{\prime}\right) \leq$ $\eta_{y}\left(R^{N}\right)+1<\eta_{x}\left(R^{N}\right)+1=\eta_{x}\left(R^{N}, R^{\prime}\right)$. Hence, $x \notin Y\left(R^{N}, R^{\prime}\right)$.

In order to show that $Y$ is not a subcorrespondence of $H$, we borrow the following profile $R^{N}$ with 39 voters and 5 alternatives from Felsenthal \& Nurmi (2016):

\begin{tabular}{cccccccc}
11 & 10 & 10 & 2 & 2 & 2 & 1 & 1 \\
\hline$b$ & $e$ & $a$ & $e$ & $e$ & $c$ & $d$ & $a$ \\
$a$ & $c$ & $c$ & $c$ & $d$ & $b$ & $c$ & $b$ \\
$d$ & $b$ & $d$ & $d$ & $c$ & $a$ & $b$ & $d$ \\
$e$ & $d$ & $b$ & $b$ & $b$ & $d$ & $a$ & $e$ \\
$c$ & $a$ & $e$ & $a$ & $a$ & $e$ & $e$ & $c$
\end{tabular}

It is not hard to show that $\eta_{a}\left(R^{N}\right)=17, \eta_{b}\left(R^{N}\right)=15, \eta_{c}\left(R^{N}\right)=13, \eta_{d}\left(R^{N}\right)=16$, and $\eta_{e}\left(R^{N}\right)=11$, so that $Y\left(R^{N}\right)=\{e\} .{ }^{l}$ However, $m_{d}\left(R^{N}\right)=9<m_{e}\left(R^{N}\right)=11$ but $n_{d e}\left(R^{N}\right)=11 \nless m_{d}\left(R^{N}\right)$, so that $e \notin H\left(R^{N}\right)$. By Proposition 4.3 this implies that $Y$ does not satisfy $T P^{2}$

\footnotetext{
${ }^{1}$ For $e$ to become a Condorcet winner one must remove for example the ten preferences with $a$ on top and one of the preferences with $c$ on top.

${ }^{2}$ Pérez (2001) and Felsenthal \& Nurmi (2016) also show that $Y$ does not satisfy TP.
} 
It is easy to see, that Young's rule is not the maximal voting correspondence satisfying $\mathrm{BP}$ and CC. As we show below, this is the voting correspondence $\widehat{B}$ defined recursively by

$$
\widehat{B}\left(R^{N}\right)= \begin{cases}\mathcal{C}\left(R^{N}\right) & \text { if } \mathcal{C}\left(R^{N}\right) \neq \emptyset \\ \left\{x \in A: \forall i \in N\left[b\left(R^{i}\right)=x \Rightarrow x \in \widehat{B}\left(R^{N \backslash\{i\}}\right)\right]\right\} & \text { if } \mathcal{C}\left(R^{N}\right)=\emptyset\end{cases}
$$

for all $R^{N} \in L^{N}$. Observe that, if $\mathcal{C}\left(R^{N}\right)=\emptyset$, then $\widehat{B}\left(R^{N}\right)$ contains in particular all alternatives that do not occur at bottom in $R^{N}$.

Proposition 4.4. The voting correspondence $\widehat{B}$ satisfies $C C$ and BP. Moreover, if a voting correspondence $C$ satisfies $C C$ and $B P$, then $C \subseteq \widehat{B}$.

Proof. That $\widehat{B}$ satisfies CC and BP is straightforward. Let $C$ satisfy CC and BP. Then $C\left(R^{N}\right)=\mathcal{C}\left(R^{N}\right)=\widehat{B}\left(R^{N}\right)$ whenever $R^{N} \in L^{N}$ and $|N| \in\{1,2\}$. Now let $n \geq 3$ and assume that $C\left(R^{N}\right) \subseteq \widehat{B}\left(R^{N}\right)$ whenever $R^{N} \in L^{N}$ with $|N|<n$. Suppose there is $x \in C\left(R^{N}\right) \backslash \widehat{B}\left(R^{N}\right)$ for some $R^{N} \in L^{N},|N|=n$. Then there is $i \in N$ with $x=b\left(R^{i}\right)$ and $x \notin \widehat{B}\left(R^{N \backslash\{i\}}\right)$, hence $x \notin C\left(R^{N \backslash\{i\}}\right)$. This violates BP of $C$.

\subsection{CONDORCET CONSISTENCY, TOP PROPERTY, AND BOTTOM PROPERTY FOR VOTING FUNCTIONS}

A voting function $F$ is a single-valued voting correspondence. The definitions of the Top Property and the Bottom Property therefore follow from those for a voting correspondence. The adapted definition of Condorcet Consistency is as follows.

Condorcet Consistency (CC) For every profile $R^{N}$ such that $\mathcal{C}\left(R^{N}\right) \neq \emptyset, F\left(R^{N}\right) \in$ $\mathcal{C}\left(R^{N}\right)$.

This definition reflects the fact that (weak) Condorcet winners are not necessarily unique and there is no obvious way a priori to distinguish between them.

We start with the following result, which still needs a proof, due to the adapted definition of CC. This proof, however, is a minor modification of the proof of Proposition 4.3, and for completeness provided in the Appendix.

Proposition 4.5. Let voting function $F$ satisfy $C C$ and TP. Then $F$ is a selection from $H$.

Thus, for a voting function to be Condorcet Consistent and satisfy the Top Property, it has to select from $H$, and the question arises how this can be done. It cannot be done arbitrarily, as the following example shows. 
Example 4.3. Consider the ten voter profile $R^{N}$ given by

\begin{tabular}{lll}
3 & 3 & 4 \\
\hline$a$ & $b$ & $c$ \\
$b$ & $c$ & $a$ \\
$c$ & $a$ & $b$
\end{tabular}

of Example 4.1. Then $H\left(R^{N}\right)=\{b, c\}$. Suppose that $F\left(R^{N}\right)=b$. Adding a voter with ranking $R^{\prime}=$ bac results in the following 11 -voter profile $\left(R^{N}, R^{\prime}\right)$ :

\begin{tabular}{cccc}
3 & 3 & 4 & 1 \\
\hline$a$ & $b$ & $c$ & $b$ \\
$b$ & $c$ & $a$ & $a$ \\
$c$ & $a$ & $b$ & $c$
\end{tabular}

Now $m_{a}\left(R^{N}, R^{\prime}\right)=m_{b}\left(R^{N}, R^{\prime}\right)=m_{c}\left(R^{N}, R^{\prime}\right)=3$, so that $H\left(R^{N}, R^{\prime}\right)=\{a, b, c\}$. However, if we wish $F$ to satisfy $T P$, we must have $F\left(R^{N}, R^{\prime}\right)=b$.

A general characterization of all selections from $H$ that satisfy $\mathrm{CC}$ and $\mathrm{TP}$ and/or BP is yet out of reach. However, we will exhibit some possibilities and impossibilities in the remainder of this section.

\subsubsection{Selecting independently}

We discuss selections from $H$ that are independent of the underlying rankings and only depend on the alternatives chosen by $H$. More precisely, we consider the following property for a selection $F$ from a correspondence $C$.

Independence of Irrelevant Alternatives (IIA) Let $C$ be a voting correspondence and let $F$ be a selection from it. For all profiles $R^{N} \in L^{N}$ and $\widetilde{R}^{M} \in L^{M}$, if $C\left(Q^{M}\right) \subseteq$ $C\left(R^{N}\right)$ and $F\left(R^{N}\right) \in C\left(\widetilde{R}^{M}\right)$, then $F\left(\widetilde{R}^{M}\right)=F\left(R^{N}\right)$.

If this condition is satisfied, then we say that $F$ satisfies IIA with respect to $C .^{3}$

Call a voting correspondence $C$ surjective if for every $\emptyset \neq B \subseteq A$ there is a profile $R^{N}$ such that $C\left(R^{N}\right)=B$. We now have the following characterization of IIA-see the Appendix for the proof. ${ }^{4}$

\footnotetext{
${ }^{3}$ IIA is the name usually employed for Assumption 7 in Nash (1950a).

${ }^{4}$ IIA is equivalent to the Weak Axiom of Revealed Preference, and Proposition 4.6 is a familiar result stating that $F$ can be rationalized by a linear ordering.
} 
Proposition 4.6. Let voting correspondence $C$ be surjective and let voting function $F$ be a selection from $C$. Then $F$ satisfies IIA with respect to $C$ if and only if there is a $Q \in L$ such that $F\left(R^{N}\right) Q x$ for all $x \in C\left(R^{N}\right)$.

In other words, IIA is equivalent to choosing according to a fixed ranking $Q$ if the voting correspondence is surjective. The latter is true for any voting correspondence that is Condorcet Consistent:

Lemma 4.2. Let voting correspondence $C$ satisfy $C C$. Then $C$ is surjective.

The proof is given in the Appendix. Our first result about voting functions satisfying $\mathrm{CC}, \mathrm{TP}$ and $\mathrm{BP}$ is as follows.

Proposition 4.7. Every selection $F$ from $M$ that satisfies IIA with respect to $M$, satisfies $T P$ and $B P$.

Proof. Let $F$ be a selection from $M$ satisfying IIA with respect to $M$.

First, we show that $F$ satisfies TP. Let $R^{N} \in L^{N}, x=F\left(R^{N}\right)$, and $R^{\prime} \in L$ with $t\left(R^{\prime}\right)=x$. By IIA of $F$ with respect to $M$, it is sufficient to show that $M\left(R^{N}, R^{\prime}\right) \subseteq$ $M\left(R^{N}\right)$ and $x \in M\left(R^{N}, R^{\prime}\right)$. Let $y \in A$ with $y \notin M\left(R^{N}\right)$. Then $m_{y}\left(R^{N}\right)>m_{x}\left(R^{N}\right)$. Hence $m_{y}\left(R^{N}, R^{\prime}\right) \geq m_{y}\left(R^{N}\right)-1>m_{x}\left(R^{N}\right)-1=m_{x}\left(R^{N}, R^{\prime}\right)$, so that $y \notin$ $M\left(R^{N}, R^{\prime}\right)$. Thus, $M\left(R^{N}, R^{\prime}\right) \subseteq M\left(R^{N}\right)$. Further, for every $z \in A, m_{x}\left(R^{N}, R^{\prime}\right)=$ $m_{x}\left(R^{N}\right)-1 \leq m_{z}\left(R^{N}\right)-1 \leq m_{z}\left(R^{N}, R^{\prime}\right)$, so that $x \in M\left(R^{N}, R^{\prime}\right)$.

Second, we show that $F$ satisfies BP. Suppose that $R^{N} \in L^{N}$ and $x \in A$ and $F\left(R^{N}\right) \neq$ $x$. Then $M\left(R^{N}\right) \backslash\{x\} \neq \emptyset$. Let $R^{\prime} \in L$ such that $b\left(R^{\prime}\right)=x$. We show that $F\left(R^{N}, R^{\prime}\right) \neq$ $x$. For all $y \in M\left(R^{N}\right) \backslash\{x\}$ we have

$$
m_{x}\left(R^{N}, R^{\prime}\right)=m_{x}\left(R^{N}\right)+1 \geq m_{y}\left(R^{N}\right)+1 \geq m_{y}\left(R^{N}, R^{\prime}\right) .
$$

If $x \notin M\left(R^{N}\right)$, then the first inequality in (8) is strict, hence $x \notin M\left(R^{N}, R^{\prime}\right)$, and in that case $F\left(R^{N}, R^{\prime}\right) \neq x$. If $x \in M\left(R^{N}\right) \cap M\left(R^{N}, R^{\prime}\right)$ then all inequalities in (8) are equalities, implying $M\left(R^{N}\right) \subseteq M\left(R^{N}, R^{\prime}\right)$, so that $F\left(R^{N}, R^{\prime}\right) \neq x$ by IIA.

Thus, selections from the Minimax Rule according to a fixed ranking (cf. Lemma 4.2 and Proposition 4.6) prevent both no show paradoxes considered in this chapter. It turns out, however, that —if there are at least three alternatives — we cannot select independently from $H$ and still have TP or BP.

Proposition 4.8. Let $|A| \geq 3$ and let voting function $F$ be a selection from $H$ that satisfies IIA with respect to $H$. Then F neither satisfies TP nor BP.

Proof. By Lemma 4.2 and Proposition 4.6 there is a ranking $Q \in L$ such that $F\left(R^{N}\right) Q x$ for all $x \in H\left(R^{N}\right)$, for every profile $R^{N}$. Let without loss of generality $Q=a b c \ldots$ for 
some $a, b, c \in A$ and consider the following ten voter profile $R^{N}$, similar to the one in Example 4.1:

\begin{tabular}{ccc}
3 & 3 & 4 \\
\hline$a$ & $b$ & $c$ \\
$b$ & $c$ & $a$ \\
$c$ & $a$ & $b$ \\
$\vdots$ & $\vdots$ & $\vdots$
\end{tabular}

Then $H\left(R^{N}\right)=\{b, c\}$. Adding a voter with ranking $R^{\prime}=b a c \ldots$ results in the profile $\left(R^{N}, R^{\prime}\right)$ given by

\begin{tabular}{cccc}
3 & 3 & 4 & 1 \\
\hline$a$ & $b$ & $c$ & $b$ \\
$b$ & $c$ & $a$ & $a$ \\
$c$ & $a$ & $b$ & $c$ \\
$\vdots$ & $\vdots$ & $\vdots$ & $\vdots$
\end{tabular}

with $H\left(R^{N}, R^{\prime}\right)=\{a, b, c\}$ and thus $F\left(R^{N}, R^{\prime}\right)=a$, violating TP.

To exhibit a violation of BP, consider the 10 -voter profile $\widetilde{R}^{N}$ given by

\begin{tabular}{ccc}
3 & 3 & 4 \\
\hline$c$ & $a$ & $b$ \\
$a$ & $b$ & $c$ \\
$b$ & $c$ & $a$ \\
$\vdots$ & $\vdots$ & $\vdots$
\end{tabular}

so that $H\left(\widetilde{R}^{N}\right)=\{a, b\}$ and $F\left(\widetilde{R}^{N}\right)=a$. Adding a voter with ranking $\check{R}=c a \ldots b$ results in the profile $\left(\widetilde{R}^{N}, \check{R}\right)$ given by

\begin{tabular}{cccc}
3 & 3 & 4 & 1 \\
\hline$c$ & $a$ & $b$ & $c$ \\
$a$ & $b$ & $c$ & $a$ \\
$b$ & $c$ & $a$ & $\vdots$ \\
$\vdots$ & $\vdots$ & $\vdots$ & $b$
\end{tabular}

so that $H\left(\widetilde{R}^{N}, \check{R}\right)=\{b, c\}$ and $F\left(\widetilde{R}^{N}, \check{R}\right)=b$, a violation of BP since $b=b(\check{R})$.

In the next subsection we provide some positive results with respect to selections from $H$. 


\subsubsection{Non-independent selections}

According to Proposition 4.7, independent (IIA) selections from $M$ satisfy TP and BP. We now provide examples of selections from $M$ that satisfy TP but not BP, and conversely. Consequently, those selections cannot be achieved by maximizing according to a fixed ranking. (See the Appendix for the proofs of the claims in this subsection.)

Example 4.4. Define the voting correspondence $\bar{M}$ by

$$
\begin{aligned}
\bar{M}\left(R^{N}\right)= & \left\{x \in M\left(R^{N}\right):\left|\left\{i \in N: b\left(R^{i}\right)=x\right\}\right|\right. \\
& \left.\leq\left|\left\{i \in N: b\left(R^{i}\right)=y\right\}\right| \text { for all } y \in M\left(R^{N}\right)\right\}
\end{aligned}
$$

for every profile $R^{N}$. Hence $\bar{M}\left(R^{N}\right)$ contains those alternatives from $M\left(R^{N}\right)$ that are ranked last by a minimal number of voters. We fix a ranking $Q \in L$ and define the selection $\bar{F}$ from $\bar{M}$ such that, for every profile $R^{N}, \bar{F}\left(R^{N}\right) Q y$ for all $y \in \bar{M}\left(R^{N}\right)$ if $|N|$ is even, and $y Q \bar{F}\left(R^{N}\right)$ for all $y \in \bar{M}\left(R^{N}\right)$ if $|N|$ is odd. Then $\bar{F}$ satisfies BP but not TP.

Example 4.5. Define the voting correspondence $\check{M}$ by

$$
\begin{aligned}
\check{M}\left(R^{N}\right)= & \left\{x \in M\left(R^{N}\right):\left|\left\{i \in N: b\left(R^{i}\right)=x\right\}\right|\right. \\
& \left.\geq\left|\left\{i \in N: b\left(R^{i}\right)=y\right\}\right| \text { for all } y \in M\left(R^{N}\right)\right\}
\end{aligned}
$$

for every profile $R^{N}$. Fix a ranking $Q \in L$ and define the selection $\breve{F}$ from $\check{M}$ such that, for every profile $R^{N}, \breve{F}\left(R^{N}\right) Q$ y for all $y \in \check{M}\left(R^{N}\right)$. Then $\breve{F}$ satisfies TP but not BP.

Proposition 4.8 states that independent selections from $H$ cannot satisfy TP or BP. Nevertheless, there exist selections from $H$ that are not selections from $M$ and that satisfy TP and BP; TP but not BP; or BP but not TP. The following examples show this. (The proofs are in the Appendix.)

Example 4.6. Let $A=\{a, b, c\}$, consider again the first profile given in Example 4.1:

\begin{tabular}{ccc}
3 & 3 & 4 \\
\hline$a$ & $b$ & $c$ \\
$b$ & $c$ & $a$ \\
$c$ & $a$ & $b$
\end{tabular}

and call this profile $R^{*}$. Let $Q=$ bac $\in L$ and for every profile $R^{N} \neq R^{*}$ define $F\left(R^{N}\right) \in$ $M\left(R^{N}\right)$ such that $F\left(R^{N}\right) Q x$ for all $x \in M\left(R^{N}\right) \backslash\left\{F\left(R^{N}\right)\right\}$; and let $F\left(R^{*}\right)=b \in$ $H\left(R^{*}\right)=\{b, c\}$. Note that $b \notin\{c\}=M\left(R^{*}\right)$. This voting function $F$ satisfies both $T P$ and $B P$. 
Example 4.7. Let $A=\{a, b, c\}$, consider again the profile $R^{*}$ of Example 4.6, let now $Q=b c a \in L$, and define $F$ as in Example 4.6, now using this $Q$. Then F satisfies TP but not $B P$.

Example 4.8. Let $A=\{a, b, c\}$, consider again the profile $R^{*}$ of Example 4.6, and let now $Q=c a b \in L$. Define $\bar{F}\left(R^{N}\right)$ as in Example 4.4 for every profile $R^{N} \neq R^{*}$, and let $F\left(R^{*}\right)=b$. Then $\bar{F}$ satisfies BP but not TP.

\subsection{WEAK CONDORCET CONSISTENCY}

In order to further relate this chapter with the existing literature-in particular with Moulin (1988) and Pérez (2001) — we now consider a weaker notion of Condorcet Consistency. That is, we require the voting correspondence to pick the unique strong Condorcet winner if there is one, but do not require anything if there are only weak Condorcet winners. Again, we find the maximal voting correspondence satisfying TP, $\mathrm{BP}$ and this weaker notion of Condorcet Consistency. As can be expected, this voting correspondence is larger than $H$.

For $R^{N} \in L^{N}$ denote the possibly empty set containing the strong Condorcet winner by $\mathcal{C}_{s}\left(R^{N}\right)$, hence

$$
\mathcal{C}_{s}\left(R^{N}\right)=\left\{x \in A: m_{x}\left(R^{N}\right)<0\right\}
$$

Weak Condorcet Consistency (WCC) Let $C$ be a voting correspondence. For every profile $R^{N}$ such that $\mathcal{C}_{s}\left(R^{N}\right) \neq \emptyset, C\left(R^{N}\right)=\mathcal{C}_{s}\left(R^{N}\right)$.

It is obvious that $H$ as well as $M$ satisfy WCC. However, Proposition 4.3 and Theorem 4.1 no longer hold. A larger voting correspondence turns out to be the maximal one satisfying TP and WCC.

Definition 4.2. The voting correspondence $\widetilde{H}$ is defined by

$$
\widetilde{H}\left(R^{N}\right)=\left\{x \in A:\left[m_{y}\left(R^{N}\right)<m_{x}\left(R^{N}\right) \Rightarrow n_{y x}\left(R^{N}\right) \leq m_{y}\left(R^{N}\right)\right] \text { for all } y \in A\right\}
$$

for every profile $R^{N}$.

The difference with $H$ is that the weak inequality in Definition 4.2 is strict in the definition of $H$.

The proofs of the next two propositions are quite similar to the proofs of the corresponding results for $H$ in Section 4.3. For completeness they are given in the Appendix.

Proposition 4.9. $\widetilde{H}$ satisfies $W C C, T P$ and $B P$.

As $H \varsubsetneqq \widetilde{H}$, it follows together with Proposition 4.2 that $\widetilde{H}$ does not satisfy CC. 
Proposition 4.10. Let $C$ be a voting correspondence satisfying WCC and TP. Then $C\left(R^{N}\right) \subseteq \widetilde{H}\left(R^{N}\right)$ for every $R^{N} \in L^{N}$.

Following from the last two propositions we obtain that $\widetilde{H}$ is the maximal correspondence satisfying WCC, TP, and BP.

Also Lemma 4.1 can be easily adapted. We derive the following Theorem.

Theorem 4.2. $C \subseteq \widetilde{H}$ for all voting correspondences $C$ satisfying $C C$, $T P$, and $B P$. Moreover, there is no voting correspondence $H^{\prime} \varsubsetneqq \widetilde{H}$ such that $C \subseteq H^{\prime}$ for all voting correspondences $C \neq \widetilde{H}$ that satisfy $C C, T P$, and $B P$.

If we consider voting functions however, the negative result given in Proposition 4.8 still holds.

Proposition 4.11. Let $|A| \geq 3$ and let voting function $F$ be a selection from $\widetilde{H}$ that satisfies IIA with respect to $\widetilde{H}$. Then $F$ does not satisfy TP and F does not satisfy BP.

This proposition immediately follows from $\widetilde{H}\left(R^{N}\right)=H\left(R^{N}\right)$ and $\widetilde{H}\left(R^{N}, R^{\prime}\right)=$ $H\left(R^{N}, R^{\prime}\right)$ for $R^{N}$ and $R^{\prime}$ as given in the first example in the proof of Proposition 4.8. Also $\widetilde{H}\left(\widetilde{R}^{N}\right)=\widetilde{H}\left(\widetilde{R}^{N}\right)$ and $\widetilde{H}\left(\widetilde{R}^{N}, \check{R}\right)=H\left(\widetilde{R}^{N}, \check{R}\right)$ for $\widetilde{R}^{N}$ and $\check{R}$ as given in the second example in the proof of Proposition 4.8. The examples given in Section 4.4.2 also show that there are selections from $\widetilde{H}$ that satisfy WCC, TP and BP.

\subsection{CONCLUDING REMARKS}

We have provided a necessary condition for a voting correspondence to satisfy [weak] Condorcet Consistency while at the same time ruling out the two strong no show paradoxes, namely that it should be a subcorrespondence of $H[\widetilde{H}]$. The basic idea leading to the definition of $H[\widetilde{H}]$ is that by adding voters Condorcet winners may pop up expelling existing winners - the condition defining $H[\widetilde{H}]$ rules out exactly this.

A remaining problem is to find sufficient conditions for voting correspondences to satisfy TP, BP and CC. Also, and related to this, the construction of all selections from $H[\widetilde{H}]$, i.e., voting functions, that choose a Condorcet winner if there is one and rule out the strong no show paradoxes, is an open problem.

\subsection{APPENDIX}

\section{Remaining proofs}

Proof of Proposition 4.1. If $\mathcal{C}\left(R^{N}\right) \neq \emptyset$ for some profile $R^{N}$, then by Remark 4.1 either there is a unique alternative $x$ with $m_{x}\left(R^{N}\right)<0$, in which case $M\left(R^{N}\right)=$ 
$\mathcal{C}\left(R^{N}\right)=\{x\}$, or $\mathcal{C}\left(R^{N}\right)=\left\{x \in A: m_{x}\left(R^{N}\right)=0\right\}$, in which case $M\left(R^{N}\right)=\mathcal{C}\left(R^{N}\right)=$ $\left\{x \in A: m_{x}\left(R^{N}\right)=0\right\}$. Hence, $M$ satisfies CC.

Next we show that $M$ satisfies TP. Let $x \in M\left(R^{N}\right)$. Then $m_{x}\left(R^{N}\right) \leq m_{y}\left(R^{N}\right)$ for all $y \in A$. Consider a ranking $R^{\prime} \in L$ such that $t\left(R^{\prime}\right)=x$. Then $m_{x}\left(R^{N}, R^{\prime}\right)=m_{x}\left(R^{N}\right)-1$ and $m_{y}\left(R^{N}, R^{\prime}\right) \geq m_{y}\left(R^{N}\right)-1$ for all $y \in A \backslash\{x\}$. Hence $m_{x}\left(R^{N}, R^{\prime}\right) \leq m_{y}\left(R^{N}, R^{\prime}\right)$ for all $y \in A$, so that $x \in M\left(R^{N}, R^{\prime}\right)$.

It remains to show that $M$ satisfies BP. Suppose $x \notin M\left(R^{N}\right)$. Choose $y \in A$ with $m_{x}\left(R^{N}\right)>m_{y}\left(R^{N}\right)$. Consider a ranking $R^{\prime} \in L$ such that $b\left(R^{\prime}\right)=x$. Then $m_{x}\left(R^{N}, R^{\prime}\right)=m_{x}\left(R^{N}\right)+1$ and $m_{y}\left(R^{N}, R^{\prime}\right) \leq m_{y}\left(R^{N}\right)+1$. Hence, $m_{x}\left(R^{N}, R^{\prime}\right)>$ $m_{y}\left(R^{N}, R^{\prime}\right)$, so that $x \notin M\left(R^{N}, R^{\prime}\right)$.

Proof of Lemma 4.2 Let $\emptyset \neq B=\left\{x_{1}, \ldots, x_{k}\right\} \subseteq A$. Let $R^{1}, R^{2} \in L$ such that $x_{1} R^{1} \ldots$ $R^{1} x_{k} R^{1} x$ and $x_{k} R^{2} \ldots R^{2} x_{1} R^{2} x$ for all $x \in A \backslash B$. Then $\mathcal{C}\left(R^{1}, R^{2}\right)=B$, so that $C\left(R^{1}, R^{2}\right)=B$ by CC of $C$.

Proof of Proposition 4.5. Let $R^{N} \in L^{N}$ and suppose that $x=F\left(R^{N}\right) \notin H\left(R^{N}\right)$. We derive a contradiction. Since $x \notin H\left(R^{N}\right)$, there is a $y \in A$ such that $m_{y}\left(R^{N}\right)<$ $m_{x}\left(R^{N}\right)$ and $n_{y x}\left(R^{N}\right) \geq m_{y}\left(R^{N}\right)$. If $m_{y}\left(R^{N}\right) \leq 0$ then by CC of $F$ and Remark 4.1 either $x=y$ or $m_{y}\left(R^{N}\right)=m_{x}\left(R^{N}\right)=0$, but each case contradicts $m_{y}\left(R^{N}\right)<$ $m_{x}\left(R^{N}\right)$. Therefore $m_{y}\left(R^{N}\right)>0$. Hence, $n_{y x}\left(R^{N}\right)>0$. Equivalently, $n_{x y}<0$, which implies that $m_{y}\left(R^{N}\right)=n_{\widetilde{x} y}\left(R^{N}\right)$ for some $\widetilde{x} \in A \backslash\{x\}$, and in particular, $x$ does not have maximal resistance against $y$ at the profile $R^{N}$. Let $R^{\prime} \in L$ such that $x R^{\prime} y R^{\prime} z$ for all $z \in A \backslash\{x, y\}$. Then $m_{x}\left(R^{N}, R^{\prime}\right)=m_{x}\left(R^{N}\right)-1$ and $m_{y}\left(R^{N}, R^{\prime}\right)=$ $m_{y}\left(R^{N}\right)-1$. Thus, we still have $m_{y}\left(R^{N}, R^{\prime}\right)<m_{x}\left(R^{N}, R^{\prime}\right)$, and $n_{y x}\left(R^{N}, R^{\prime}\right)=$ $n_{y x}\left(R^{N}\right)-1 \geq m_{y}\left(R^{N}\right)-1=m_{y}\left(R^{N}, R^{\prime}\right)$. Therefore, if we keep on adding $R^{\prime}$, then $n_{y x}\left(R^{N}, R^{\prime}, \ldots, R^{\prime}\right)$ decreases but still $n_{y x}\left(R^{N}, R^{\prime}, \ldots, R^{\prime}\right) \geq m_{y}\left(R^{N}, R^{\prime}, \ldots, R^{\prime}\right)$. Hence, after adding $m_{y}\left(R^{N}\right)$ rankings $R^{\prime}$ we have $m_{y}\left(R^{N}, R^{\prime}, \ldots, R^{\prime}\right)=0$ while $m_{x}\left(R^{N}, R^{\prime}, \ldots, R^{\prime}\right)>m_{y}\left(R^{N}, R^{\prime}, \ldots, R^{\prime}\right)$. Hence $\mathcal{C}\left(R^{N}, R^{\prime}, \ldots, R^{\prime}\right) \neq \emptyset$ but $x \notin$ $\mathcal{C}\left(R^{N}, R^{\prime}, \ldots, R^{\prime}\right)$, so that by CC of $F, x \neq F\left(R^{N}, R^{\prime}, \ldots, R^{\prime}\right)$. This violates TP of $F$.

Proof of Proposition 4.6. Suppose that $F$ satisfies IIA with respect to $C$. We construct a linear ranking $Q$. First, take $N^{1} \subseteq \mathbb{N}$ and $R_{1}^{N^{1}} \in L^{N^{1}}$ such that $C\left(R_{1}^{N^{1}}\right)=A$. Note that $R_{1}^{N^{1}}$ exists since $C$ is surjective. Then let $F\left(R_{1}^{N^{1}}\right) Q x$ for all $x \in A \backslash\left\{F\left(R_{1}^{N^{1}}\right)\right\}$. Next, take $N^{2} \subseteq \mathbb{N}$ and $R_{2}^{N^{2}} \in L^{N^{2}}$ such that $C\left(R_{2}^{N^{2}}\right)=A \backslash\left\{F\left(R_{1}^{N^{1}}\right)\right\}$ and let $F\left(R_{2}^{N^{2}}\right) Q x$ for all $x \in A \backslash\left\{F\left(R_{1}^{N^{1}}\right), F\left(R_{2}^{N^{2}}\right)\right\}$. Continuing this way, we construct a sequence $R_{1}^{N^{1}}, \ldots, R_{|A|-1}^{N^{|A|-1}}$ and a ranking $Q \in L$. Now let $\widetilde{R}^{M}$ be an arbitrary profile and write $B=C\left(\widetilde{R}^{M}\right)$. Let $b \in B$ such that $b Q x$ for all $x \in B$. Then let $D=\{x \in A: b Q x\}$ and $R_{j}^{N^{j}}$ for some $1 \leq j \leq|A|-1$ such that $C\left(R_{j}^{N^{j}}\right)=D$ then, by construction of $Q$, $F\left(R_{j}^{N^{j}}\right)=b$. By IIA, $F\left(\widetilde{R}^{M}\right)=b$. This shows the only-if direction of the proposition. 
For the if-direction, suppose there is a ranking $Q \in L$ such that $F\left(R^{N}\right) Q x$ for every profile $R^{N}$ and every $x \in C\left(R^{N}\right)$. Suppose that $R^{N}$ and $\widetilde{R}^{M}$ are profiles with $C\left(\widetilde{R}^{M}\right) \subseteq$ $C\left(R^{N}\right)$ and $F\left(R^{N}\right) \in C\left(\widetilde{R}^{M}\right)$. Then, since $F\left(R^{N}\right) Q x$ for all $x \in C\left(R^{N}\right)$, it follows that $F\left(R^{N}\right) Q x$ for all $x \in C\left(\widetilde{R}^{M}\right)$. Hence, $F\left(R^{N}\right)=F\left(\widetilde{R}^{M}\right)$.

Proof of the claims in Example 4.4 We first show that $\bar{F}$ satisfies BP. Let $x \in A$ and $R^{N} \in L^{N}$ such that $\bar{F}\left(R^{N}\right) \neq x$. Let $R^{\prime} \in L$ with $b\left(R^{\prime}\right)=x$. We have to show that $\bar{F}\left(R^{N}, R^{\prime}\right) \neq x$. We will use the notation $\beta_{z}\left(\widetilde{R}^{P}\right)=\left|\left\{i \in P: b\left(\widetilde{R}^{i}\right)=z\right\}\right|$ for a profile $\widetilde{R}^{P}$ and an alternative $z \in A$.

If $x \notin \bar{M}\left(R^{N}, R^{\prime}\right)$ then $\bar{F}\left(R^{N}, R^{\prime}\right) \neq x$ and we are done. Thus, we assume $x \in$ $\bar{M}\left(R^{N}, R^{\prime}\right)$. This implies, in particular, that $x \in M\left(R^{N}\right)$, since otherwise $x \notin$ $M\left(R^{N}, R^{\prime}\right)$, hence $x \notin \bar{M}\left(R^{N}, R^{\prime}\right)$.

Now suppose $y \in \bar{M}\left(R^{N}\right), y \neq x$. Then $m_{y}\left(R^{N}, R^{\prime}\right) \leq m_{y}\left(R^{N}\right)+1=m_{x}\left(R^{N}\right)+$ $1=m_{x}\left(R^{N}, R^{\prime}\right)$, so that $y \in M\left(R^{N}, R^{\prime}\right)$. Also, $\beta_{y}\left(R^{N}\right) \leq \beta_{x}\left(R^{N}\right)$ implies $\beta_{y}\left(R^{N}, R^{\prime}\right)=\beta_{y}\left(R^{N}\right)<\beta_{x}\left(R^{N}\right)+1=\beta_{x}\left(R^{N}, R^{\prime}\right)$. This together with $y \in$ $M\left(R^{N}, R^{\prime}\right)$ and $x \in \bar{M}\left(R^{N}, R^{\prime}\right)$ gives a contradiction. Therefore, $\bar{M}\left(R^{N}\right)=\{x\}$, which contradicts $\bar{F}\left(R^{N}\right) \neq x$.

To show, finally, that $\bar{F}$ does not satisfy TP, we exhibit an example. Let $A=\{a, b, c\}$ and take $Q=a b c$. Consider the 6-voter profile $R^{N}$ given by

$$
\begin{array}{ccc}
2 & 2 & 2 \\
\hline a & b & c \\
b & c & a \\
c & a & b
\end{array}
$$

Then $M\left(R^{N}\right)=\bar{M}\left(R^{N}\right)=\{a, b, c\}$ and $\bar{F}\left(R^{N}\right)=a$. For the 7-voter profile $\left(R^{N}, R^{\prime}\right)$ with $R^{\prime}=a c b$ :

\begin{tabular}{cccc}
2 & 2 & 2 & 1 \\
\hline$a$ & $b$ & $c$ & $a$ \\
$b$ & $c$ & $a$ & $c$ \\
$c$ & $a$ & $b$ & $b$
\end{tabular}

we obtain $M\left(R^{N}, R^{\prime}\right)=\bar{M}\left(R^{N}, R^{\prime}\right)=\{a, c\}$ and $\bar{F}\left(R^{N}, R^{\prime}\right)=c$, hence a violation of TP.

Proof of the claims in Example 4.5 We first prove TP of $\breve{F}$. Let $R^{N}$ be a profile with $x=\breve{F}\left(R^{N}\right)$ and let $R^{\prime} \in L$ with $t\left(R^{\prime}\right)=x$. We have to show that $\breve{F}\left(R^{N}, R^{\prime}\right)=x$. Note that $x \in \check{M}\left(R^{N}, R^{\prime}\right)$.

If $y \in A \backslash M\left(R^{N}\right)$ then $m_{y}\left(R^{N}\right)>m_{x}\left(R^{N}\right)$, hence $m_{y}\left(R^{N}, R^{\prime}\right) \geq m_{y}\left(R^{N}\right)-1>$ $m_{x}\left(R^{N}\right)-1=m_{x}\left(R^{N}, R^{\prime}\right)$, so that $y \notin M\left(R^{N}, R^{\prime}\right)$. Hence, $M\left(R^{N}, R^{\prime}\right) \subseteq M\left(R^{N}\right)$. 
We claim that $\check{M}\left(R^{N}, R^{\prime}\right) \subseteq \check{M}\left(R^{N}\right)$. To prove this, let $z \in A \backslash \check{M}\left(R^{N}\right)$. We show that $z \notin \check{M}\left(R^{N}, R^{\prime}\right)$. If $z \notin M\left(R^{N}, R^{\prime}\right)$, then $z \notin \check{M}\left(R^{N}, R^{\prime}\right)$ and we are done. Now suppose $z \in M\left(R^{N}, R^{\prime}\right)$, hence also $z \in M\left(R^{N}\right)$ by the preceding paragraph. If $b\left(R^{\prime}\right)=z$ then $m_{z}\left(R^{N}, R^{\prime}\right)=m_{z}\left(R^{N}\right)+1=m_{x}\left(R^{N}\right)+1>m_{x}\left(R^{N}\right)-1=m_{x}\left(R^{N}, R^{\prime}\right)$, so that $z \notin M\left(R^{N}, R^{\prime}\right)$, a contradiction. Hence $b\left(R^{\prime}\right) \neq z$ and therefore $\beta_{z}\left(R^{N}, R^{\prime}\right)=$ $\beta_{z}\left(R^{N}\right)<\beta_{x}\left(R^{N}\right)=\beta_{x}\left(R^{N}, R^{\prime}\right)$, where the strict inequality follows from $z \in M\left(R^{N}\right) \backslash$ $\check{M}\left(R^{N}\right)$ (with $\beta$-notation as in the preceding proof). Hence we have $z \in M\left(R^{N}, R^{\prime}\right) \backslash$ $\check{M}\left(R^{N}, R^{\prime}\right)$. This concludes the proof of the claim.

Since $x$ is the maximal element of $\check{M}\left(R^{N}\right)$ according to ranking $Q$, the above claim implies that it is also the maximal element of $\check{M}\left(R^{N}, R^{\prime}\right)$ according to ranking $Q$, and therefore $\breve{F}\left(R^{N}, R^{\prime}\right)=x$, as was to be proved.

To show that $\breve{F}$ does not satisfy BP we exhibit the following example of a 12-voter profile $R^{N}$ with set of alternatives $A=\{a, b, c, d, e\}$, and $Q=a b c d e$ :

\begin{tabular}{llllll}
4 & 1 & 1 & 1 & 1 & 4 \\
\hline$b$ & $b$ & $c$ & $c$ & $a$ & $a$ \\
$e$ & $c$ & $d$ & $d$ & $c$ & $d$ \\
$c$ & $d$ & $e$ & $e$ & $d$ & $e$ \\
$d$ & $e$ & $b$ & $a$ & $e$ & $c$ \\
$a$ & $a$ & $a$ & $b$ & $b$ & $b$
\end{tabular}

Here, $M\left(R^{N}\right)=\check{M}\left(R^{N}\right)=\{a, b\}$ and $\check{F}\left(R^{N}\right)=a$. Add a ranking $R^{\prime}=c d e a b$ to obtain the profile $\left(R^{N}, R^{\prime}\right)$ given by

\begin{tabular}{llllll}
4 & 1 & 1 & 2 & 1 & 4 \\
\hline$b$ & $b$ & $c$ & $c$ & $a$ & $a$ \\
$e$ & $c$ & $d$ & $d$ & $c$ & $d$ \\
$c$ & $d$ & $e$ & $e$ & $d$ & $e$ \\
$d$ & $e$ & $b$ & $a$ & $e$ & $c$ \\
$a$ & $a$ & $a$ & $b$ & $b$ & $b$
\end{tabular}

then $M\left(R^{N}, R^{\prime}\right)=\{a, b, c\}$ and $\check{M}\left(R^{N}, R^{\prime}\right)=\{b\}$, hence $\check{F}\left(R^{N}, R^{\prime}\right)=b$, which is a violation of BP.

Proof of the claims in Example 4.6 We first show that $F$ satisfies BP. Due to Proposition 4.1 we only have to consider cases in which (i) $b$ is not chosen but by adding a ranking with $b$ at bottom we obtain profile $R^{*}$ and (ii) the cases in which we add to $R^{*}$ a new ranking with bottom alternative $a$ or $c$. 
For (i) let $N=\{1, \ldots, 9\}$ and first consider profile $R_{1}^{N}$ given by

\begin{tabular}{ccc}
3 & 3 & 3 \\
\hline$a$ & $b$ & $c$ \\
$b$ & $c$ & $a$ \\
$c$ & $a$ & $b$
\end{tabular}

Then $M\left(R_{1}^{N}\right)=\{a, b, c\}$ and $F\left(R_{1}^{N}\right)=b$. Hence, this case cannot lead to a violation of BP.

Next consider the profile $R_{2}^{N}$ as follows:

\begin{tabular}{lll}
2 & 3 & 4 \\
\hline$a$ & $b$ & $c$ \\
$b$ & $c$ & $a$ \\
$c$ & $a$ & $b$
\end{tabular}

Then $M\left(R_{2}^{N}\right)=\{c\}$ and $F\left(R_{2}^{N}\right)=c$. Now $R^{*}$ is obtained by adding the ranking $R^{\prime}=a b c$ but since $b\left(R^{\prime}\right)=c$ this does not lead to a violation of BP.

Now consider the following profile $R_{3}^{N}$ :

\begin{tabular}{lll}
3 & 2 & 4 \\
\hline$a$ & $b$ & $c$ \\
$b$ & $c$ & $a$ \\
$c$ & $a$ & $b$
\end{tabular}

Then $M\left(R_{3}^{N}\right)=\{c\}$ and $F\left(R_{3}^{N}\right)=c$. Since $R^{*}$ is obtained by adding the ranking $R^{\prime}=b c a$, again this does not lead to a violation of BP.

For (ii), let $N=\{1, \ldots, 10\}$ and consider $R^{*}$, thus $F\left(R^{*}\right)=b$. Add a ranking $R^{\prime}$ with $b\left(R^{\prime}\right)=c$. If $b R^{\prime} a R^{\prime} c$ then $M\left(R^{*}, R^{\prime}\right)=\{a, b, c\}$ and $F\left(R^{*}, R^{\prime}\right)=b \neq c$. If $a R^{\prime} b R^{\prime} c$ then $M\left(R^{*}, R^{\prime}\right)=\{a, c\}$ and $F\left(R^{*}, R^{\prime}\right)=a \neq c$. Since $a \notin\{b, c\}=H\left(R^{*}\right)$ and $H$ satisfies BP, we do not have to consider rankings $R^{\prime}$ such that $b\left(R^{\prime}\right)=a$. We conclude that $F$ satisfies BP.

Now we show TP of $F$. As in the case of BP, first consider 9-voter profiles which may result in $R^{*}$ by adding a ranking. In the case of $R_{1}^{N}$ we have $F\left(R_{1}^{N}\right)=b$ and $R^{*}$ is obtained by adding the ranking $R^{\prime}=c a b$, which does not violate TP. For $R_{2}^{N}$ we have $F\left(R_{2}^{N}\right)=c$ and $R^{*}$ is obtained by adding the ranking $R^{\prime}=a b c$, which does not violate TP. For $R_{2}^{N}$ we have $F\left(R_{3}^{N}\right)=c$ and $R^{*}$ is obtained by adding the ranking $R^{\prime}=b c a$, again no violation of TP.

Finally, consider $R^{*}$ with $F\left(R^{*}\right)=b$. Adding the ranking $R^{\prime}=b a c$ results in $M\left(R^{*}, R^{\prime}\right)=\{a, b, c\}$ and $F\left(R^{*}, R^{\prime}\right)=b$, and adding the ranking $R^{\prime}=b c a$ results 
in $M\left(R^{*}, R^{\prime}\right)=\{b, c\}$ and $F\left(R^{*}, R^{\prime}\right)=b$, hence no violation of TP. All other cases follow from Proposition 4.1 .

Proof of the claims in Example 4.7 Let $R^{\prime}=a b c$. Then $M\left(R^{*}, R^{\prime}\right)=\{a, c\}$, hence $F\left(R^{*}, R^{\prime}\right)=c$, which violates BP since $F\left(R^{*}\right)=b$.

The proof of TP of $F$ is similar to the proof above for Example 4.6. We have $F\left(R_{1}^{N}\right)=b$, so this cannot violate TP. For $R_{2}^{N}$ we have $F\left(R_{2}^{N}\right)=c$ and therefore adding $R^{\prime}=a b c$ to obtain $R^{*}$ cannot violate TP. For $R_{3}^{N}$ we have $F\left(R_{3}^{N}\right)=c$ and therefore adding $R^{\prime}=b c a$ to obtain $R^{*}$ cannot violate TP either. Considering $R^{*}$ and adding $R^{\prime}=b a c$ results in $M\left(R^{*}, R^{\prime}\right)=\{a, b, c\}$ and $F\left(R^{*}, R^{\prime}\right)=b$, which does not violate TP. Considering $R^{*}$ and adding $R^{\prime}=b c a$ results in $M\left(R^{*}, R^{\prime}\right)=\{b, c\}$ and $F\left(R^{*}, R^{\prime}\right)=b$, which again does not violate TP. All other cases follow from Proposition 4.1 .

Proof of the claims in Example 4.8 A violation of TP is obtained by adapting the profile used for this purpose in the proof of the claims in Example 4.4. (Another violation of TP can be obtained by adding the ranking $R^{\prime}=b a c$ to $R^{*}$, since then $F\left(R^{*}, R^{\prime}\right)=a \neq b=F\left(R^{*}\right)$, see below.) In order to show BP it is by the proof of the claims in Example 4.4 again sufficient to consider the cases involving $R^{*}$. First, $F\left(R_{1}^{N}\right)=b$, which cannot lead to a violation of BP. Second, $F\left(R_{2}^{N}\right)=c$, hence adding $R^{\prime}=a b c$ to obtain $R^{*}$ does not lead to a violation of BP. Third, $F\left(R_{3}^{N}\right)=c$, hence adding $R^{\prime}=b c a$ to obtain $R^{*}$ does not lead to a violation of BP. Finally, consider $R^{*}$ with $F\left(R^{*}\right)=b$. Adding a ranking $R^{\prime}$ with $b\left(R^{\prime}\right)=a$ will result in $F\left(R^{*}, R^{\prime}\right)=c$; and adding a ranking $R^{\prime}$ with $b\left(R^{\prime}\right)=c$ will result in $F\left(R^{*}, R^{\prime}\right)=a$. Hence, no violations of BP occur.

Proof of Proposition 4.9 First, we show that $\widetilde{H}$ satisfies WCC. Let $R^{N} \in L^{N}$ such that $\mathcal{C}_{s}\left(R^{N}\right) \neq \emptyset$. Then $\mathcal{C}_{s}\left(R^{N}\right)=M\left(R^{N}\right) \subseteq \widetilde{H}\left(R^{N}\right)$. Suppose that $x \in A \backslash \mathcal{C}_{s}\left(R^{N}\right)$. Take $y \in \mathcal{C}_{s}\left(R^{N}\right)$. Then $m_{y}\left(R^{N}\right)<0<m_{x}\left(R^{N}\right)$. Hence $n_{x y}\left(R^{N}\right)<0$, which is equivalent to $n_{y x}\left(R^{N}\right)>0$. Thus, $n_{y x}\left(R^{N}\right)>m_{y}\left(R^{N}\right)$, so that $x \notin \widetilde{H}\left(R^{N}\right)$.

Next we show that $\widetilde{H}$ satisfies TP. Let $R^{N}$ be a profile and $x \in A$ with $x \in \widetilde{H}\left(R^{N}\right)$. Let $R^{\prime} \in L$ with $t\left(R^{\prime}\right)=x$. First, if $\mathcal{C}_{s}\left(R^{N}\right) \neq \emptyset$, then $\widetilde{H}\left(R^{N}\right)=\mathcal{C}_{s}\left(R^{N}\right)=M\left(R^{N}\right)$ by the first part of the proof. Then, by Proposition 4.1, $x \in M\left(R^{N}, R^{\prime}\right)$, hence $x \in$ $\widetilde{H}\left(R^{N}, R^{\prime}\right)$ and TP is satisfied. Second, if $\mathcal{C}_{s}\left(R^{N}\right)=\emptyset$, then suppose there is $y \in A$ with $m_{y}\left(R^{N}, R^{\prime}\right)<m_{x}\left(R^{N}, R^{\prime}\right)$. We have to show that $n_{y x}\left(R^{N}, R^{\prime}\right) \leq m_{y}\left(R^{N}, R^{\prime}\right)$. If $m_{y}\left(R^{N}\right)<m_{x}\left(R^{N}\right)$ then $n_{y x}\left(R^{N}, R^{\prime}\right)=n_{y x}\left(R^{N}\right)-1 \leq m_{y}\left(R^{N}\right)-1 \leq m_{y}\left(R^{N}, R^{\prime}\right)$, where the first weak inequality follows since $x \in \widetilde{H}\left(R^{N}\right)$. If $m_{y}\left(R^{N}\right) \geq m_{x}\left(R^{N}\right)$ then $m_{x}\left(R^{N}, R^{\prime}\right)>m_{y}\left(R^{N}, R^{\prime}\right) \geq m_{y}\left(R^{N}\right)-1 \geq m_{x}\left(R^{N}\right)-1=m_{x}\left(R^{N}, R^{\prime}\right)$, a contradiction, so this case cannot occur. Lastly, we show that $\widetilde{H}$ satisfies BP. Let $R^{N} \in L^{N}$, $R^{\prime} \in L$ with $b\left(R^{\prime}\right)=x$, and suppose that $x \in \widetilde{H}\left(R^{N}, R^{\prime}\right)$. We show that $x \in \widetilde{H}\left(R^{N}\right)$. Suppose $m_{y}\left(R^{N}\right)<m_{x}\left(R^{N}\right)$ for some $y \in A$. Then $m_{y}\left(R^{N}, R^{\prime}\right)<m_{x}\left(R^{N}, R^{\prime}\right)$. 
Since $x \in \widetilde{H}\left(R^{N}, R^{\prime}\right)$ this implies $n_{y x}\left(R^{N}, R^{\prime}\right) \leq m_{y}\left(R^{N}, R^{\prime}\right)$, hence $n_{y x}\left(R^{N}\right)+1 \leq$ $m_{y}\left(R^{N}, R^{\prime}\right)$. This implies $n_{y x}\left(R^{N}\right) \leq m_{y}\left(R^{N}, R^{\prime}\right)-1 \leq m_{y}\left(R^{N}\right)+1-1=m_{y}\left(R^{N}\right)$. Hence, $x \in \widetilde{H}\left(R^{N}\right)$.

Proof of Proposition 4.10 Let $R^{N} \in L^{N}$ and suppose that for some $x \in A$ we have $x \in C\left(R^{N}\right) \backslash \widetilde{H}\left(R^{N}\right)$. We derive a contradiction. Since $x \notin \widetilde{H}\left(R^{N}\right)$, there is a $y \in A$ such that $m_{y}\left(R^{N}\right)<m_{x}\left(R^{N}\right)$ and $n_{y x}\left(R^{N}\right)>m_{y}\left(R^{N}\right)$. By WCC of $C$ we must have $m_{y}\left(R^{N}\right) \geq 0$. Hence, $n_{y x}\left(R^{N}\right) \geq 0$. Equivalently, $n_{x y}\left(R^{N}\right)<0$, which implies that $m_{y}\left(R^{N}\right)=n_{\widetilde{x} y}\left(R^{N}\right)$ for some $\widetilde{x} \in A \backslash\{x\}$, and in particular, $x$ does not have the maximal resistance against $y$ at the profile $R^{N}$. Let $R^{\prime} \in L$ such that $x R^{\prime} y R^{\prime} z$ for all $z \in A \backslash\{x, y\}$. Then $m_{x}\left(R^{N}, R^{\prime}\right)=m_{x}\left(R^{N}\right)-1$ and $m_{y}\left(R^{N}, R^{\prime}\right)=m_{y}\left(R^{N}\right)-$ 1. Thus, we still have $m_{y}\left(R^{N}, R^{\prime}\right)<m_{x}\left(R^{N}, R^{\prime}\right)$, and $n_{y x}\left(R^{N}, R^{\prime}\right)=n_{y x}\left(R^{N}\right)-$ $1>m_{y}\left(R^{N}\right)-1=m_{y}\left(R^{N}, R^{\prime}\right)$. Therefore, if we keep on adding the ranking $R^{\prime}$, then $n_{y x}\left(R^{N}, R^{\prime}, \ldots, R^{\prime}\right)$ decreases but still $n_{y x}\left(R^{N}, R^{\prime}, \ldots, R^{\prime}\right)>m_{y}\left(R^{N}, R^{\prime}, \ldots, R^{\prime}\right)$. Hence, after adding $m_{y}\left(R^{N}\right)+1$ rankings $R^{\prime}$ we have $m_{y}\left(R^{N}, R^{\prime}, \ldots, R^{\prime}\right)<0$ while $m_{x}\left(R^{N}, R^{\prime}, \ldots, R^{\prime}\right)>m_{y}\left(R^{N}, R^{\prime}, \ldots, R^{\prime}\right)$. Then by WCC, $y \in C\left(R^{N}, R^{\prime}, \ldots, R^{\prime}\right)$ and $x \notin C\left(R^{N}, R^{\prime}, \ldots, R^{\prime}\right)$. This violates TP of $C$. 


\subsection{INTRODUCTION}

An abstract game consists of a set of states (or outcomes), agents' utilities in each state, and an effectivity correspondence that specifies for any two states what coalitions are effective for a move from one to the other, i.e. what coalitions can enforce such a move. In particular, an effectivity correspondence does not specify strategies for any player; specifically, it abstracts away any strategic interaction.

Solutions of abstract games heavily rely on dominance: one state dominates another state if there is a coalition that (i) is effective for moving from the latter to the former, and (ii) thereby achieves a strictly better outcome for all their members. Arguably, the most prominent solutions based on dominance are stable sets (von Neumann \& Morgenstern, 1944) and the core (Gillies, 1959). Both solutions presuppose myopic behavior: (a) whenever a coalition deviates to a new state, they expect to remain in that state; (b) whenever a coalition does not deviate to a new state, they expect to remain in the old state. That is, coalitions expect that no other coalition will ever implement a change. While assumption (a) has already been weakened in the literature on farsighted behavior, assumption (b) has been criticized (for instance by Chwe, 1994), but a convincing solution has not been offered yet. This chapter attempts to do so, that is, we propose a way to close this gap in the theory of farsightedness.

Myopic behavior is a severe restriction of agents' rationality, and the whole dilemma comes to light if one inspects stable sets of von Neumann \& Morgenstern (1944). A stable set is a set of outcomes that satisfies internal stability-no outcome inside the stable set dominates any other outcome inside — and external stability — every outcome outside the stable set is dominated by some outcome inside. It is, however, possible that a state outside dominates a state inside. According to von Neumann \& Morgenstern (1944) no coalition would implement this state outside because another coalition would implement a move back into the stable set right away. This argument is surely valid for single-payoff stable sets, i.e. stable sets in which all states deliver the same payoff vector. However, for situations where payoff vectors are different Harsanyi (1974) provided the following thought: a coalition $S$ could deviate from a state $x$ inside the stable set to a state $y$ outside, in anticipation of another coalition's deviating from $y$ to third state $z$ 
inside. If $z$ is preferred over $x$ by all members of $S$ then $S$ would actually be quite happy with this development, so there is an indirect dominance of $z$ over $x$.

Chwe (1994) formalized this indirect dominance relation, according to which coalitions only care about the final outcome of a chain of deviations. This takes, at least in parts, care of criticism (a) above: coalitions do not expect to remain in the state they deviate to. Chwe's formulation was quite successful and in the sequel a whole branch of literature emerged: Xue (1998) endowed agents with expectations about each others' behavior based on whether they are optimistic or pessimistic, Diamantoudi \& Xue (2003) consider farsightedness in hedonic games, Herings et al. (2009) apply farsightedness to problems of network formation, and Mauleon et al. (2011) are interested in stable sets in matching problems when players are farsighted.

Each of the proposed solutions (which we will describe in more detail and compare to our approach in Section 5.4) relies on an indirect dominance relation according to which a state is dominated if there is some indirect dominance path to another state. However, none of them requires that this final state is, in fact, not indirectly dominated-which is why they only partially solve criticism (a). More recent approaches have explicitly tackled this problem. Jordan (2006) introduced expectation functions for pillage games. Adapted to abstract games, these functions specify for each state what (unique) coalition moves to what (unique) state. If such a function is commonly known, then coalitions know exactly what will happen after any potential move, and a dominance relation can be based on this expectation. Of course there should be some coherence between the expectation function itself and the dominance relation it creates, in the sense that moves should only be expected if they are beneficial. Interpreting the stationary points of such a function as stable outcomes seems natural and finally solves problem (a) above. This approach has been taken by Dutta \& Vohra (2017) who introduce three attractive axioms an expectation function might satisfy; the case of heterogeneous expectations has recently been explored by Bloch \& van den Nouweland (2017).

Problem (b), however, persists: in the model of Dutta \& Vohra (2017) agents compare the final outcome of a deviation to the status quo, making the implicit assumption that the status quo prevails if they do not deviate. We illustrate the resulting issues in Section 5.3, where we discuss their model in more detail. The crucial point is that agents are farsighted enough to consider what happens if they deviate, but not farsighted enough to consider what happens if they do not move (cf. Chwe, 1994).

The first goal of this chapter, which we pursue in Section 5.3, is to adapt the model of Dutta \& Vohra (2017) in order to solve this issue: an extended expectation function specifies for each state an ordered list of coalitions and their moves, and each coalition knows that if they don't move, the next one will. This knowledge allows coalitions to compare the result of their move to the scenario of not moving. Of course, there might be different orders in which coalitions are allowed to move, and different orders might 
lead to different behavior and different stationary points. Yet, this non-uniqueness also emerges in the model of Dutta \& Vohra (2017) from specifying what coalition is allowed to move at a given state (if there are more than one coalition that could move according to the rules of the game). Hence, we might end up with different solutions, but each solution is supported by at least one specification of how the game is being played.

We impose three axioms on these extended expectation functions and call a function rational if it satisfies all of them:

INTERNAL STABILITY. The only reason for a coalition $T$ not to implement a move out of a state is that there is a different coalition $S$ whose move out of this state is at least as beneficial for at least one member of $T$ as $T$ 's move. This member would simply not support any such move and rather wait for $S$ to move.

EXTERNAL STABILITY. If a coalition does move, it must be strictly better for all members than not moving and leaving the floor to the next coalition. Here, we explicitly address problem (b) by allowing a comparison between deviating and not deviating without assuming that the latter would secure the status quo.

MAXIMALITY. If a coalition moves to some state, there is no other move for which they are effective and which is strictly better for all members.

Note that these axioms are independent of the order in which coalitions move; nevertheless, they might be satisfied for some order, but not for others. We follow the approach of Dutta \& Vohra (2017) and interpret the stationary points of these rational extended expectation functions as stable outcomes.

Most criticism about dominance relations, including ours, is based on implied bounds on the rationality of coalitions or players. But this problem naturally arises from different languages: dominance is defined in a very abstract setting without strategic interaction, whereas the criticism is formulated in terms of behavior and, hence, strategies. In order to reconcile these two worlds it seems natural to motivate a dominance relation by supporting it in a non-cooperative fashion. This shall be the second aim of this chapter, which we pursue in Section 5.5. An abstract game is, essentially, a coalition formation game, and the existing literature on non-cooperative coalition formation is quite rich (cf. Ray, 2007). But: the translation of an abstract game into a non-cooperative coalition formation game requires the specification of an extensive form, and, as Ray \& Vohra (2017) point out, a solution might then very much depend on the choice of this extensive form.

We shall circumvent this issue: instead of providing an extensive form in which players might have very complicated strategies, we endow coalitions with rather simple strategies. A coalition's strategy specifies for any state of the abstract game whether they 
will remain there or deviate to another state for which they are effective. This setup is similar to Kimya (2018) who provides a non-cooperative foundation for the model of Dutta \& Vohra (2017).

The translation between coalitions' strategies and extended expectation functions is straightforward. Suppose an order $\succeq$ in which coalitions are allowed to move in each state is given. Then for each state, one constructs a list of coalitions by selecting all those coalitions whose strategies specify a move out of this state, and one orders this list according to $\succeq$. So, strategy profiles build a natural foundation for extended expectation functions.

We define a best response of a coalition $S$ as a vector of strategies, one strategy for each nonempty subcoalition of $S$, such that (i) each proper subcoalition plays a best response, (ii) the strategy of $S$ is supported by all players, and (iii) $S$ cannot implement any move that would be strictly better for all members. An equilibrium is then a strategy profile in which each coalition plays a best response, or equivalently, which is a best response for the grand coalition. Our main result is that an extended expectation function satisfies our axioms if and only if it corresponds to an equilibrium in the associated non-cooperative coalition formation game. Hence, we call the set of stationary points of a rational extended expectation function equilibrium stable set.

In Section 5.6 we apply our solution to various games such as games in characteristic function form and two-sided one-to-one matching problems. We provide a brief conclusion and avenues for further research in Section 5.7.

\subsection{PRELIMINARIES}

\subsubsection{Notation}

Let $N$ be a finite set of players. Subsets $S \subseteq N$ are called coalitions. For $S \subseteq N$ write $2^{S}$ for the set of subsets of $S$, and $P(S)$ for the set of nonempty subsets. An abstract game is a tupel $\left(N, X, E,\left(U_{i}(\cdot)\right)_{i \in N}\right)$, where $X$ is the set of outcomes or states, $U_{i}: X \rightarrow \mathbb{R}$ is player $i$ 's utility function over states, and $E: X \times X \rightrightarrows 2^{N}$ is an effectivity correspondence: for two states $x, y \in X$ the (possible empty) set $E(x, y)$ comprises all coalitions that are effective for a move from $x$ to $y$, i.e. that can replace $x$ with $y$. We assume that $E(x, x)=2^{N}$, that is, each coalition has the option not to change the status quo; and $\emptyset \in E(x, y)$ if and only if $x=y$. 


\subsubsection{Expectation functions}

Let $\left(N, X, E,\left(U_{i}(.)\right)_{i \in N}\right)$ be an abstract game. A (basic) expectation function is a map $F: X \rightarrow X \times 2^{N}$ with $F(x)=(f(x), S(x))$ such that $S(x) \in E(x, f(x)) . F$ describes transitions from every state to either itself or another state: $f(x)$ is the state that is transitioned to, and $S(x)$ is the coalition that implements the transition (in particular, $S(x)$ is effective for a move from $x$ to $f(x)$ ). We follow the standard convention and require $S(x)=\emptyset$ whenever $f(x)=x$. Given an expectation function $F$ and a state $x \in X$, a path $P=P_{F}(x)$ is a finite or infinite sequence of states $\left(x^{1}, x^{2}, \ldots\right)$ with $x^{1}=x, x^{k+1}=f\left(x^{k}\right)$ for all $k \in \mathbb{N}$. $P$ is terminal if there is $m<\infty$ such that $f\left(x^{m}\right)=x^{m} \cdot{ }^{1}$ In this case we write $t\left(P_{F}(x)\right)$ to denote the terminal node of $P_{F}$, i.e. $t\left(P_{F}(x)\right)=x^{m}$. An expectation function $F$ is absorbing if $P_{F}(x)$ is terminal for all $x \in X$.

\subsection{RATIONAL EXPECTATIONS}

\subsubsection{Rational expectation functions}

Let $F$ be an expectation function. We assume that players only care about their utility in terminal nodes of $P_{F}$, and that any terminal node is better than reaching no terminal node at all (cf. Harsanyi, 1974). So, for $i \in N$ let

$$
u_{i}(x, F)= \begin{cases}U_{i}\left(t\left(P_{F}(x)\right)\right) & \text { if } P_{F}(x) \text { is terminal } \\ -\infty & \text { otherwise }\end{cases}
$$

If $F$ is absorbing then $u_{i}(x, F)=U_{i}\left(t\left(P_{F}(x)\right)\right)$ for all $x \in X$. The following properties an absorbing expectation function may satisfy have been introduced by Dutta \& Vohra (2017).

INTERNAL STABILITY, I'. For all $x \in X$, if $f(x)=x$ then for all $\emptyset \neq T \subseteq N$ and all $y \in X$ with $T \in E(x, y)$ there is $i \in T$ such that $U_{i}(x) \geq u_{i}(y, F)$.

EXternal StABility, E'. For all $x \in X$, if $f(x) \neq x$ then $u_{i}(x, F)>U_{i}(x)$ for all $i \in S(x)$.

MAXIMALITY, M'. For all $x \in X$, if $f(x) \neq x$ and there is $y \neq x$ with $S(x) \in E(x, y)$ then there is $i \in S(x)$ such that $u_{i}(x, F) \geq u_{i}(y, F)$.

\footnotetext{
${ }^{1}$ If $P$ is not terminal, $P$ is either an infinite path or contains a cycle.
} 
StRONG MAXIMALity, SM'. For all $x \in X$, if $f(x) \neq x$ and there are $y \neq f(x)$ and $T \in E(x, y)$ with $T \cap S(x) \neq \emptyset$ then there is $i \in T$ such that $u_{i}(x, F) \geq u_{i}(y, F)$.

I' ensures that if no coalition moves out of a state $x$, then there is no coalition that could profit by doing so. E' ensures that a coalition only moves out of a state $x$ if the terminal node is strictly preferred over $x$ by all members. ${ }^{2}$ M' requires that deviations are optimal; that is, the deviating coalition $S(x)$ is not effective for a move to any state that is strictly better for all members. Finally, SM' requires, additionally, that no subgroup of the deviating coalition $S(x)$ could improve by joining some other coalition instead of $S(x)$ : such a subgroup would have no reason to support the move of $S(x)$.

An absorbing expectation function is called rational if it satisfies I', E', and M'; it is called strongly rational if it satisfies I', E', and SM'. The set of terminal nodes of a rational expectation function is called a rational expectations farsighted stable set (REFS), and the set of terminal nodes of a strongly rational expectation function is called a strongly rational expectations farsighted stable set (SREFS).

While I' and $\mathbf{M}$ ' are rather uncontroversial, $\mathbf{E}$ ' is not as innocuous as it seems: condition $u_{i}(x, F)>U_{i}(x)$ for all $i \in S(x)$ makes the implicit assumption that players in $S(x)$ expect the status quo to prevail in case they don't move. But, putting it in the words of Chwe (1994):

This is clearly inconsistent. A coalition is farsighted enough to consider what further moves other coalitions will make once it moves, but does not consider what other coalitions will do if it does not move.

Example 5.1. Let $N=\{1,2\}, X=\{x, y, z\}$ and consider the effectivity correspondence and payoffs depicted in Figure 2(a). Suppose that $F(x)=(y,\{1\})$. This clearly violates $\boldsymbol{E}^{\prime}$, as $\{1\}$ reduces her payoff from 2 to 1 . Nevertheless, if she stayed in $x$, it is very likely that $\{2\}$ takes the opportunity and moves to $z$, reducing 1 's payoff even further. Hence, $\{1\}$ 's move from $x$ to $y$ is the choice of the lesser evil.

In the previous example agent 1's optimal move crucially depends on her expectation about agent 2's behavior if she did not move. But these "counterfactuals" cannot be described by an expectation function.

Suppose for a moment, we accept the reasoning that motivates E'. Another issue arises upon closer inspection of SM' if we do so. According to SM' a move by a coalition $S$ is forbidden if there is some coalition $T$ that intersects with $S$ and that could move to a different state $y$ such that the longterm outcome following $y$, i.e. $t\left(P_{F}(y)\right)$, is preferred over the longterm outcome following $f(x)$, i.e. $t\left(P_{F}(x)\right)$, by all members of $T$. (Recall

\footnotetext{
${ }^{2}$ Dutta \& Vohra (2017) require that $P_{F}(x)$ is a farsighted objection against $x$. But since External Stability is required in all non-terminal nodes of the path $P_{F}(x)$, this is equivalent.
} 
Figure 1: External stability and maximality revisited

(a) Should 1 move to $y$ ?

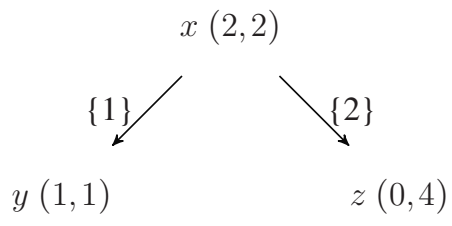

(b) Should 2 boycott a move to $x$ ?

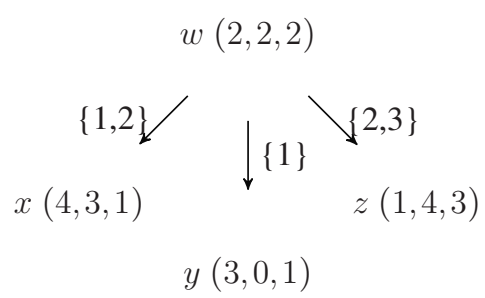

$y(3,0,1)$

that $t\left(P_{F}(f(x))\right)=t\left(P_{F}(x)\right)$ by definition.) In this instance the members of $T \backslash S$ compare their payoff from joining $S \cap T$ and implementing a move to $y$ to not joining $S \cap T$ and letting $S$ implement a move to $f(x)$. So, they compare the longterm outcome of moving to the longterm outcome of not moving. This is hard to reconcile with the presumption that underlies E'.

A last issue that arises from SM' is the following: it is assumed that the members of $S \cap T$ will boycott the move of $S$ and instead try to implement, together with $T \backslash S$, the move to $y$. But is it clear that such a boycott brings the expected result? ${ }^{3}$

Example 5.2. Let $N=\{1,2,3\}, X=\{w, x, y, z\}$ and consider the effectivity correspondence and payoffs depicted in Figure 2(b). SM' requires $F(w) \neq(x,\{1,2\})$ on the grounds that 2 could, together with 3 , beneficially implement a move to $z$. However, if 2 boycotts $x$, she can by no means be sure that $z$ will actually be implemented as $\{1\}$ might move to y instead.

The optimal behavior in the previous example depends, again, on counterfactuals: what happens if $\{1,2\}$ does not implement $x$ ? In the setup of Dutta \& Vohra (2017) agent 2 pays no attention to $\{1\}$ 's options and assumes instead that she will be able to implement $z$ together with 3. A basic expectation function cannot even represent a more sophisticated reasoning of agent 2. Yet, in order to analyze farsighted behavior properly, it is essential to depict expectations about what happened if a coalition did not change the status quo. We therefore extend expectation functions so as to incorporate these counterfactuals in the next subsection.

\footnotetext{
${ }^{3}$ The issue of maximality has recently been tackled by Ray \& Vohra (2017).
} 


\subsubsection{Extended expectation functions}

Definition 5.1. An extended expectation function is a map $F$ that assigns to each $x \in X$ an ordered list $\left(F^{1}(x), \ldots, F^{k(x)}(x)\right)$ such that $F^{l}(x)=\left(f^{l}(x), S^{l}(x)\right) \in X \times 2^{N}$ with $S^{l}(x) \in E\left(x, f^{l}(x)\right)$ for all $l=1, \ldots, k(x), S^{l}(x) \neq S^{l^{\prime}}(x)$ for all $l \neq l^{\prime}, f^{l}(x) \neq$ $x$ for all $l \neq k(x)$, and $S^{k(x)}(x)=\emptyset$.

Observe that this definition implies $S^{l}(x) \neq \emptyset$ for $l \neq k(x)$ and $f^{k(x)}(x)=x$. Both the length and the order of the list $F(x)$ might depend on $x$. These ordered lists allow us to incorporate the counterfactuals outlined above: if $S^{1}(x)$ did not move to $f^{1}(x)$ then $S^{2}(x)$ would move to $f^{2}(x)$. Note, however, that each coalition is allowed to move at most once.

For any extended expectation function $F$ the map $F^{1}$ is a basic expectation function, which reflects the "true behavior" in each node. In particular, $F$ induces the path $P_{F^{1}}(x)$ for all $x \in X$. As there is no danger of confusion, we shall simply write $P_{F}(x)$ to refer to this path, even if $F$ is an extended expectation function. In particular, for an extended expectation function $F$ we shall write $u_{i}(x, F)$ for $u_{i}\left(x, F^{1}\right)$.

In the following we adapt the axioms of Dutta \& Vohra (2017) so that they apply to extended expectation functions. It is worth mentioning, though, that with the definition of the utility function in Equation (9), these axioms are well defined for $F$ even if $F^{1}$ is not absorbing.

InTERnAL STAB ILITY, I. For all $x \in X$ and all coalitions $T \notin\left\{S^{1}(x), \ldots, S^{k(x)}(x)\right\}$ there is $l \leq k(x)$ such that for each $y \in X$ with $T \in E(x, y)$ there is $i \in T$ with $u_{i}\left(f^{l}(x), F\right) \geq u_{i}(y, F)$.

EXTERnAL STABILITY, E. For all $x \in X$ and for all $l=1, \ldots, k(x)-1$ it holds that $u_{i}\left(f^{l}(x), F\right)>u_{i}\left(f^{l+1}(x), F\right)$ for all $i \in S^{l}(x)$.

MAXIMALITY, M. For all $x \in X$ and for all $l=1, \ldots, k(x)-1$ it holds that if there is $y \neq f^{l}(x)$ such that $S^{l}(x) \in E(x, y)$ then there is $i \in S^{l}(x)$ with $u_{i}\left(f^{l}(x), F\right) \geq$ $u_{i}(y, F)$.

Internal stability is a requirement on all coalitions $T$ that do not want to move out of a state $x$ : there must be a move by some coalition $S^{l}(x)$, such that whatever change $T$ could implement, at least one member would not agree to that change. External stability takes into consideration our previous discussion. A coalition $S^{l}(x)$ that moves out of $x$ does not compare the final outcome this move entails to the payoff vector obtained in $x$, but to the payoff vector that would be reached if the next coalition, $S^{l+1}(x)$, were allowed to move. Maximality requires that any moving coalition moves to an optimal 
Figure 3: External stability

$$
\begin{gathered}
y(4,1,0,3) \\
\{2\} \uparrow\{4\} \\
w(3,0,0,0) \stackrel{\{1\}}{\longrightarrow} x(2,2,2,2) \stackrel{\{3\}}{\longrightarrow} z(2,3,1,0)
\end{gathered}
$$

state, in the sense that there is no other state they could move to which would make all members strictly better off.

An extended expectation function satisfying $\mathbf{I}, \mathbf{E}$, and $\mathbf{M}$ is called rational. A stationary point of an extended expectation function $F$ is a state $x$ such that $f^{1}(x)=x$. The set of stationary points of a rational extended expectation function $F$, denoted by $\mathcal{S}(F)$, is said to be an equilibrium stable set (ESS). A justification for the name will be given in Section 5.5, where we shall provide a non-cooperative foundation for rational extended expectation functions.

Example 5.3. Consider the game depicted in Figure 3. Consider $F$ with $F^{1}(w)=$ $(x,\{1\}), F^{1}(x)=(y,\{2\}), F^{2}(x)=(z,\{3\})$, and $F^{3}(x)=(y,\{4\})$. This extended expectation function ${ }^{4}$ satisfies $\boldsymbol{E}$, yet both $\{2\}$ and $\{4\}$ (albeit for different reasons) make the same move out of $x:\{2\}$ compares $y$ to $z$, whereas $\{4\}$ compares y to $x$. Thus, $\{y, z\}$ is an ESS.

We first show that our axioms reduce to those proposed by Dutta \& Vohra (2017) if the list $F(x)$ contains for any $x$ at most two elements (including $(\emptyset, x)$ ), i.e. if $F$ corresponds to a (basic) expectation function.

Lemma 5.1. Let $F$ be an absorbing extended expectation function. If $F$ satisfies $\mathbf{I}$ then $F^{1}$ satisfies $\mathbf{I}$; if $F$ satisfies $\mathbf{M}$ then $F^{1}$ satisfies $\mathbf{M}^{\prime}$; if $F$ satisfies $\mathbf{E}$ and $k(x) \leq 2$ for all $x \in X$ then $F^{1}$ satisfies $\mathbf{E}$.

\footnotetext{
${ }^{4} F$ is uniquely defined as the effectivity correspondence $E$ does not allow any more moves. Throughout the chapter we shall specify $F$ only at those states $x \in X$ where $E(x, y) \neq \emptyset$ for some $y \neq x$, i.e. at those states for which non-trivial moves are allowed.
} 
Proof. Since $F$ is absorbing, $P_{F}(x)$ has a terminal node for each $x \in X$. Suppose $F$ satisfies $\mathbf{I}$, and let $x \in X$ be such that $x=f^{1}(x)$. If $y \neq x$ and $T \in E(x, y)$ then $u_{i}\left(x, F^{1}\right)=u_{i}\left(f^{1}(x), F^{1}\right) \geq u_{i}\left(y, F^{1}\right)$ for some $i \in T$ by $\mathbf{I}$. So $F^{1}$ satisfies I'.

Let $F$ satisfy M, let $x \in X$, and let $y \neq f^{1}(x)$ be such that $S^{1}(x) \in E(x, y)$. Then there is $i \in S^{1}(x)$ with $u_{i}\left(f^{1}(x), F^{1}\right) \geq u_{i}\left(y, F^{1}\right)$ by M. So, $F^{1}$ satisfies M'.

Let $F$ satisfy $\mathbf{E}$ and let $x \in X$. If $k(x)=1$, there is nothing to show. If $k(x)=2$ then $f^{2}(x)=x$ and $u_{i}\left(f^{1}(x), F^{1}\right)>u_{i}\left(f^{2}(x), F^{1}\right)=U_{i}(x)$ by $\mathbf{E}$. So, $F^{1}$ satisfies E'.

So, while an extended expectation function specifies much more than a basic expectation function, its first layer, namely $F^{1}$, is a basic expectation function; and this function inherits the properties I' and M' if $F$ satisfies I and M. On the other hand, E' will, in general, not be satisfied, but this is exactly what we aimed for in order to achieve full farsightedness. We close this section by briefly illustrating that the issues raised in Examples 5.1 and 5.2 can be resolved with this approach.

Example 5.4. Recall Example 5.1 and Figure 2(a). An extended expectation function that satisfies I must prescribe a move of $\{2\}$ from $x$ to $z$, irrespective of $\{1\}$ 's move at $x$. So, we either have $F(x)=(y,\{1\}),(z,\{2\}),(x, \emptyset))$ or $F(x)=((z,\{2\}),(x, \emptyset))$. An extended expectation function with $F(x)=((z,\{2\}),(y,\{1\}),(x, \emptyset))$, however, would violate $\boldsymbol{E}$, as in this case $\{1\}$ could maintain her payoff in $x$ by not moving (if she ever had the chance to move).

Recall Example 5.2 and Figure 2(b). An extended expectation function that satisfies all three axioms could have $F(w)=((x,\{1,2\}),(y,\{1\}),(z,\{2,3\}))$. $\boldsymbol{E}$ is not violated since any attempt of $\{2\}$ to boycott a move from $w$ to $x$ would ultimately result in $y$. Yet, $F(w)=((x,\{1,2\}),(z,\{2,3\}),(y,\{1\}))$ violates $\boldsymbol{E}$ as in this case 2 could beneficially veto $x$ and achieve outcome $z$.

The foregoing examples illustrate the crucial role of the order in which coalitions can move at a given state. We will shed more light on this order in Section 5.5 where we provide a non-cooperative foundation of rational extended expectation functions. At this moment we only note that it is intrinsic to the extended expectation function, and our axioms are independent of it.

\subsection{EXAMPLES}

In this section we will briefly illustrate how the ESS compares to other solutions that have been proposed in the literature and that are based on farsighted dominance relations. Let $x, y \in X$. State $y$ farsightedly dominates $x$ under the effectivity correspondence $E$, 
denoted as $y \succ^{f} x$, if there is a sequence $y^{0},\left(y^{1}, S^{1}\right), \ldots,\left(y^{m}, S^{m}\right)$, with $y^{0}=x$ and $y^{m}=y$, such that for all $k=1, \ldots, m$

$$
S^{k} \in E\left(y^{k-1}, y^{k}\right) \quad \text { and } \quad U_{i}(y)>U_{i}\left(y^{k-1}\right) \text { for all } i \in S^{k} .
$$

Chwe (1994) adapted the stability axioms of von Neumann \& Morgenstern (1944) by using this farsighted dominance relation:

FARSIGHTED INTERNAL STABILITY. A set $Y \subseteq X$ is farsightedly internally stable if there are no $x, y \in Y$ such that $y \succ^{f} x$,

FARSIGHTED EXTERNAL STABILITY. A set $Y \subseteq X$ is farsightedly externally stable if for every $x \in X \backslash Y$ there is $y \in Y$ such that $y \succ^{f} x$.

A set $Y \subseteq X$ is farsightedly stable if it is both farsightedly internally stable and farsightedly externally stable. A set $Y \subseteq X$ is consistent if for all $y \in Y$ and all $x \in X$ and all $S \in E(y, x)$ there is $z \in Y$ such that $z=x$ or $z \succ^{f} x$, and $u_{i}(z) \leq u_{i}(y)$ for some $i \in S$. Chwe (1994) shows that for any abstract game there is a unique largest ${ }^{5}$ consistent set (LCS). We shall use the remainder of this section to show that the ESS is different from the farsighted stable set, the largest consistent set, and the (strongly) rational expectation farsighted set.

Example 5.5. Consider the game given in Figure $4 .{ }^{6}$ The farsighted stable set contains states $y$ and $z$ as these are terminal nodes of the game from which no coalition can deviate. States $w$ and $x$ are not contained in the farsighted stable set as both are farsightedly dominated by $y$. The only rational extended expectation function is as follows: at state $w$ coalition $\{1\}$ is expected to stay in $w$ while in state $x$ coalition $\{2\}$ is expected to move to z. Thus, ESS, SREFS, and LCS are given by $\{w, y, z\}$. This set, however, violates farsighted internal stability.

Example 5.6. Consider the game depicted in Figure 5. We construct two different ESS's. First let $F^{1}(v)=(t,\{1\}), F^{2}(v)=(w,\{2\}), F^{1}(y)=(z,\{2\})$, and $F^{2}(y)=(x,\{1\})$. Then $F^{1}(w)=(y,\{2\})$ and $F^{1}(x)=(v,\{1\})$ by $\boldsymbol{I}$. Although $\{1\}$ 's move at $v$ leads to a lower payoff than 1 would obtain in $v$, the expectation of $\{2\}$ 's hypothetical future behavior makes this move beneficial. So, $F$ is a rational extended expectation function with $\mathcal{S}(F)=\{t, z\}$. This ESS, however, is neither a REFS nor externally stable as neither $v$ nor $y$ are farsightedly dominated by $t$ or $z$.

Let now $F^{1}(v)=(w,\{2\}), F^{2}(v)=(v, \emptyset), F^{1}(y)=(x,\{1\})$, and $F^{2}(y)=(y, \emptyset)$. Then $F$ with $F^{1}(s)=(s, \emptyset)$ for all $s \neq v, y$ is another rational extended expectation

\footnotetext{
${ }^{5}$ With respect to set inclusion.

${ }^{6}$ The example is borrowed from Dutta \& Vohra (2017).
} 
Figure 4: Violation of internal farsighted stability.

$$
\begin{gathered}
w(1,1) \stackrel{\{1\}}{\longrightarrow} x(0,0) \stackrel{\{2\}}{\longrightarrow} y(10,10) \\
\qquad\{2\} \\
z(0,20)
\end{gathered}
$$

Figure 5: Violation of farsighted external stability.

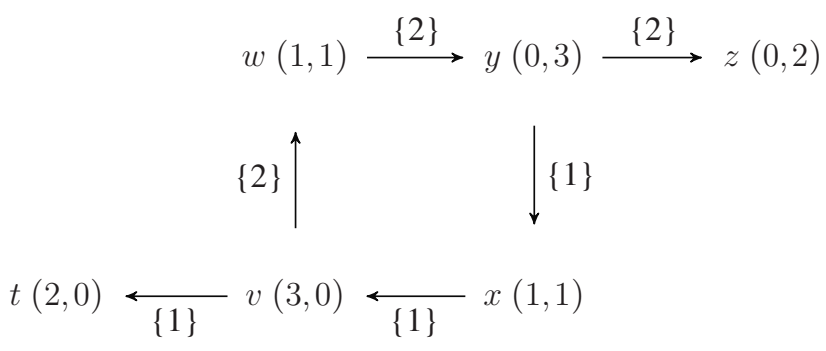

function with $\mathcal{S}(F)=\{t, w, x, z\}$. In this case there is in every node at most one coalitions that would move out of it, so the set of terminal nodes is also a REFS by Lemma 5.1.

Example 5.7. Consider the game depicted in Figure 6. By I coalition $\{2\}$ must move from $x$ to $z$. Hence, one rational extended expectation function is given by $F^{1}(w)=(x,\{1\})$, $F^{1}(x)=(y,\{1\})$ and $F^{2}(x)(z,\{2\})$. In this case $\mathcal{S}(F)=\{y, z\}$. However, $\{1\}$ 's move at $x$ violates $\boldsymbol{E}$ ', and $\boldsymbol{I}$ ' requires $\{2\}$ to move from $x$ to $z$. So, at state $w$ coalition $\{1\}$ is expected to stay in $w$ by $\boldsymbol{E}^{\prime}$. Therefore, the SREFS is $\{w, y, z\}$. This is identical to the farsighted stable set as well as the LCS and could be supported as an ESS if $\{2\}$ was allowed to move before $\{1\}$ at $x$.

We have seen that our solution is different from those proposed in the previous literature. So, what do we gain from a new solution? Our first answer, which we have elaborated thus far, is that the ESS is the only solution that respects full farsightedness of players. We will give our second answer in the next section, where we show that rational extended 
Figure 6: ESS vs. REFS (SREFS).

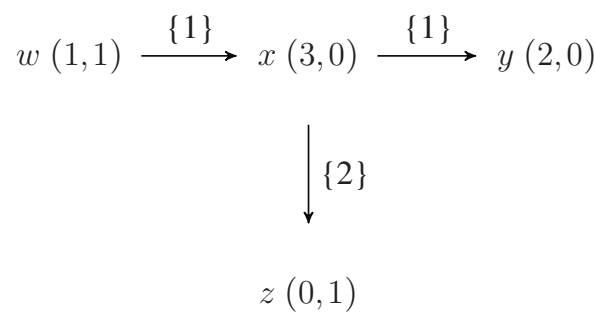

expectation functions are exactly those extended expectation functions that emerge from a strategy profile in the original game which is stable with respect to coalition deviations.

\subsection{NON-COOPERATIVE FOUNDATION}

Let $G$ be an abstract game and let $\emptyset \neq S \subseteq N$. A strategy of coalition $S$ is a map $\sigma_{S}: X \rightarrow X$ with $S \in E\left(S, \sigma_{S}(x)\right)$ for all $x \in X$. Denote the set of $S$ 's strategies by $\Sigma_{S}$. Let $\sigma^{0}: X \rightarrow X$ be defined as $\sigma^{0}(x)=x$ for all $x \in X$ and observe that $\sigma^{0} \in \Sigma_{S}$ for all $\emptyset \neq S \subseteq N$. To avoid trivialities and technical issues let $\Sigma_{\emptyset}=\left\{\sigma^{0}\right\}$. A strategy profile is a vector $\left(\sigma_{S}\right)_{S \subseteq N} \in \times_{S \subseteq N} \Sigma_{S}$ of strategies, and the set of all strategy profiles is denoted by $\Sigma$.

\subsubsection{Strategies and extended expectation functions}

A strategy profile $\sigma \in \Sigma$ induces some expectations among players, namely that each coalition moves according to their strategy. However, this expectation does not uniquely define an extended expectation function as at any state several coalitions might wish to move. In order to associate a unique extended expectation function $F_{\sigma}$ to a strategy profile $\sigma$, we suppose that at each state $x$ coalitions are allowed to move according to a linear order $\succeq^{x}$ such that $S \succeq^{x} \emptyset$ for all $S \subseteq N$, where $S \succeq^{x} T$ denotes that $S$ moves before $T$ at $x$. We then define $F_{\sigma}(x)=\left(F_{\sigma}^{1}(x), \ldots, F_{\sigma}^{k(x)}(x)\right)$ such that

1. $T \in\left\{S^{1}(x), \ldots, S^{k(x)-1}(x)\right\}$ if and only if $\sigma_{T}(x) \neq x$,

2. $S^{l}(x) \succeq^{x} S^{l+1}(x)$ for all $l=1, \ldots, k(x)-1$,

3. $f^{l}(x)=\sigma_{S^{l}(x)}(x)$ for all $l=1, \ldots, k(x)-1$, 
Figure 7: Better responses

$$
x(1,1) \stackrel{\{1,2\}}{\longrightarrow} y(1,2) \stackrel{\{1,2\}}{\longrightarrow} z(2,2)
$$

4. $S^{k(x)}(x)=\emptyset$ and $f^{k(x)}(x)=x$.

This construction is unique for any $\sigma \in \Sigma$ and any order profile $\succeq=\left(\succeq^{x}\right)_{x \in X}$. In the sequel we shall write $F_{\sigma}$, bearing in mind that $F_{\sigma}$ depends on $\succeq$ as well. The order profile $\succeq$ shall be common knowledge. While it is clear that $F_{\sigma}$ is an extended expectation function, $F_{\sigma}$ need neither be absorbing nor satisfy any of our axioms.

\subsubsection{Better responses}

We shall now focus on strategy profiles. In order to keep notation simple, we shall write $u_{i}(\sigma, x)$ for $u_{i}\left(x, F_{\sigma}\right)$. Let $\emptyset \neq S \subseteq N$, let $\sigma_{S}, \sigma_{S}^{\prime} \in \Sigma_{S}$ and $\sigma_{-S} \in \Sigma_{-S}=\times_{T \neq S} \Sigma_{T}$. Strategy $\sigma_{S}$ is a better response against $\sigma_{-S}$ than $\sigma_{S}^{\prime}$ at $x$ if

$$
u_{i}\left(\left(\sigma_{S}, \sigma_{-S}\right), x\right)>u_{i}\left(\left(\sigma_{S}^{\prime}, \sigma_{-S}\right), x\right)
$$

for all $i \in S$. Say that $\sigma_{S}$ is a better response against $\sigma_{-S}$ than $\sigma_{S}^{\prime}$ if it is a better response at all $x \in X$ with $\sigma_{S}(x) \neq \sigma_{S}^{\prime}(x)$. The latter requirement is similar to the definition of coalition deviations in Kimya (2018). While it seems strong at first sight, the following example illustrates its importance.

Example 5.8. Consider the game depicted in Figure 7 and strategy profile $\sigma$ with $\sigma_{S}=\sigma^{0}$ for all $S \subseteq N$. At $x$ coalition $\{1,2\}$ could strictly improve by switching to $\sigma^{\prime}$ with $\sigma_{\{1,2\}}^{\prime}(x)=y$ and $\sigma_{\{1,2\}}^{\prime}(y)=z$. However, if we consider only that part of the game which starts at $y$, we would not claim that the coalition's switching from $y$ to $z$ is a profitable deviation, as 2 does not profit from it. So, $\sigma_{\{1,2\}}^{\prime}$ is not a better response against $\sigma_{-\{1,2\}}$ than $\sigma_{\{1,2\}}$ albeit being a better response at $x$.

One could argue that in Example 5.8 there is no reason for player 2 not to join $\{1\}$ in implementing $\sigma^{\prime}$. And one could define better responses slightly differently in order to avoid such an argument, namely by requiring that for all $x \in X$ the weak version of Inequality (10) is satisfied for all $i \in S$ and its strict version is satisfied for some $i \in S$. But we want to make our solution comparable to other notions of dominance, and they are typically defined in terms of strict inequalities for all members of the deviating coalition. Nevertheless the proof of the next lemma would also hold for the alternative definition. 
Figure 8: Better responses and objected strategies

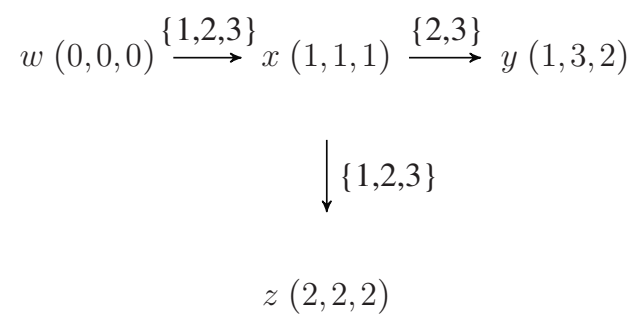

Lemma 5.2 (One-shot deviation principle). Let $\emptyset \neq S \subseteq N$, let $\sigma_{S}, \sigma_{S}^{\prime} \in \Sigma_{S}$ and $\sigma_{-S} \in \Sigma_{-S}$. If $\sigma_{S}$ is a better response against $\sigma_{-S}$ than $\sigma_{S}^{\prime}$ then there are $x^{*} \in X$ and $\sigma_{S}^{*} \in \Sigma_{S}$ such that $\sigma_{S}^{*}$ is a better response against $\sigma_{-S}$ than $\sigma_{S}^{\prime}$ as well, and $\sigma_{S}^{*}(x)=\sigma_{S}^{\prime}(x)$ for all $x \neq x^{*}$.

Proof. Let $\sigma_{S}, \sigma_{S}^{\prime} \in \Sigma_{S}$ be as defined and let $x \in X$ be such that $u_{i}\left(\left(\sigma_{S}, \sigma_{-S}\right), x\right)>$ $u_{i}\left(\left(\sigma_{S}^{\prime}, \sigma_{-S}\right), x\right)$ for all $i \in S$. Such $x \in X$ exists by the definition of $\sigma_{S}$, and $u_{i}\left(\left(\sigma_{S}, \sigma_{-S}\right), x\right)>-\infty$ for all $i \in S$. Let $\left(x^{1}, \ldots, x^{m}\right)$ be the path that is implemented by $\left(\sigma_{S}, \sigma_{-S}\right)$ starting at $x$; that is, $x^{1}=x, x^{m}=t\left(P_{F_{\left(\sigma_{S}, \sigma_{-}\right)}}(x)\right)$, with $F_{\left(\sigma_{S}, \sigma_{-S}\right)}$ as defined in Subsection 5.5.1. (This path is finite as $u_{i}\left(\left(\sigma_{S}, \sigma_{-S}\right), x\right)>-\infty$ for all $i \in S$.) Let $h \in\{1, \ldots, m\}$ be such that $\sigma_{S}\left(x^{h}\right) \neq \sigma_{S}^{\prime}\left(x^{h}\right)$ and $\sigma_{S}\left(x^{l}\right)=\sigma_{S}^{\prime}\left(x^{l}\right)$ for $l>h$. Let $x^{*}=x^{h}$. As $\sigma_{S}$ is a better response against $\sigma_{-S}$ than $\sigma_{S}^{\prime}$ at all $x^{\prime}$ with $\sigma_{S}\left(x^{\prime}\right) \neq \sigma_{S}^{\prime}\left(x^{\prime}\right)$, one has in particular that $\sigma_{S}$ is a better response than $\sigma_{S}^{\prime}$ at $x^{*}$. Define now $\sigma_{S}^{*}$ by $\sigma_{S}^{*}(x)=\sigma_{S}^{\prime}(x)$ for all $x \neq x^{*}$ and $\sigma_{S}^{*}\left(x^{*}\right)=\sigma_{S}\left(x^{*}\right)$. Then $\sigma_{S}^{*}$ is a better response than $\sigma_{S}^{\prime}$

Example 5.9. Let $N=\{1,2,3\}, X=\{w, x, y, z\}$, and for all $x \in X$ let $\succeq^{x}$ be such that $S \succeq^{x} T$ whenever $T \subseteq S$. Figure 8 depicts an effectivity correspondence and payoffs for all players. Coalition $\{1,2,3\}$ is always the first coalition to move, and irrespective of the strategy of coalition $\{2,3\}$ there is no better response than their moving from $w$ to $x$ and from $x$ to $z$.

Observe that in the previous example $\{1,2,3\}$ is not effective for any moves out of $y$ and $z$ according to the effectivity correspondence in Figure 8. Hence, the behavior at $w$ and $y$ fully determines their strategy. In order to keep notation simple, we will leave out all trivial parts of strategies in the remainder of the chapter. 


\subsubsection{Objections}

We have carefully avoided the term "best response" thus far as the strategy in Example 5.9 has a severe caveat: player 2 has no reason to support the joint move from $x$ to $z$. By vetoing such a decision, ${ }^{7}$ the order $\succeq$ would guarantee that $\{2,3\}$ would make their move next, achieving $y$ with payoff 3 for player 2 . In order to formalize this idea, we need a little more notation. For $\emptyset \neq S \subseteq N, \sigma_{S} \in \Sigma_{S}$, and $x \in X$ define $\sigma_{S}^{0, x}$ by

$$
\sigma_{S}^{0, x}(y)= \begin{cases}\sigma_{S}(y) & \text { if } y \neq x \\ x & \text { if } y=x\end{cases}
$$

Note that $\sigma_{S}^{0, x} \in \Sigma_{S}$ for all $\sigma_{S} \in \Sigma_{S}$ since $S \in E(x, x)$ for all $x \in X$. The only (potential) difference between $\sigma_{S}^{0, x}$ and $\sigma_{S}$ is that the former will map $x$ on itself. We say that agent $i \in S$ objects $\sigma_{S}$ at $x$ given $\sigma_{-S}$ if $\sigma_{S}(x) \neq x, \sigma_{T}(x)=x$ for all $T \succeq^{x} S$, and

$$
u_{i}\left(\left(\sigma_{S}^{0, x}, \sigma_{-S}\right), x\right) \geq u_{i}\left(\left(\sigma_{S}, \sigma_{-S}\right), x\right) .
$$

The idea behind objections is that a player will not join $S$ in implementing $\sigma_{S}$ if she cannot profit by doing so. We say that agent $i \in S$ objects $\sigma_{S}$ if there is some $x \in X$ such that $i$ objects $\sigma_{S}$ at $x$. Say $\sigma_{S}$ is objected if it is objected by some $i \in S$.

Example 5.10. Given the order in Example 5.9 the strategy of coalition $\{1,2,3\}$ is objected at $x$ by players 2 and 3 .

In the definition of an objected strategy $\sigma_{S}$ given $\sigma_{-S}$ at $x$ we have imposed the condition that $\sigma_{T}(x)=x$ for all $T \succeq^{x} S$. The reason is that if a coalition $S$ were allowed to move at $x$ only after some other coalition $T$ has already moved out of $x$, then $S$ 's behavior at $x$ is (trivially) payoff-irrelevant. Nevertheless, $S$ might strictly benefit from $\sigma_{S}$ at $x$ if they had a chance to actually implement it. We have to carefully distinguish between strategies that are not profitable at $x$ (compared to $\sigma_{S}^{0}$ ) because their implementation would not be profitable, and those that cannot be implemented because of the order $\succeq^{x}$. The former strategies are objected, the latter will be investigated more closely in Subsection 5.5.4.

We next formulate, similarly to Lemma 5.2, a one-shot deviation principle for unobjected better responses.

Lemma 5.3. Let $\emptyset \neq S \subseteq N$, let $\sigma_{S}, \sigma_{S}^{\prime} \in \Sigma_{S}$ and $\sigma_{-S} \in \Sigma_{-S}$ such that $\sigma_{S}^{\prime}$ is unobjected given $\sigma_{-S}$. If $\sigma_{S}$ is an unobjected better response against $\sigma_{-S}$ than $\sigma_{S}^{\prime}$ then there are

\footnotetext{
${ }^{7}$ We make the implicit assumption here that a coalition $S$ can implement a move from $x$ to $y \neq x$ only if all members unanimously agree on it. This is a standard assumption in the literature on dominance relations, and the consequence is that any member of $S$ can veto any move out of $x$.
} 
$x^{*} \in X$ and $\sigma_{S}^{*} \in \Sigma_{S}$ such that $\sigma_{S}^{*}$ is an unobjected better response against $\sigma_{-S}$ than $\sigma_{S}^{\prime}$ as well, and $\sigma_{S}^{*}(x)=\sigma_{S}^{\prime}(x)$ for all $x \neq x^{*}$.

Proof. Construct $x^{*}$ and $\sigma_{S}^{*}$ as in the proof of Lemma 5.2. For each $x \in X$ it holds that either

$$
t\left(P_{F_{\left(\sigma_{S}^{*}, \sigma_{-S}\right)}}(x)\right)=t\left(P_{F_{\left(\sigma_{S}^{\prime}, \sigma_{-S}\right)}}(x)\right)
$$

or

$$
t\left(P_{F_{\left(\sigma_{S}^{*}, \sigma_{-S}\right)}}(x)\right)=t\left(P_{F_{\left(\sigma_{S}, \sigma_{-S}\right)}}(x)\right)
$$

and, hence, $u\left(\left(\sigma_{S}^{*}, \sigma_{-S}\right), x\right)=u\left(\left(\sigma_{S}^{\prime}, \sigma_{-S}\right), x\right)$ or $u\left(\left(\sigma_{S}^{*}, \sigma_{-S}\right), x\right)=u\left(\left(\sigma_{S}, \sigma_{-S}\right), x\right)$. In the former case $\sigma_{S}^{*}$ is unobjected at $x$ as $\sigma_{S}^{\prime}$ is; in the latter case $\sigma_{S}^{*}$ is unobjected at $x$ as $\sigma_{S}$ is.

Note that the construction in Lemma 5.3 relies on $\sigma_{S}^{\prime}$ 's being unobjected. In particular, it is, in general, not true that for all $\sigma_{S}^{\prime}$ for which an unobjected better response exists, there is an unobjected better response that differs from $\sigma_{S}^{\prime}$ at only one state $x^{*} \in X$.

\subsubsection{Incredible threats}

As there is a fixed order in which coalitions are allowed to move, there might be a state $x$ and a coalition $S$, such that $S$ will not have the opportunity to move at $x$ given strategy vector $\sigma_{-S}$. Coalition $S$ 's action at $x$ will thus never be implemented and does not affect payoffs. The following example illustrates the resulting issues.

Example 5.11. Let $N=\{1,2\}, X=\{x, y, z\}$ and $\{1\} \succeq^{x}\{2\}$ for all $x \in X$, and consider the effectivity correspondence and payoffs depicted in Figure 9. Let $\sigma_{\{2\}}(x)=z$. In this case, $\{1\}$ should play $\sigma_{\{1\}}(x)=y$ to secure at least some positive payoff. And if she does so, $\{2\}$ 's action at $x$ is payoff-irrelevant, as she will never move anyway. So, there is no better response for $\{2\}$ against $\sigma_{-\{2\}}$ than $\sigma_{\{2\}}$.

The reader will have realized that this example is closely related to the problem of subgame perfect equilibria. The issue, of course, is that in an abstract game there is no initial node, there is no order in which the game is being played, and cycles among states are possible. So, in order to capture the idea of a subgame in this context, one has to work a little. For $x \in X$ and $\emptyset \neq S \subseteq N$ let

$$
E^{S, x}\left(x^{\prime}, y\right)= \begin{cases}\left\{T \in E\left(x^{\prime}, y\right): S \succeq^{x} T\right\} & \text { if } x^{\prime}=x, \\ E\left(x^{\prime}, y\right) & \text { otherwise. }\end{cases}
$$


Figure 9: What should 1 do if 2 threatens to move to $z$ ?

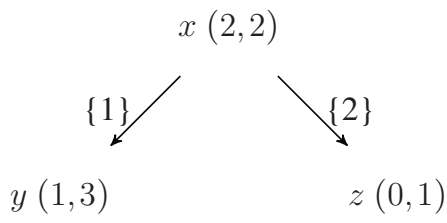

The effectivity correspondences $E$ and $E^{S, x}$ are almost identical, the only difference being that according to $E^{S, x}$ those coalitions that are allowed to move prior to $S$ at $x$ are not effective for any move out of $x$. As they have to stay in $x, S$ can actually implement their move. The corresponding abstract game is defined as $G^{S, x}=\left(N, X, E^{S, x},\left(U_{i}(.)\right)_{i \in N}\right)$. As the effectivity correspondence $E^{S, x}$ is different from $E$, the strategy space in $G^{S, x}$ is different from the one in $G$ as well: denote by $\Sigma^{S, x}$ the strategy space for this abstract game, and observe that $\Sigma_{S}^{S, x}=\Sigma_{S}$, that is, $S$ has the same strategy space as before. For $\sigma \in \Sigma$ let the strategy vector $\sigma^{S, x} \in \Sigma^{S, x}$ be defined by

$$
\sigma_{T}^{S, x}(y)= \begin{cases}y & \text { if } y=x \text { and } T \succeq^{x} S, \\ \sigma_{T}(y) & \text { otherwise. }\end{cases}
$$

That is, the only change between $\sigma_{T}$ and $\sigma_{T}^{S, x}$ lies in the behavior of $T$ at $x$ if $T$ is allowed to move before $S$ at $x$ : in this case $T$ does not move out of $x$. Again, it is easy to see that $\sigma_{S}^{S, x}=\sigma_{S}$ for all $S \subseteq N$ and all $x \in X$.

In some sense we can interpret $G^{S, x}$ as a subgame of the abstract game $G$. The game $G^{S, x}$ allows $S$ 's move at $x$ to be implemented, as $S$ is the first coalition to move. This construction allows us to prescribe "sensible" behavior even in states where a coalition does, originally, not move. In particular, we will later impose the condition that $\sigma_{S}$ is not only a "good" strategy against $\sigma_{-S}$, but that also against $\sigma_{-S}^{S, x}$ at all $x$. This roughly corresponds to the idea of subgame perfection for coalitions.

It is worth mentioning, though, that state $x$ might be reached more than once as a strategy profile $\sigma$ might induce cycles - a phenomenon that cannot appear in a standard dynamic game. The definition above ensures that even if $x$ is reached a second time in $G^{S, x}$, it is again $S$ who has to move first. That means that $S$ can not throw the ball back to a coalition $T$ that would, in the original game $G$, move prior to $S$ at $x$.

Example 5.12. Recall Example 5.11 and Figure 9. In the game $G^{\{2\}, x}$ strategy $\sigma_{\{2\}}^{0}$ is a better response than the (unique) strategy $\sigma_{\{2\}}$ with $\sigma_{\{2\}}(x)=z$. 
Figure 10: What should $\{1,2\}$ do?

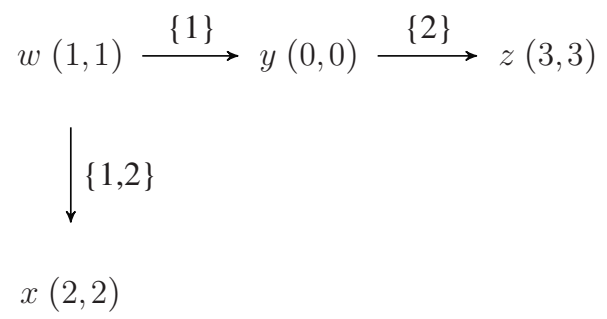

\subsubsection{Best responses}

Defining best responses for coalitions entails the difficulty that various subcoalitions might implement better actions in various states. The next example illustrates this point.

Example 5.13. Let $N=\{1,2\}, X=\{w, x, y, z\}$, for all $x \in X$ let $\succeq^{x}$ be given by $S \succeq^{x} T$ if $T \subseteq S$, and consider the effectivity correspondence and payoffs depicted in Figure 10. Then for coalition $\{1,2\}$ it is optimal to remain in $w$ such that $\{1\}$ can move to $y$ and $\{2\}$ can move to $z$.

We are now ready to define best responses. During the preceding discussions we have identified four requirements that one would expect: (i) a best response for a coalition $S$ must be a vector of strategies, one for each subcoalition of $S$ (see Example 5.13); (ii) the strategy must be unobjected, even in those states where $S$ will never be allowed to actually execute their strategy (see Examples 5.11 and 5.12); (iii) there must not be any (unobjected) better response (see Example 5.9); and (iv) it must be optimal for each subcoalition of $S$ as well (see Example 5.10). The following definition puts these requirements together.

Definition 5.2. Let $\emptyset \neq S \subseteq N$ and let the order profile $\succeq$ be given. A strategy vector $\sigma_{P(S)}$ is a best response against $\sigma_{-P(S)}$ if

1. for each $x \in X$ strategy $\sigma_{S}$ is unobjected against $\sigma_{-S}^{S, x}$ at $x$ in the game $G^{S, x}$,

2. for each $x \in X$ and each $\tau_{S} \in \Sigma_{S}$ that is a better response than $\sigma_{S}$ against $\sigma_{-S}^{S, x}$ at $x$ in the game $G^{S, x}$ there is $i \in S$ who objects $\tau_{S}$,

3. $\sigma_{T}$ is a best response for all $\emptyset \neq T \subsetneq S$.

The following example illustrates how best responses might depend on $\succeq$. 
Example 5.14. Recall Example 5.13 and Figure 10. If $\{1\}$ moves before $\{1,2\}$ at $w$ then $\sigma_{\{1,2\}}(w)=x$ as a tribute to Condition 2 in Definition 5.2.

\subsubsection{Equilibrium}

Definition 5.3. Let $\succeq$ be an order profile. A strategy profile $\sigma$ is an equilibrium (with respect to $\succeq)$ if for all $\emptyset \neq S \subseteq N$ the vector $\sigma_{P(S)}$ is a best response against $\sigma_{-P(S)}$.

Recall that the definition of best responses is recursive: a coalition's best response contains best responses for each subcoalition. So, we obtain the following characterization of equilibria which is mainly stated for later reference and does not require a proof.

Proposition 5.1. The following are equivalent:

1. $\sigma$ is an equilibrium.

2. $\sigma$ is a best response for $N$.

3. for each $x \in X$ and each $\emptyset \neq S \subseteq N$ strategy $\sigma_{S}$ is unobjected against $\sigma_{-S}^{S, x}$ at $x$ in the game $G^{S, x}$, and for each $\tau_{S} \in \Sigma_{S}$ that is a better response against $\sigma_{-S}^{S, x}$ at $x$ than $\sigma_{S}$ in the game $G^{S, x}$ there is $i \in S$ who objects $\tau_{S}$.

As best responses depend on $\succeq$, so do equilibria and the outcome of the game. We now come to our main result that connects equilibria and rational expectation functions.

Theorem 5.1. An extended expectation function $F$ is rational if and only if there are an order profile $\succeq$ and an equilibrium $\sigma$ with respect to $\succeq$ such that $F=F_{\sigma}$.

Proof. Let $\succeq$ be an order profile, let $\sigma$ be an equilibrium with respect to $\succeq$, and let $F=F_{\sigma}$. We prove that $F$ satisfies I. Assume this were not the case. Then there are $x \in X$ and $T \in 2^{N} \backslash\left\{S^{1}(x), \ldots, S^{k(x)}(x)\right\}$ such that for each $l=1, \ldots, k(x)$ there is $y \in X$ with $T \in E(x, y)$ and $u_{i}(y, F)>u_{i}\left(f^{l}(x), F\right)$ for all $i \in T$. Let $T$ and $x$ be as described, let $l \in\{1, \ldots, k(x)\}$ be the minimal number with $T \succeq^{x} S^{l}(x)$ (this is well-defined as $\left.T \succeq^{x} \emptyset=S^{k(x)}(x)\right)$, and let $y$ be such that $u_{i}(y, F)>u_{i}\left(f^{l}(x), F\right)$ for all $i \in T$. Consider the game $G^{T, x}$, recall that $\Sigma_{T}^{T, x}=\Sigma_{T}$, and let $\sigma_{T}^{\prime} \in \Sigma_{T}$ be defined by

$$
\sigma_{T}^{\prime}\left(x^{\prime}\right)= \begin{cases}\sigma_{T}\left(x^{\prime}\right) & \text { if } x^{\prime} \neq x \\ y & \text { otherwise }\end{cases}
$$

Clearly, $\sigma_{T}^{\prime}$ is a better response against $\sigma_{-T}^{T, x}$ at $x$ than $\sigma_{T}$. As $\sigma$ is an equilibrium, $\sigma_{T}^{\prime}$ must be objected by Proposition 5.1. As $\sigma_{T}^{\prime}$ and $\sigma_{T}$ coincide everywhere except $x$, and since 
$\sigma_{T}$ is not objected, $\sigma_{T}^{\prime}$ can only be objected in some $z \in X$ with $x \in P_{F}(z)$. For such $z$ it holds that $u_{i}\left(\left(\sigma_{T}^{\prime}, \sigma_{-T}^{T, x}\right), z\right)=u_{i}(y, F)$ and $u_{i}\left(\left(\sigma_{T}, \sigma_{-T}^{T, x}\right), z\right)=u_{i}\left(\left(\sigma_{T}, \sigma_{-T}^{T, x}\right), x\right)=$ $u_{i}\left(f^{l}(x), F\right)$ by construction. Since $\sigma_{T}^{\prime}$ is objected at $z$, and $\sigma_{T}$ is not, there is $i \in T$ with

$$
\begin{aligned}
u_{i}\left(\left(\sigma_{T}, \sigma_{-T}^{T, x}\right), x\right) & =u_{i}\left(f^{l}(x), F\right)<u_{i}(y, F) \\
& =u_{i}\left(\left(\sigma_{T}, \sigma_{-T}^{T, x}\right), y\right)=u_{i}\left(\left(\sigma_{T}^{\prime}, \sigma_{-T}^{T, x}\right), x\right) \\
& =u_{i}\left(\left(\sigma_{T}^{\prime}, \sigma_{-T}^{T, x}\right), z\right) \leq u_{i}\left(\left(\sigma_{T}^{0, z}, \sigma_{-T}^{T, x}\right), z\right) \\
& <u_{i}\left(\left(\sigma_{T}, \sigma_{-T}^{T, x}\right), z\right)=u_{i}\left(\left(\sigma_{T}, \sigma_{-T}^{T, x}\right), x\right),
\end{aligned}
$$

where the first two (in)equalities have been elaborated before, and the remaining ones (in that order) hold because of the definition of $F$, the construction of $\sigma_{T}^{\prime}$, the definition of $z, \sigma_{T}^{\prime}$ 's being objected at $z, \sigma_{T}$ 's being unobjected at $z$, and again the definition of $z$. But the overall inequality is impossible. Hence, such $y$ cannot exist. That means that $F$ satisfies I. We next prove that $F$ satisfies E. Assume this were not the case. Then there are $x \in X$ and $l \leq k(x)-1$ such that $u_{i}\left(f^{l+1}(x), F\right) \geq u_{i}\left(f^{l}(x), F\right)$ for some $i \in S^{l}(x)$. By definition

$$
u_{i}\left(f^{l}(x), F\right)=u_{i}\left(\left(\sigma_{S^{l}(x)}, \sigma_{-S^{l}(x)}^{S^{l}(x), x}\right), x\right)
$$

and

$$
u_{i}\left(f^{l+1}(x), F\right)=u_{i}\left(\left(\sigma_{S^{l}(x)}^{0, x}, \sigma_{-S^{l}(x)}^{S^{l}(x), x}\right), x\right)
$$

Hence,

$$
\begin{aligned}
u_{i}\left(\left(\sigma_{S^{l}(x)}^{0, x}, \sigma_{-S^{l}(x)}^{S^{l}(x), x}\right), x\right) & =u_{i}\left(f^{l+1}(x), F\right) \\
& \geq u_{i}\left(f^{l}(x), F\right)=u_{i}\left(\left(\sigma_{S^{l}(x)}, \sigma_{-S^{l}(x)}^{S^{l}(x), x}\right), x\right)
\end{aligned}
$$

which means that $\sigma_{S^{l}(x)}$ is objected at $x$ by $i$. This contradicts $\sigma$ 's being an equilibrium. So, $F$ satisfies $\mathbf{E}$. 
We prove that $F$ satisfies M. Assume this were not the case. Then there are $x \in X$, $l \in\{1, \ldots, k(x)-1\}$, and $y \in X$ such that $S^{l}(x) \in E(x, y), f^{l}(x) \neq y$, and $u_{i}(y, F)>$ $u_{i}\left(f^{l}(x), F\right)$ for all $i \in S^{l}(x)$. Define $\sigma_{S^{l}(x)}^{\prime}$ by

$$
\sigma_{S^{l}(x)}^{\prime}\left(x^{\prime}\right)= \begin{cases}\sigma_{S^{l}(x)}\left(x^{\prime}\right) & \text { if } x^{\prime} \neq x \\ y & \text { if } x^{\prime}=x .\end{cases}
$$

Clearly, $\sigma_{S^{l}(x)}^{\prime}$ is a better response against $\sigma_{-S^{l}(x)}^{S^{l}(x), x}$ than $\sigma_{S^{l}(x)}$. Hence, as $\sigma$ is an equilibrium, $\sigma_{S^{l}(x)}^{\prime}$ must be objected. As $\sigma_{S^{l}(x)}^{\prime}$ and $\sigma_{S^{l}(x)}$ coincide everywhere except $x$, and since $\sigma_{S^{l}(x)}$ is not objected, $\sigma_{S^{l}(x)}^{\prime}$ can only be objected at some $z \in X$ with $x \in P_{F}(z)$. For such $z$ it holds that

$$
u_{i}\left(\left(\sigma_{S^{l}(x)}, \sigma_{-S^{l}(x)}^{S^{l}(x), x}\right), z\right)=u_{i}\left(\left(\sigma_{S^{l}(x)}, \sigma_{-S^{l}(x)}^{S^{l}(x), x}\right), x\right)=u_{i}\left(f^{l}(x), F\right)
$$

and

$$
u_{i}\left(\left(\sigma_{S^{l}(x)}^{\prime}, \sigma_{-S^{l}(x)}^{S^{l}(x), x}\right), z\right)=u_{i}(y, F) .
$$

Since $\sigma_{S^{l}(x)}^{\prime}$ is objected at $z$, and $\sigma_{S^{l}(x)}$ is not, there is $i \in S^{l}(x)$ with

$$
\begin{aligned}
u_{i}\left(\left(\sigma_{S^{l}(x)}, \sigma_{-S^{l}(x)}^{S^{l}(x), x}\right), x\right) & =u_{i}\left(f^{l}(x), F\right)<u_{i}(y, F) \\
& =u_{i}\left(\left(\sigma_{S^{l}(x)}^{\prime}, \sigma_{-S^{l}(x)}^{S^{l}(x), x}\right), z\right) \leq u_{i}\left(\left(\sigma_{S^{l}(x)}^{0, z}, \sigma_{-S^{l}(x)}^{S^{l}(x), x}\right), z\right) \\
& <u_{i}\left(\left(\sigma_{S^{l}(x)}, \sigma_{-S^{l}(x)}^{S^{l}(x), x}\right), z\right)=u_{i}\left(\left(\begin{array}{c}
\sigma_{S^{l}(x)}, \sigma_{-S^{l}(x)}^{S^{l}(x), x} \\
S^{l}
\end{array}\right), x\right),
\end{aligned}
$$

where the weak inequality follows from $\sigma_{S^{l}(x)}^{\prime}$ 's being objected at $z$, and the last strict inequality follows from $\sigma_{S^{l}(x)}$ 's not being objected. But the overall inequality is impossible. This proves that $F$ satisfies $\mathbf{I}, \mathbf{E}$, and $\mathbf{M}$. So, $F$ is rational.

Let now $F$ be a rational extended expectation function. Define $\sigma$ by

$$
\sigma_{S}(x)= \begin{cases}f^{l}(x) & \text { if } S=S^{l}(x) \text { for some } l=1, \ldots, k(x) \\ x & \text { otherwise. }\end{cases}
$$

For $x \in X$ define $\succeq^{x}$ such that

1. $S \succeq^{x} \emptyset$ for all $S \subseteq N$,

2. $S^{l}(x) \succeq^{x} S^{l+1}(x)$ for all $l=1, \ldots, k(x)-1$, 
3. for $S \in 2^{N} \backslash\left\{S^{1}(x), \ldots, S^{k}(x)\right\}$ it holds that $S^{l-1}(x) \succeq^{x} S \succeq^{x} S^{l}(x)$ where $l$ is the minimal number such that for all $y \in X$ there is $i \in S$ with $u_{i}\left(f^{l}(x), F\right) \geq u_{i}(y, F)$. (If $l=1$ let $S \succeq S^{1}(x)$.)

Since $F$ satisfies $\mathbf{I}$, such $\succeq^{x}$ exists for all $x \in X$. We show that $\sigma$ is an equilibrium with respect to $\succeq$, i.e. that $\sigma_{P(S)}$ is a best response against $\sigma_{-P(S)}$ for all $S \subseteq N$. Let $\emptyset \neq S \subseteq N$. By Proposition 5.1 it is sufficient to show that $\sigma_{S}$ is unobjected against $\sigma_{-S}^{S, x}$ in the game $G^{S, x}$ for all $x \in X$, and that each $\tau_{S} \in \Sigma_{S}$ that is a better response against $\sigma_{-S}^{S, x}$ than $\sigma_{S}$ in the game $G^{S, x}$ is objected. We first show that $\sigma_{S}$ is unobjected for all $x \in X$. If $x$ is such that $S \neq S^{l}(x)$ for all $l=1, \ldots, k(x)$, there is nothing to show as $\sigma_{S}(x)=x=\sigma_{S}^{0}(x)$. If $S=S^{l}(x)$ for some $l$ then $\sigma_{S}(x)=f^{l}(x)$, and by $\mathbf{E}$ it holds that

$$
u_{i}\left(\left(\sigma_{S}, \sigma_{-S}^{S, x}\right), x\right)=u_{i}\left(f^{l}(x), F\right)>u_{i}\left(f^{l+1}(x), F\right)=u_{i}\left(\left(\sigma_{S}^{0, x}, \sigma_{-S}^{S, x}\right), x\right)
$$

for all $i \in S$. Hence, $\sigma_{S}$ is not objected at any $x \in X$.

Next we show that there is no unobjected better response than $\sigma_{S}$. Assume on the contrary that there are $x \in X, \emptyset \neq S \subseteq N$, and $\sigma_{S}^{\prime} \in \Sigma_{S}$ such that $\sigma_{S}^{\prime}$ is an unobjected better response against $\sigma_{-S}^{S, x}$ in $G^{S, x}$. As $\sigma_{S}$ is unobjected, it is by Lemma 5.3 no loss of generality to assume that there is $x^{*} \in X$ such that $\sigma_{S}^{\prime}(x)=\sigma_{S}(x)$ for all $x \neq x^{*}$, and $u_{i}\left(\left(\sigma_{S}^{\prime}, \sigma_{-S}^{S, x^{*}}\right), x^{*}\right)>u_{i}\left(\left(\sigma_{S}, \sigma_{-S}^{S, x^{*}}\right), x^{*}\right)$ for all $i \in S$. Let $y=\sigma_{S}^{\prime}\left(x^{*}\right) \neq \sigma_{S}\left(x^{*}\right)$ and note that $S \in E\left(x^{*}, y\right)$. Suppose first that $S=S^{l}\left(x^{*}\right)$ for some $l=1, \ldots, k\left(x^{*}\right)-1$. Then $f^{l}\left(x^{*}\right)=\sigma_{S}\left(x^{*}\right) \neq y$, so by $\mathbf{M}$ there is $i \in S$ with

$$
\begin{aligned}
u_{i}\left(\left(\sigma_{S}, \sigma_{-S}^{S, x^{*}}\right), x^{*}\right) & =u_{i}\left(f^{l}\left(x^{*}\right), F\right) \geq u_{i}(y, F) \\
& =u_{i}\left(\left(\sigma_{S}^{\prime}, \sigma_{-S}^{S, x^{*}}\right), x^{*}\right)>u_{i}\left(\left(\sigma_{S}, \sigma_{-S}^{S, x^{*}}\right), x^{*}\right),
\end{aligned}
$$

which is impossible. So, suppose that $S \neq S^{l}\left(x^{*}\right)$ for all $l=1, \ldots, k\left(x^{*}\right)$. (Recall that $S \neq \emptyset=S^{k\left(x^{*}\right)}\left(x^{*}\right)$ by definition.) By the definition of $\succeq^{x^{*}}$ there are $l \leq k\left(x^{*}\right)$ and $i \in S$ such that $u_{i}\left(\left(\sigma_{S}^{0, x^{*}}, \sigma_{-S}^{S, x^{*}}\right), x^{*}\right)=u_{i}\left(f^{l}\left(x^{*}\right), F\right) \geq u_{i}(y, F)$. Hence,

$u_{i}\left(\left(\sigma_{S}^{0, x^{*}}, \sigma_{-S}^{S, x^{*}}\right), x^{*}\right) \geq u_{i}(y, F)=u_{i}\left(\left(\sigma_{S}^{\prime}, \sigma_{-S}^{S, x^{*}}\right), x^{*}\right)>u_{i}\left(\left(\sigma_{S}^{0, x^{*}}, \sigma_{-S}^{S, x^{*}}\right), x^{*}\right)$,

where the last inequality holds as $\sigma_{S}^{\prime}$ is unobjected against $\sigma_{-S}^{S, x^{*}}$ at $x^{*}$. But this is impossible. Thus, $\sigma$ is an equilibrium.

Example 5.15. Recall Example 5.6 and Figure 5. Depending on the order of moves in $v$ and $y$ we derive different equilibria and, hence, different rational extended expectation 
functions. Table 1 contains the four possible order profiles and the corresponding ESS's.

\begin{tabular}{cc}
$\succeq^{s}$ for $s \in\{v, y\}$ & ESS \\
\hline \hline$\{1\} \succeq^{v}\{2\},\{1\} \succeq^{y}\{2\}$ & $\{t, z\}$ \\
$\{1\} \succeq^{v}\{2\},\{2\} \succeq^{y}\{1\}$ & $\{t, z\}$ \\
$\{2\} \succeq^{v}\{1\},\{1\} \succeq^{y}\{2\}$ & $\{t, w, x, z\}$ \\
$\{2\} \succeq^{v}\{1\},\{2\} \succeq^{y}\{1\}$ & $\{t, z\}$ \\
\hline
\end{tabular}

Table 1: Order dependent equilibrium stable sets.

\subsection{APPLICATIONS}

\subsubsection{Absorbing extended expectation functions}

Players' utilities from an extended expectation function with non-terminal paths are lower than in any other state of the game. So, it is never in a coalition's interest to close a cycle or (if $X$ is infinite) play a strategy that leads to an infinite path. We can use this observation to prove the following corollary.

Corollary 5.1. Every rational extended expectation function is absorbing.

Proof. Let $F$ be rational. Then there is an equilibrium $\sigma$ with respect to some order profile $\succeq$ such that $F=F_{\sigma}$ by Theorem 5.1. Assume that there is $x \in X$ such that $P_{F}(x)$ is non-terminal. Define strategy profiles $\sigma^{1}, \ldots, \sigma^{k(x)-1}$ by

$$
\sigma_{T}^{l}(y)= \begin{cases}y & \text { if } y=x \text { and } T=S^{l^{\prime}}(x) \text { for some } l^{\prime}=1, \ldots, l \\ \sigma_{T}(y) & \text { otherwise. }\end{cases}
$$

That is, the only difference between $\sigma$ and $\sigma^{l}$ is that at $x$ the first $l$ coalitions that would move out of $x$ according to $\sigma$ do not do so according to $\sigma^{l}$. Let $l$ be the minimal integer such that the path $P_{F_{\sigma}}(x)$ is terminal. Such $l$ exists as $P_{F_{\sigma^{k}(x)-1}}(x)=(x)$. Let $S=S^{l}(x)$ and note that $\sigma_{-S}^{l}=\sigma_{-S}^{S, x}$ and $\sigma_{S}^{l}=\sigma_{S}^{0, x}$. By construction

$u_{i}\left(\left(\sigma_{S}^{0, x}, \sigma_{-S}^{S, x}\right), x\right)=u_{i}\left(\sigma^{l}, x\right)=U_{i}\left(t\left(P_{F_{\sigma^{l}}}(x)\right)\right)>-\infty=u_{i}\left(\left(\sigma_{S}, \sigma_{-S}^{S, x}\right), x\right)$.

But this means that $\sigma_{S}$ is objected given $\sigma_{-S}$, in contradiction to $\sigma$ 's being an equilibrium. 


\subsubsection{Single-Payoff Equilibrium Stable Sets}

A set $Y \subseteq X$ is a single-payoff set if $U_{i}(y)=U_{i}\left(y^{\prime}\right)$ for all $y, y^{\prime} \in Y$ and all $i \in N$. Dutta \& Vohra (2017) provide a detailed analysis of single-payoff SREFS's. We show that they actually coincide with single-payoff ESS's.

Theorem 5.2. Let $Y \subseteq X$ be a single-payoff set. Then $Y$ is an ESS if and only if $Y$ is a SREFS.

Proof. Let $Y$ be a single-payoff ESS, and let $F$ be a rational extended expectation function with $\mathcal{S}(F)=Y$. As each player's payoffs are identical across all elements of $Y$, and as $F$ satisfies $\mathbf{E}$, at each $x \in X$ there is at most one non-empty coalition that deviates from $x$ to some $z \in X \backslash\{x\}$, (All other moves would result in the same utility vector and, hence, be objected.) Hence, $k(x) \leq 2$ for all $x \in X$. By Corollary 5.1 the extended expectation function $F$ is absorbing, so by Lemma 5.1 the (basic) expectation function $F^{1}$ satisfies I', E', and M'. As the stationary points of $F$ and $F^{1}$ are identical, $Y$ is a REFS. By Theorem 1 in Dutta \& Vohra (2017), $Y$ is a SREFS.

Suppose now that $Y$ is a SREFS. Let $F^{\prime}$ be an absorbing rational (basic) expectation function with $Y$ as the set of stationary points, and let $F$ be the extended expectation function with $F^{1}(x)=F^{\prime}(x)$ for all $x \in X$ and $F^{2}(x)=(x, \emptyset)$ for all $x \in X \backslash Y$. Clearly, $\mathcal{S}(F)=Y$. As $k(x) \leq 2$ for all $x \in X$, conditions $\mathbf{M}$ and $\mathbf{E}$ of $F$ are equivalent to $\mathbf{M}$ ' and $\mathbf{E}^{\prime}$ of $F^{\prime}$. So, it remains to show that $F$ satisfies $\mathbf{I}$. Assume this were not true. Then there are $x, x^{\prime} \in X$ and $T \in E\left(x, x^{\prime}\right)$ such that $u_{i}\left(x^{\prime}, F\right)>u_{i}\left(f^{l}(x), F\right)$ for $l=1,2$ and all $i \in T$. Note that $u\left(f^{l}(x), F\right)=u(x, F)$ as either $l=k(x)$ and, hence, $f^{l}(x)=x$ and $u(x, F)=U(x)$, or $k(x)=2$ and $l=1$ in which case $t\left(P_{F}(x)\right)=$ $t\left(P_{F}\left(f^{l}(x)\right)\right)$ by definition. Let $y=t\left(P_{F}\left(f^{l}(x)\right)\right)$ and $y^{\prime}=t\left(P_{F}\left(x^{\prime}\right)\right)$ and note that $y, y^{\prime} \in Y$. ( $y$ and $y^{\prime}$ exist as $F^{\prime}$ is absorbing and $k(x) \leq 2$ for all $x \in X$.) Then $U_{i}\left(y^{\prime}\right)=u_{i}\left(x^{\prime}, F\right)>u_{i}\left(f^{l}(x), F\right)=u_{i}(x, F)=U_{i}(y)=U_{i}\left(y^{\prime}\right)$. This, however, is impossible. So, $F$ satisfies $\mathbf{I}$.

\subsubsection{Characteristic function form games}

A game in characteristic function form (with player set $N$ ) is a map $V$ that assigns to each nonempty $S \subseteq N$ a comprehensive, closed, and convex set $V(S) \subseteq \mathbb{R}^{S}$. Ray \& Vohra (2015) associate with such a game $V$ an abstract game $\left(N, X, E,\left(U_{i}(.)\right)_{i \in N}\right)$ as follows: a state $x \in X$ is a pair $(v(x), \pi(x))$ of a partition $\pi(x)$ of the player set and a utility vector $v(x)$ with $v_{S}(x) \in V(S)$ for all $S \in \pi(x)$. The utility function $U_{i}$ is simply defined as $U_{i}(x)=v_{i}(x)$. The effectivity correspondence $E$ satisfies the following two conditions: 
(i) If $T \in E(x, y), S \in \pi(x)$, and $S \cap T=\emptyset$ then $S \in \pi(y)$ and $v_{S}(y)=v_{S}(x)$.

(ii) For each $x \in X$, each $T \subseteq N$, and each weakly Pareto efficient $v \in V(T)$ there is $y \in X$ such that $T \in E(x, y), T \in \pi(y)$, and $v_{T}(y)=v$.

Condition (i) ensures that a deviating coalition $T$ cannot affect the partition or the payoffs in coalitions it does not intersect with at state $x$. Condition (ii) ensures that coalition $T$ can, from any state $x$, deviate to a state with $T$ as a member of the partition and $v$ as payoff vector for $T$, as long as $v$ is feasible and weakly Pareto efficient for $T$.

Corollary 5.2. Let $V$ be a game in characteristic function form and let $G$ be an abstract game that satisfies Conditions ( $i$ ) and (ii). If $Y$ is a single-payoff ESS with payoff vector $v \in \mathbb{R}^{N}$ then $v$ lies in the coalition structure core of $V$.

Proof. By Theorem 5.2 any single-payoff ESS is a single-payoff SREFS. By Theorem 2 in Ray \& Vohra (2015) this is the case if and only if $v$ is separable, so $v$ must lie in the coalition structure core of $V$.

\subsubsection{Matching}

We consider two-sided one-to-one matching problems. Let $W$ be a set of women, $M$ be a set of men, and $N=W \cup M$. For each $i \in W$ let $\succeq_{i}^{\prime}$ be a strict preference order over $M \cup\{i\}$, and for $j \in M$ let $\succeq_{j}^{\prime}$ be a strict preference order over $M \cup\{j\}$. A matching is a map $\mu: N \rightarrow N$ such that $\mu(i) \in M \cup\{i\}$ for all $i \in W, \mu(j) \in W \cup\{j\}$ for all $j \in M$, and $\mu(\mu(i))=i$ for all $i \in N$. If $\mu(i)=i$ then $i$ is single, otherwise $i$ is matched to $\mu(i)$. A matching $\mu$ is individually rational if $\mu(i) \succeq_{i}^{\prime} i$ for all $i \in N$. A pair $(i, j) \in W \times M$ blocks a matching $\mu$ if $j \succ_{i}^{\prime} \mu(i)$ and $i \succ_{j}^{\prime} \mu(j)$. A matching $\mu$ is stable if it is individually rational and not blocked by any pair.

Let $\mathcal{M}$ be the set of all matchings, and define each player $i$ 's preference $\succeq_{i}$ over $\mathcal{M}$ by $\mu \succeq_{i} \mu^{\prime}$ if and only if $\mu(i) \succeq_{i}^{\prime} \mu^{\prime}(i)$. Define an effectivity correspondence $E: \mathcal{M} \times \mathcal{M} \rightarrow$ $2^{N}$ by

$$
E\left(\mu, \mu^{\prime}\right)=\left\{S \subseteq N: \begin{array}{l}
\left\{i, \mu^{\prime}(i)\right\} \subseteq S \text { whenever } \mu^{\prime}(i) \notin\{i, \mu(i)\}, \text { and } \\
\{i, \mu(i)\} \cap S \neq \emptyset \text { whenever } \mu^{\prime}(i)=i \neq \mu(i)
\end{array}\right\}
$$

That is, $E\left(\mu, \mu^{\prime}\right)$ contains all those coalitions $S$ whose members can transform $\mu$ into $\mu^{\prime}$ via deleting old and forming new links. The tupel $\left(N, \mathcal{M}, E,\left(\succeq_{i}\right)_{i \in N}\right)$ forms an abstract game. ${ }^{8}$

Corollary 5.3. Let $\mu \in \mathcal{M}$. Then $\mu$ is a stable matching if and only if $\{\mu\}$ is an ESS of the abstract game.

\footnotetext{
${ }^{8} \mathrm{As} \mathcal{M}$ is finite $\succeq_{i}$ is equivalent to an ordinal utility function.
} 
Proof. Theorems 1 and 2 in Mauleon et al. (2011) show that $\mu$ is stable if and only if $\{\mu\}$ is a farsighted stable set. Theorem 1 of Dutta \& Vohra (2017) shows that any singlepayoff farsighted stable set is a SREFS, and any single-payoff SREFS is a farsighted set. Hence, our Theorem 5.2 completes the proof.

\subsection{CONCLUSION}

Extended expectation functions provide a tool to find stable outcomes in games with fully farsighted players, that is, players who do not only take into account what happens after they move from one state to another, but also what happens if they do not move. Moreover, they have a clear non-cooperative foundation, and the three rationality axioms, Internal Stability, External Stability, and Maximality, can be interpreted in terms of equilibrium behavior of coalitions. Hence, this chapter closes the gap in farsightedness that Chwe has formulated more than two decades ago (cf. Chwe, 1994); and, at the same time, provides a bridge between cooperative and non-cooperative perspectives on abstract games. We thereby reconcile central concepts of both worlds, namely dominance and rational strategies.

FURTHER RESEARCH From here many avenues for further research present themselves, and we shall outline some of them. First, rational extended expectation functions do not always exist; the classical roommate problem is an easy example for non-existence.

Example 5.16. Consider the roommate problem in Figure 11 with $0<a<4$. For the corresponding abstract game there is no rational extended expectation function: Clearly, $F$ with $F^{1}(x)=(x, \emptyset)$ for all $x \in X$ is not rational. Neither is it rational to have a cycle. So, suppose without loss of generality that $x$ is stationary. Then $\{1,2\}$ has to move from $z$ to $x$ by $\boldsymbol{I}$. Hence, $y$ must be stationary by $\boldsymbol{E}$ as 3 would object the move to $z$. But if $y$ is stationary, $\{2,3\}$ must move from $x$ to y by $\boldsymbol{I}$, contradicting $x$ 's being stationary.

If one assumes that in a cycle players' payoffs are not $-\infty$ but rather an expected value of payoffs that are achievable, one can obtain a rational, non-absorbing, extended expectation function for $a \leq 2$ in which all coalitions move in all states and the expected payoff is $\frac{4+a}{3}$ for all players. In this case there is no stationary point; instead one is left with a stationary distribution over outcomes. But for $a>2$ even this fails for the same reason as in Example 5.16 as $a>\frac{4+a}{3}$. Bearing in mind that there is always a non-cooperative equilibrium behind a rational extended expectation function, one might wonder whether mixed strategies could help? And indeed: if each coalition moves with probability $p=\frac{4}{a}-1$ (recall $a>2$ ) then player 2 is exactly indifferent between moving from $x$ to $y$ and staying in $x$, and the same is true for player 1 in $z$ and player 3 in $y$. Whether such a mixed strategy equilibrium always exists is, however, unclear. 
Figure 11: The Roommate Problem

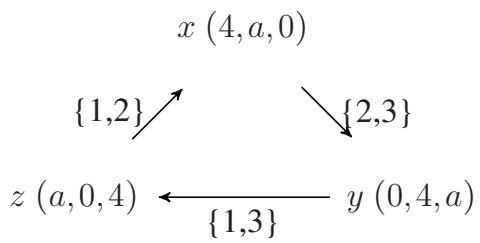

The second problem that has to be solved is a clear description of the ESS in various settings. While we have covered single payoff ESS's to some extend, we do not know yet the general structure for arbitrary games in characteristic function form.

Third, we feel that the idea of expectation functions, both basic and extended, heavily relies on players' and coalitions' knowledge about how the game is being played. We therefore think that next to the axiomatic and the non-cooperative equilibrium foundation, one should aim for an epistemic foundation as well.

Finally, while we impose an exogenous order profile $\succeq$, one might assume that the order in state $x$ depends on how $x$ has been implemented. In the context of abstract games this has recently been investigated by Dutta \& Vartiainen (2017) and Vartiainen (2018). In a framework without coalitional moves, this question has been targeted by Brams (1994) and, more recently, by Brams \& Ismail (2018). 


\subsection{INTRODUCTION}

In many daily-life situations people have to cooperate with each other, and often, repeatedly over time. Such situations can be modeled with tools of cooperative game theory. However, finding a unified approach for solving such dynamic cooperative games seems challenging. Issues such as variation in the population or changes in the characteristic function-due to endogenous or exogenous influences - are just a few issues that might arise in a dynamic setting. Some literature has been facing these matters. For instance, Rosenthal (1990a) and Rosenthal (1990b) allow for variations in the population but assume the characteristic function to be constant over time. Other papers, such as Lehrer \& Scarsini (2013) and Filar \& Petrosjan (2000) allow for variations in the characteristic function. On one hand, Lehrer \& Scarsini (2013) consider dynamic cooperative games, where the worth of coalitions varies over time based on the history of allocations, in particular, they consider Markovian dynamic cooperative games. On the other hand, Filar $\&$ Petrosjan (2000) consider dynamic cooperative games where each stage game may depend on the payoffs obtained in earlier periods. They model the endogenous changes of the characteristic functions via differential and difference equations. While Lehrer \& Scarsini (2013) are interested in defining a new core concept, the intertemporal core, Filar \& Petrosjan (2000) focus on analyzing time consistency of solution concepts for the stage games. Predtetchinski (2007) deals with infinite-horizon stationary cooperative games, where at each moment the game is in one of a finite number of states that determines which instantaneous game is played. The states evolve according to an exogenous Markov chain and they do not depend on past allocations. In Kranich et al. (2005) the sequence of transferable utility games (TU-games) is fixed and exogenously given, and so is the set of players; these assumptions are also made in Habis \& Herings (2010) who provide a different definition of the weak sequential core introduced in Kranich et al. (2005). We follow this approach. Normally, a solution concept for a dynamic cooperative game specifies a payoff stream, and independent of how the sequence of games arises, players have to be endowed with intertemporal utilitiy functions in order to evaluate these payoffs. Most of the literature on dynamic cooperative games focuses on introducing and elaborating on different core concepts. We take a different approach in this chapter. We apply a point-wise solution to each stage game and assume that agents can make 
transfers among periods and hence affect their payoffs. The chapter starts from Berden (2007) and extends her work in several aspects.

In cooperative game theory it is assumed that agents make binding contracts, and, in particular, it abstracts away from any strategic interaction between players. However, when cooperating repeatedly over time, agents might try to maximize their own benefit (in terms of money, power, etc.) and would probably act strategically if they could. The classical assumptions of cooperative game theory do not take into account such strategic behavior. We intend to incorporate this strategic behavior by modeling a noncooperative game based on a sequence of transferable utility games and point-wise solution concepts for them. We assume that the sequence of TU-games is finite and exogenously specified, the player set is fixed and a solution concept is applied to each stage game separately. Moreover, we endow agents with intertemporal utility functions to enable them to evaluate the generated payoff streams. We then allow agents to redistribute some worth (that will be specified by the TU-game and the particular transfer system) by making transfers among periods. As a starting point, we model three different transfer systems. Under the first transfer system, agents can only influence their individual worth by making transfers among periods and the worth of non-singleton coalitions remain untouched. Consider the example of a group of researchers working together on several papers over time. Suppose that each of them could write papers on their own, but if they cooperate they produce papers of higher quality. Further suppose that the first researcher decides to focus less on the first and more on the second paper, but only if she had to work alone. This affects the characteristic functions of the dynamic game so that only her individual worth in the stage games changes. This idea is modeled through the first system. However, suppose aforementioned researcher cannot work as intensively as she wanted on the first paper due to time constraints, or because she is occupied with other work. Then one could argue that her contribution to the first paper will diminish, not only if she worked alone, but also if she worked with others. Transformed into the language of cooperative game theory, this means that not only her individual worth will change, but also the worth of coalitions that contain her. We model this idea in two different ways. In the second system an individual's transfer also influences the worth of all coalitions which contain her. We assume that the change of a coalition's worth equals the sum of the changes of the individual worths of players contained in this coalition. The third transfer system allows agents to affect coalitions' worth to the extent of their marginal contribution to them.

Obviously, by conducting transfers according to the above transfer systems players indirectly affect their payoffs. We model non-cooperative games which depend on the dynamic TU-game, the solution applied to each game, and the transfer system considered. Our main goal is to identify Nash equilibria in these non-cooperative games. Typically, in a non-cooperative game players simultaneously choose their strategies. 
Hence, the payoff of the players depends on the strategies chosen by themselves and by the other players. It is assumed that feasible strategies of a player are independent of the choice of strategies of the other players. As Morales \& Vermeulen (2009) remark, in a variety of real life problems the set of feasible strategies of an agent depends on the strategies played by the others. ${ }^{1}$ As it turns out in some of the non-cooperative games introduced in this chapter (again, this depends on the transfer system considered) there are constraints across strategies of the players, i.e. which of a player's strategies are feasible depends on the strategies chosen by the other players. If there are feasibility constraints across players' strategies existence of Nash equilibria cannot be shown by applying Kakutani's fixed point theorem. However, Morales \& Vermeulen (2009) argue that in such games players avoid infeasibility in equilibrium. ${ }^{2}$ They introduce games played in a constrained environment and prove a generalization of the theorem of Nash (Nash, 1950b, 1951). We follow their approach by transforming some of the above non-cooperative games into games played in a constraint environment, and then prove existence of Nash equilibrium in these games by applying Theorem 1 of Morales \& Vermeulen (2009). We identify transfer-concavity of the solution for the different transfer systems as a sufficient condition for existence of a Nash equilibrium when the utility function of each agent is increasing and quasiconcave (Theorem 6.2). A solution satisfies transfer-concavity with respect to a transfer system, if it is concave on the class of all games that can be generated by transfers according to this particular system. We further show that the $\tau$-value (cf. Tijs, 1981) is not transfer-concave under the first transfer system. Moreover, we show that a Nash-equilibrium need not exist under the first transfer system if the nucleolus (cf. Schmeidler, 1969) is applied to each stage game. This is consistent with the observation that the nucleolus is not concave under the first transfer system. ${ }^{3}$ We provide characterizations of the Nash equilibria for all games considered and also discuss uniqueness of Nash equilibria. We then show that there are classes of TU-games where other transfer systems seem in order. As an example we discuss market and voting games. Again, we model non-cooperative games, allowing agents to make transfers among periods. In the first case, agents can redistribute their initial endowments among periods and in the second, agents can relocate their voting weights. While for the game based on market games we prove existence, we show that in the case of voting games Nash equilibria need not exist. Also uniqueness of the equilibria is discussed.

We want to point out that although we interpret the sequence of TU-games in terms of games played in different periods our model is not limited to this interpretation. It also

\footnotetext{
${ }^{1}$ We refer the reader to Morales \& Vermeulen (2009) for explicit examples.

${ }^{2}$ This result is independent of the way how infeasibility is treated in the game, as long as it is treated correctly, e.g. use monetary punishment for infeasible choices.

${ }^{3} \mathrm{~A}$ result provided in Berden (2007).
} 
encompasses situations where the same group of agents cooperates on different projects at the same time.

The chapter is structured as follows. Section 6.2 provides necessary notation and presents the main ingredients of our model. In Section 6.3 we introduce strategic interaction between players to dynamic TU-games. We present three different transfer systems and prove existence of Nash equilibria and characterizations of the corresponding equilibria. Section 6.4 focuses on market games and transfers of initial endowments, while Section 6.5 discusses dynamic voting games. We conclude and discuss avenues for further research in Section 6.6.

\subsection{PRELIMINARIES}

In a first step we provide some necessary notation discussing TU-games and solution concepts, intertemporal utility functions and games played in a constrained environment.

\subsubsection{Transferable utility games}

A game with transferable utility consists of a pair $(N, v)$ where $N:=\{1, \ldots, n\}$ is the finite set of players and $v: 2^{N} \rightarrow \mathbb{R}$ assigns to each coalition $S \subseteq N$ the worth $v(S)$ with $v(\emptyset)=0$. For simplicity we write $v(i)$ instead of $v(\{i\})$ for all $i \in N$. Further, we omit $N$ from notation and simply write $v$. The set of all TU-games with player set $N$ is denoted by $\mathcal{G}^{N}$. The zero-game is denoted by $z \in \mathcal{G}^{N}$, i.e. $z(S)=0$ for all $S \subseteq N$. Let the games $1_{T}, 2_{T} \in \mathcal{G}^{N}$ for $\emptyset \neq T \subseteq N$ be defined as follows. The game $1_{T}$ has worth $1_{T}(S)=1$ if $S=T$ and $1_{T}(S)=0$ otherwise. The game $2_{T}$ has worth $2_{T}(S)=1$ if $T \subseteq S$ and $2_{T}(S)=0$ otherwise. Note that the game $2_{T}$ is the usual unanimity game on $T$. For simplicity, whenever $T=\{i\}$ for $i \in N$, we write $1_{i}$ and $2_{i}$, respectively.

A game $v \in \mathcal{G}^{N}$ is additive if $v(S \cup T)=v(S)+v(T)$ for all $S, T \subseteq N$ such that $S \cap$ $T=\emptyset$. Hence, the additive game $v \in \mathcal{G}^{N}$ is generated by the vector $(v(1), \ldots, v(n)) \in$ $\mathbb{R}^{N}$. Let $\mathcal{B} \subseteq 2^{N} \backslash\{\emptyset\}$ be a collection of nonempty coalitions in the player set $N$. The collection $\mathcal{B}$ is balanced if there exist numbers $\lambda_{S}>0$ for $S \in \mathcal{B}$ such that for every player $i \in N$ :

$$
\sum_{S \in \mathcal{B}: i \in S} \lambda_{S}=1
$$

A game $v$ is balanced if

$$
\sum_{S \in \mathcal{B}} \lambda_{S} v(S) \leq v(N)
$$

holds for every balanced set $\mathcal{B}$ with weights $\left\{\lambda_{S}\right\}_{S \in \mathcal{B}}$. 
A solution concept assigns to each $v \in \mathcal{G}^{N}$ a vector or a set of vectors in $\mathbb{R}^{N}$. One of the most prominent solution concepts for TU-games is the core, introduced in Gillies (1959). The core is defined as follows:

$$
\begin{gathered}
C(v):=\left\{\mathbf{x} \in \mathbb{R}^{\mathbb{N}} \mid \sum_{i \in S} x_{i} \geq v(S) \text { for all } S \subseteq N\right. \text { and } \\
\left.\sum_{i \in N} x_{i}=v(N)\right\} .
\end{gathered}
$$

Suppose $\mathbf{x} \in C(v)$. If $\mathbf{x}$ is the proposed payoff vector, then no coalition $S \subseteq N$ has an incentive to split off, as the total amount $x(S):=\sum_{i \in S} x_{i}$ allocated to $S$ is not smaller than the amount $v(S)$ which they can obtain by forming a subcoalition.

A one-point solution concept, i.e. a solution concept that assigns a unique payoff distribution to a TU-game, is also called a value. A value $\Phi: \mathcal{G}^{N} \rightarrow \mathbb{R}^{N}$ assigns to a TU-game $v \in \mathcal{G}^{N}$ the vector $\Phi(v)$. We say $\Phi$ is additive if for all $v, w \in \mathcal{G}^{N}$ we have that $\Phi(v+w)=\Phi(v)+\Phi(w)$. A value $\Phi$ is [strictly] increasing in the worth of coalition $T \subseteq N$ if the following condition is satisfied: If $v, v^{\prime} \in \mathcal{G}^{N}$ such that $v(T)>v^{\prime}(T)$ and $v(S)=v^{\prime}(S)$ for all $S \neq T$, then $\Phi_{i}(v) \geq \Phi_{i}\left(v^{\prime}\right)\left[\Phi_{i}(v)>\Phi_{i}\left(v^{\prime}\right)\right]$ for all $i \in T$. We say $\Phi$ is [strictly] decreasing in the worth of coalition $T$ if $-\Phi$ is [strictly] increasing in the worth of coalition $T . \Phi$ is concave if $\Phi(\lambda v+(1-\lambda) w) \geq \lambda \Phi(v)+(1-\lambda) \Phi(w)$ for all $v, w \in \mathcal{G}^{N}$ and $\lambda \in(0,1)$. Let $v, w \in \mathcal{G}^{N}$ and $a \in \mathbb{R}$. $\Phi$ is linear if $\Phi(v+w)=$ $\Phi(v)+\Phi(w)$ and $\Phi(a v)=a \Phi(v)$. Let $\bar{a}, v \in \mathcal{G}^{N}$ with $\bar{a}$ being the additive game generated by the vector $\mathbf{a}=\left(a_{1}, \ldots, a_{n}\right) \in \mathbb{R}^{N}$, we say $\Phi$ is relatively invariant under strategic equivalence if $\Phi(\lambda v+\bar{a})=\lambda \Phi(v)+\mathbf{a}$ for every $\lambda \in(0, \infty){ }^{4}$

We now give definitions of values for TU-games.

\section{Shapley value}

The Shapley value, introduced in Shapley (1953a), is defined as

$$
S h_{i}(v):=\frac{1}{n !} \cdot \sum_{S \subseteq N \backslash\{i\}}(n-|S|-1) ! \cdot|S| ! \cdot(v(S \cup\{i\})-v(S)) .
$$

Hence, the Shapley value assigns to each player his average marginal contribution in the game. The Shapley value is defined for any class of TU-games. An axiomatic characterization of the Shapley value is given in Shapley (1953b).

\section{Egalitarian Shapley values}

The class of egalitarian Shapley values, introduced in Joosten (1996) also satisfies

\footnotetext{
${ }^{4}$ This condition is also known under the name covariance. See for example Schmeidler (1969) and Sobolev (1975).
} 
many desirable properties. ${ }^{5}$ The egalitarian Shapley values represent the trade-off between marginalism and egalitarianism. The equal division solution distributes the worth $v(N)$ equally among all players, i.e.,

$$
E D_{i}(v):=\frac{v(N)}{|N|} \text { for all } i \in N .
$$

The egalitarian Shapley values are defined as convex combinations of the Shapley value and the equal division solution. Hence, for every $\lambda \in[0,1]$, the egalitarian Shapley value $S h^{\lambda}$ is given by

$$
S h^{\lambda}(v)=\lambda S h(v)+(1-\lambda) E D(v)
$$

Axiomatic characterizations are given for example in Joosten (1996), van den Brink et al. (2013) and Casajus \& Hüttner (2013).

\section{Consensus value}

The consensus value was introduced and axiomatically characterized in Ju et al. (2007). It is based on a recursive two-sided negotiation process to establish cooperation between all players. Let $\Sigma(N)$ be the set of all bijections $\sigma: N \rightarrow N$. For a given $\sigma \in \Sigma(N)$ and $k \in N$ let $S_{k}^{\sigma}:=\{\sigma(1), \ldots, \sigma(k)\} \subset N$ and $S_{0}^{\sigma}:=\emptyset$. Let $v \in \mathcal{G}^{N}$. Recursively,

$$
r\left(S_{k}^{\sigma}\right):= \begin{cases}v(N) & \text { if } k=|N| \\ v\left(S_{k}^{\sigma}\right)+\frac{1}{2}\left(r\left(S_{k+1}^{\sigma}\right)-v\left(S_{k}^{\sigma}\right)-v(\{\sigma(k+1)\})\right. & \text { otherwise }\end{cases}
$$

where $r\left(S_{k}^{\sigma}\right)$ is the standardized remainder for coalition $S_{k}^{\sigma}$. The individual standardized remainder vector $s^{\sigma}(v)$ is defined as follows

$$
s_{\sigma(k)}^{\sigma}:= \begin{cases}v(\{\sigma(k)\})+\frac{1}{2}\left(r\left(S_{k}^{\sigma}\right)-v\left(S_{k-1}^{\sigma}-v(\{\sigma(k)\})\right)\right. & \text { if } k \in\{2, \ldots,|N|\} \\ r\left(S_{1}^{\sigma}\right) & \text { otherwise }\end{cases}
$$

For every $v \in \mathcal{G}^{N}$, the consensus value $\Psi(v)$ is defined as the average over $\Sigma(N)$ of all bijections of the individual standardized remainder vectors, i.e.

$$
\Psi(v):=\frac{1}{|N| !} \sum_{\sigma \in \Sigma(N)} s^{\sigma}(v)
$$

\footnotetext{
${ }^{5}$ Although, originally introduced to the literature as $\alpha$-egalitarian Shapley values, we refer to them simply as egalitarian Shapley values in order to avoid confusion in the sequel of the chapter.
} 
Hence, the consensus value can be interpreted as the expected individual standardized remainder a player can get by participating in coalitions.

\section{Nucleolus and prenucleolus}

The nucleolus and prenucleolus are both defined as the solution of a minimization problem, where excesses of all non-empty coalitions of a game are lexicographically minimized. The excess of a non-empty coalition $S$ in game $v \in \mathcal{G}^{N}$ given a payoff vector $\mathbf{x}=\left(x_{1}, \ldots, x_{n}\right) \in \mathbb{R}^{N}$ is defined as

$$
e(S, \mathbf{x}):=v(S)-\sum_{i \in S} x_{i}
$$

for all $v \in \mathcal{G}^{N}$. The excess is the difference between what $S$ can acquire on its own and what it receives in total from $\mathrm{x}$. One interpretation of the excess is that it reflects the dissatisfaction of $S$ given $\mathbf{x}$. Let $\theta(\mathbf{x})$ be a vector containing the excesses of all coalitions arranged in non-increasing order,

$$
\theta(\mathbf{x}):=\left(e\left(S_{1}, \mathbf{x}\right), \ldots, e\left(S_{2^{|N|}-1}, \mathbf{x}\right)\right)
$$

We say $\theta(\mathbf{y})$ is lexicograhically larger than $\theta(\mathbf{x})$, denoted by $\theta(\mathbf{y}) \succeq^{\text {lex }} \theta(\mathbf{x})$, if a positive integer $q \leq 2^{|N|}-1$ exists, such that $\theta_{i}(\mathbf{x})=\theta_{i}(\mathbf{y})$ whenever $i<q$ and $\theta_{q}(\mathbf{y})>\theta_{q}(\mathbf{x})$. Thus the nucleolus is defined as

$$
\nu(v):=\left\{\mathbf{x} \in I(v) \mid \theta(\mathbf{y}) \succeq^{l e x} \theta(\mathbf{x}) \text { for all } \mathbf{y} \in I(v)\right\}
$$

with $I(v)=\left\{\mathbf{x} \in \mathbb{R}^{N} \mid x_{i} \geq v(i)\right.$ for all $i \in N$ and $\left.\sum_{i \in N} x_{i}=v(N)\right\}$ being the set of imputations. Thus, the motivation behind the nucleolus is that it minimizes the dissatisfaction of the coalition treated the worst. Observe that the nucleolus of a game $v \in \mathcal{G}^{N}$ is defined only if $I(v) \neq \emptyset$. The prenucleolus is defined as

$$
\nu^{*}(v):=\left\{\mathbf{x} \in I^{*}(v) \mid \theta(\mathbf{y}) \succeq^{l e x} \theta(\mathbf{x}) \text { for all } \mathbf{y} \in I^{*}(v)\right\}
$$

with $I^{*}(v)=\left\{\mathbf{x} \in \mathbb{R}^{\mathbb{N}} \mid \sum_{i \in N} x_{i}=v(N)\right\}$ being the set of efficient payoff distributions. We refer the reader to Schmeidler (1969) and Sobolev (1975) for axiomatic characterizations.

\section{$\tau$-value}

The $\tau$-value was introduced in Tijs (1981) and axiomatically characterized in Tijs (1987). For $v \in \mathcal{G}^{N}$ we denote by $M(v)$ the marginal vector, with the $i$ th coordinate

$$
M_{i}(v)=v(N)-v(N \backslash\{i\})
$$




\begin{tabular}{lcc} 
Solution concept & linearity & relative invariance under s-equivalence \\
\hline \hline Shapley value & yes & yes \\
Egalitarian Shapley values & yes & no \\
Consensus value & yes & yes \\
Nucleolus & no & yes \\
Prenucleolus & no & yes \\
$\tau$-value & no & yes \\
\hline
\end{tabular}

Table 2: Linearity and relative invariance under strategic equivalence

Moreover let $R_{v}(S, i)=v(S)-\sum_{j \in S \backslash\{i\}} M_{j}(v)$ denote the remainder of agent $i$ in coalition $S$ at game $v \in \mathcal{G}^{N}$. We call

$$
m_{i}(v)=\max _{S \ni i} R_{v}(S, i)
$$

the minimal right of agent $i$ and denote the minimal right vector by $m(v)=$ $\left(m_{1}(v), \ldots, m_{n}(v)\right)$. The $\tau$-value is defined as follows:

$$
\tau(v):=m(v)+\beta_{v}(M(v)-m(v))
$$

with $\beta_{v}=\left(\sum_{i=1}^{n} M_{i}(v)-\sum_{i=1}^{n} m_{i}(v)\right)^{-1}\left(v(N)-\sum_{i=1}^{n} m_{i}(v)\right)$ if $M(v) \neq m(v)$ and $\beta_{v}=0$ otherwise. The $\tau$-value is defined for quasi-balanced games. A game $v \in \mathcal{G}^{N}$ is called quasi-balanced if and only if

$$
m(v) \leq M(v) \text { and } \sum_{i \in N} m_{i}(v) \leq v(N) \leq \sum_{i \in N} M_{i}(v)
$$

As linearity and relative invariance under strategic equivalence will be of main interest in the sequel of the chapter, Table 2 illustrates which of the above values satisfies one or both properties.

\subsubsection{Intertemporal utility functions}

Let $u: \mathbb{R}^{T} \rightarrow \mathbb{R}$ be an intertemporal utility function. We say $u$ is [strictly] increasing if for all $\mathbf{x}, \mathbf{y} \in \mathbb{R}^{T}$ with $\mathbf{x}>\mathbf{y}$ it holds that $u(\mathbf{x}) \geq u(\mathbf{y})[u(\mathbf{x})>u(\mathbf{y})]{ }^{6}$ The function $u$ is quasi-concave if for all $\mathbf{x}, \mathbf{y} \in \mathbb{R}^{T}$ and $\lambda \in(0,1)$ we have that $u(\lambda \mathbf{x}+(1-\lambda) \mathbf{y}) \geq$ $\min \{u(\mathbf{x}), u(\mathbf{y})\}$. The function $u$ is concave if for all $\mathbf{x}, \mathbf{y} \in \mathbb{R}^{T}$ and $\lambda \in(0,1)$ we have that $u(\lambda \mathbf{x}+(1-\lambda) \mathbf{y}) \geq \lambda u(\mathbf{x})+(1-\lambda) u(\mathbf{y})$. Obviously, every concave utility

\footnotetext{
${ }^{6}$ We write $\mathbf{x} \gg \mathbf{y}$ if $x^{t}>y^{t}$ for all $t \in\{1, \ldots, T\} ; \mathbf{x}>\mathbf{y}$ if $x^{t} \geq y^{t}$ for all $t \in\{1, \ldots, T\}$ and $x^{t}>y^{t}$ for at least one $t \in\{1, \ldots, T\}$; and $\mathbf{x} \geq \mathbf{y}$ if $x^{t} \geq y^{t}$ for all $t \in\{1, \ldots, T\}$.
} 
function is quasiconcave.

\subsubsection{Games played in a constrained environment}

Each $i \in N$ has a finite set $A_{i}$ of one-dimensional decision variables at his disposal. A strategy of player $i$ is then a vector

$$
x_{i}=\left(x_{i a}\right)_{a \in A_{i}}
$$

in $\mathbb{R}^{A_{i}}$. Due to feasibility constraints not all strategy combinations $x=\left(x_{i}\right)_{i \in N}$ may be realized. Hence, let

$$
\mathcal{D} \subseteq \prod_{i \in N} \mathbb{R}^{A_{i}}
$$

be the collection of feasible strategy combinations. We assume that $\mathcal{D}$ is nonempty, compact and convex. We call a nonempty, compact and convex set $\mathcal{D}$ of feasible strategy combinations a constrained environment. Consider player $j$. Given a strategy combination $x=\left(x_{i}\right)_{i \in N}$ the set of feasible responses of player $j$ is

$$
\mathcal{D}_{j}(x):=\left\{y \in \mathcal{D} \mid y_{i}=x_{i} \text { for all } i \neq j\right\}
$$

Suppose a best response correspondence $B R_{i}: \mathcal{D} \rightrightarrows \mathcal{D}$ is given for player $i$ such that

1. the graph of $B R_{i}$ is a closed subset of $\mathcal{D} \times \mathcal{D}$, and

2. for each $x \in \mathcal{D}, B R_{i}(x)$ is a non-empty subset of $\mathcal{D}_{i}(x)$.

Observe that above definition of is slightly different from the usual terminology. In the classical normal-form setting best responses are a subset of the space of strategy profiles. However, because of the restrictions on the strategy space that is added here this is no longer the case. Therefore, the usual tools, like for example the fixed point theorem of Kakutani, no longer apply.

A player $i$ is said to behave optimally when $x \in B R_{i}(x)$.

Definition 6.1. A strategy profile $x \in \mathcal{D}$ is called a Nash equilibrium of the game in a constraint environment if for all players $i$ we have that $x \in B R_{i}(x)$.

Definition 6.2. The object $G=\left(\mathcal{D},\left(B R_{i}\right)_{i \in N}\right)$ is called a game played in a constrained environment.

Theorem 6.1 (Morales \& Vermeulen (2009)). Let G be a game played in a constrained environment. If for each $i \in N$ the best response correspondence is convex-valued, then the game has at least one Nash equilibrium. 


\subsection{INDIVIDUAL TRANSFERS AND NASH EQUILIBRIUM}

We consider a finite sequence of TU-games, $v^{1}, \ldots, v^{T}$ with $T \geq 1$. We assume that $v^{t}(S) \geq 0$ for all $S \subseteq N$ and all $t \in\{1, \ldots, T\}$. Although this assumption is not commonly made, we want to point out that it is reasonable in our model. The main interpretation that we have in mind is that individuals can redistribute some worth among periods. Specifically, through transfers we introduce the possibility of saving and borrowing to our model. In order to avoid the possibility that individuals can borrow an infinite amount in some period, we need a lower bound on the worth that a coalition can feasibly achieve. In this chapter, we use a normalization and set this lower bound to be zero. This leads, in particular, to the effect that players can only borrow from their own future. Hence, by making this assumption we abstract away from the possibility of borrowing from a third party and the possibility of going bankrupt. Each player $i \in N$ has an intertemporal utility function $u_{i}: \mathbb{R}^{T} \rightarrow \mathbb{R}$ that assigns to every payoff stream $\mathbf{x}_{i}=\left(x_{i}^{1}, \ldots, x_{i}^{T}\right) \in \mathbb{R}^{T}$ of player $i$ utility $u_{i}\left(\mathbf{x}_{i}\right)$.

Definition 6.3. A dynamic TU-game $\Gamma$ is a pair $(\mathbf{v}, \mathbf{u})$ where

1. $\mathbf{v}=\left(v^{1}, \ldots, v^{T}\right) \in\left(\mathcal{G}^{N}\right)^{T}$ and

2. $\mathbf{u}=\left(u_{1}, \ldots, u_{n}\right)$.

A solution $\Phi$ is applied to dynamic TU-games. This means that $\Phi$ is applied to each stage game within the dynamic TU-game separately, generating some payoff stream. We assume that players can make transfers among TU-games within the dynamic game, that are specified by different transfer systems. By doing so, individuals can affect their intertemporal payoffs. We discuss three different transfer systems and model non-cooperative games based on them.

\subsubsection{Strategic interaction}

In order to define non-cooperative games as described above, we have to introduce a little more notation. We will denote transfers of agent $i$ at period $t$ by $\alpha_{i}^{t}$ or $\alpha_{i, S}^{t}$ depending on the transfer system considered. Negative $\alpha_{i}^{t}\left(\alpha_{i, S}^{t}\right)$ represent borrowing from period $t$ positive $\alpha_{i}^{t},\left(\alpha_{i, S}^{t}\right)$ represent additional worth in period $t$. The resulting game after transfers $\alpha$ is denoted by $v_{\alpha}^{t}$. Let $\mathcal{S}_{i}$ denote the strategy set of player $i$. We write $\mathcal{S}_{S}=\times_{i \in S} \mathcal{S}_{i}$ for $S \subseteq N$. If $S=N \backslash\{i\}$ for some $i \in N$, we write $\mathcal{S}_{-i}$.

Definition 6.4. Let $\Gamma=(\mathbf{v}, \mathbf{u})$ be a dynamic TU-game, and let $\Phi$ be a value on $\mathcal{G}^{N}$. We define the non-cooperative game $G_{1}(\Gamma, \Phi)=\left(\mathcal{S}_{1}^{1}, \ldots, \mathcal{S}_{n}^{1} ; \tilde{u}_{1}, \ldots, \tilde{u}_{n}\right)$ as follows: For all $i \in N$ let the 
- strategy set $\mathcal{S}_{i}^{1}$ be the collection of vectors $\alpha_{i} \in \mathbb{R}^{T}$ such that

1. $v^{t}(i)+\alpha_{i}^{t} \geq 0$ for all $t \in\{1, \ldots, T\}$

2. $\sum_{t=1}^{T} \alpha_{i}^{t}=0$.

- utility function $\tilde{u}_{i}: \mathcal{S}_{N}^{1} \rightarrow \mathbb{R}$ such that

$$
\tilde{u}_{i}\left(\alpha_{1}, \ldots, \alpha_{n}\right)=u_{i}\left(\Phi_{i}\left(\left(v_{1, \alpha}^{t}\right)_{t=1}^{T}\right)\right) .
$$

with

$$
v_{1, \alpha}^{t}:=v^{t}+\sum_{j=1}^{n} \alpha_{j}^{t} 1_{j} .
$$

The definition of $G_{1}(\Gamma, \Phi)$ reflects the idea that individuals can transfer their individual worth among periods, while the worth of non-singleton coalitions remains untouched. The first condition on the strategies of players guarantees that $v_{\alpha}^{t}(i) \geq 0$ for all $t \in$ $\{1, \ldots, T\}$. The main idea behind this condition is that agents cannot borrow money from a third party (or if they can, then they already did so before the game is played). The second condition ensures that if agents withdraw worth from some period, then exactly this amount must be redistributed to other periods. Basically, this means that making transfers is not costly, nor does it generate additional worth. ${ }^{7}$ We presuppose these properties in all three transfer systems. Recall the example of the group of researchers from the introduction. In this example, we argued that the researcher might transfer her effort she is investing to some of the papers among periods. However, what we actually model is that agents can redistribute their individual worth among periods. We make the assumption that the characteristic function is common knowledge and, hence, when shifting effort - or whatever resource is considered in the underlying problemfrom one period to the other, the agent knows exactly how this affects the characteristic function of the corresponding stage games. With this model we cover a wider range of dynamic TU-games, as otherwise we had to consider different transfer systems for different underlying problems. Moreover, we argued that an agent should be able to relocate, not only her individual worth, but also some part of the worth of the coalitions that contain her. The next two transfer systems model the idea that individual transfers might also affect coalitions in different ways. Which transfer system is more suitable depends, of course, on the particular problem considered. Under the second transfer system we assume that the change of a coalition's worth equals the sum of the changes of the individual worths of its contained players. This restricts possible transfers of players in a natural way. For a given coalition $S$ the amount borrowed by individuals in $\mathrm{S}$ at period $t$ should not exceed $v^{t}(S)$. We are aware that this transfer system is limited in

\footnotetext{
${ }^{7}$ Note however, that by making transfers the payoff streams might change.
} 
its application to only some classes of TU-games, however, we want to outline it for completeness.

Definition 6.5. Let $\Gamma=(\mathbf{v}, \mathbf{u})$ be a dynamic TU-game, and let $\Phi$ be a value on $\mathcal{G}^{N}$. We define the non-cooperative game $G_{2}(\Gamma, \Phi)=\left(\mathcal{S}_{1}^{2}, \ldots, \mathcal{S}_{n}^{2} ; \tilde{u}_{1}, \ldots, \tilde{u}_{n}\right)$ as follows: For all $i \in N$ let the

- strategy set $\mathcal{S}_{i}^{2}$ be the collection of vectors $\alpha_{i} \in \mathbb{R}^{T}$ such that

1. $v^{t}(S)+\sum_{j \in S} \alpha_{j}^{t} \geq 0$ for all $t \in\{1, \ldots, T\}$ and all $S \in \mathcal{N}$

2. $\sum_{t=1}^{T} \alpha_{i}^{t}=0$.

- utility function $\tilde{u}_{i}: \mathcal{S}_{N}^{2} \rightarrow \mathbb{R}$ such that

$$
\tilde{u}_{i}\left(\alpha_{1}, \ldots, \alpha_{n}\right)=u_{i}\left(\Phi_{i}\left(\left(v_{2, \alpha}^{t}\right)_{t=1}^{T}\right)\right) .
$$

with

$$
v_{k, \alpha}^{t}:=v^{t}+\sum_{j=1}^{n} \alpha_{j}^{t} 2_{j} .
$$

The third transfer system allows agents to transfer their contribution to coalitions, provided that the amount borrowed by all agents in a coalition does not excel the worth of the coalition. In order to give a formal definition, we need additional notation. Let $\mathcal{N}=2^{N} \backslash\{\emptyset\}$ and $S^{i}=\{S \subseteq N \mid i \in S\}$. Moreover, for $S \in \mathcal{N}$, let

$$
c_{S}^{t}(i):=v^{t}(S)-v^{t}(S \backslash\{i\})
$$

denote $i$ 's contribution to coalition $S \backslash\{i\}$ at period $t \in\{1, \ldots, T\}$.

Definition 6.6. Let $\Gamma=(\mathbf{v}, \mathbf{u})$ be a dynamic TU-game, and let $\Phi$ be a value on $\mathcal{G}^{N}$. We define the non-cooperative game $G_{3}(\Gamma, \Phi)=\left(\mathcal{S}_{1}^{3}, \ldots, \mathcal{S}_{n}^{3} ; \tilde{u}_{1}, \ldots, \tilde{u}_{n}\right)$ as follows: For all $i \in N$ let the

- strategy set $\mathcal{S}_{i}^{3}$ be the collection of vectors $\alpha_{i} \in \mathbb{R}^{T \times|\mathcal{N}|}$ such that

1. $c_{S}^{t}(i)+\alpha_{i, S}^{t} \geq 0$ for all $t \in\{1, \ldots T\}$ and all $S \in \mathcal{N}$

2. $v^{t}(S)+\sum_{j \in S} \alpha_{j, S}^{t} \geq 0$ for all $t \in\{1, \ldots T\}$ and all $S \in \mathcal{N}$

3. $\sum_{t=1}^{T} \alpha_{i, S}^{t}=0$ for all $S \in \mathcal{N}$.

- utility function $\tilde{u}_{i}: \mathcal{S}_{N}^{3} \rightarrow \mathbb{R}$ such that

$$
\tilde{u}_{i}\left(\alpha_{1}, \ldots, \alpha_{n}\right)=u_{i}\left(\Phi_{i}\left(\left(v_{3, \alpha}^{t}\right)_{t=1}^{T}\right)\right) .
$$


with

$$
v_{3, \alpha}^{t}:=v^{t}+\sum_{S \in \mathcal{N}}\left(\sum_{i \in N} \alpha_{i, S}^{t} 1_{S}\right) .
$$

Observe that the first and the second transfer system can be considered instances of the third transfer system. Example 6.1 illustrates this.

Example 6.1. Let $v^{1}(1)=v^{1}(2)=1$ and $v^{1}(12)=2$ and let $v^{2}$ be the zero game. The following matrices represent (some) possible transfers of agent 1.

$$
\alpha_{1}=\left(\begin{array}{ccc}
-1 & 0 & 0 \\
1 & 0 & 0
\end{array}\right) ; \alpha_{1}^{\prime}=\left(\begin{array}{ccc}
-1 & 0 & -1 \\
1 & 0 & 1
\end{array}\right) ; \alpha_{1}^{\prime \prime}=\left(\begin{array}{ccc}
-\frac{1}{2} & 0 & -1 \\
\frac{1}{2} & 0 & 1
\end{array}\right)
$$

The first row in each matrix indicates which transfers player one makes in period one from or to $v^{1}(1), v^{1}(2)$, and $v^{1}(12)$ in that order. Obviously the contribution to a coalition of which a player is not part of is equal to zero. Analogously, the second row indicates transfers in period 2. Matrix $\alpha_{1}$ represents transfers only with respect to $v(1)$ and hence would also be possible under the first transfer system. The matrix $\alpha_{1}^{\prime}$ represents a transfer that affects $v(1)$ in the same way as $v(12)$ and hence is also possible under transfer system 2. The third matrix however, is not covered neither by first, nor the second transfer system.

\subsubsection{Feasibility constraints}

Let $k=1,2,3$ and note that $\mathcal{S}_{i}^{k} \neq \emptyset$ as agents can always make zero transfers under all three transfer systems. Hence, the strategy set of a player is never empty. However there might arise several feasibility problems. The following examples illustrate them.

Example 6.2. Let $N=\{1,2\}$ and $T=2$. Consider the sequence of $T U$-games $v^{1}(1)=$ $v^{1}(2)=v^{1}(12)=2$ and $v^{2}(1)=v^{2}(2)=v^{2}(12)=1$. Further, let $\Phi$ be the Shapley value $S h$ and $u_{i}\left(S h_{i}\left(v^{1}\right), S h_{i}\left(v^{2}\right)\right)=\sqrt{S h_{i}\left(v^{1}\right)}+\sqrt{S h_{i}\left(v^{2}\right)}$ be the intertemporal utility function for each $i \in N$. Note that the Shapley value for the initial game is defined and positive for each agent in each period. Suppose the first transfer system is considered and transfers are $\alpha_{1}=(-2,2)$ and $\alpha_{2}=(0,0)$. The resulting dynamic game is then as follows: $v_{1, \alpha}^{1}(1)=0, v_{1, \alpha}^{1}(2)=2, v_{1, \alpha}^{1}(12)=2$ while $v_{1, \alpha}^{2}(1)=3, v_{1, \alpha}^{2}(2)=$ $1, v_{1, \alpha}^{2}(12)=1$. The Shapley values of the resulting games are $\operatorname{Sh}\left(v_{1, \alpha}^{1}\right)=(0,2)$ and $\operatorname{Sh}\left(v_{1, \alpha}^{2}\right)=\left(\frac{3}{2},-\frac{1}{2}\right)$. In this case, $\left(S h_{2}\left(v_{1, \alpha}^{t}\right)\right)_{t=1}^{2}$ is not in the domain of the intertemporal utility function of agent 2 .

Example 6.3. Let $N=\{1,2,3\}$ and $T=2$. Consider the sequence of TU-games $v^{t}(1)=$ $1, v^{t}(2)=v^{t}(3)=v^{t}(12)=v^{t}(13)=v^{t}(23)=0, v^{t}(123)=1$ for $t=1,2$. Further, let 
$\Phi$ be the $\tau$-value. Observe that in each period the $\tau$-value is well defined $\tau\left(v^{t}\right)=(1,0,0)$ for $t=1,2$. Suppose the first transfer system and let $\alpha_{1}=(-1,1)$. The resulting dynamic TU-game is then $v_{1, \alpha}^{1}(1)=v_{1, \alpha}^{1}(2)=v_{1, \alpha}^{1}(3)=v_{1, \alpha}^{1}(12)=v_{1, \alpha}^{1}(13)=v_{1, \alpha}^{1}(23)=$ $0, v_{1, \alpha}^{1}(123)=1$ and $v_{1, \alpha}^{2}(1)=2, v_{1, \alpha}^{2}(2)=v_{1, \alpha}^{2}(3)=v_{1, \alpha}^{2}(12)=v_{1, \alpha}^{2}(13)=v_{1, \alpha}^{2}(23)=$ $0, v_{1, \alpha}^{2}(123)=1$. However, the $\tau$-value is not defined for $v_{1, \alpha}^{2}$.

Hence, we have to guarantee that $\tilde{u}_{i}(\alpha)$ and $\Phi_{i}\left(v_{\alpha}\right)$ are well defined for all $i \in N$ and all $\alpha \in \mathcal{S}_{N}^{k}$ for $k=1,2,3$. One possible approach is to put more constraints on the strategy set of players, but these constraints would obviously very much depend on the utility function, as well as on the solution concept applied. Hence, for each particular problem, players' strategy set must be redefined. We take a different approach by defining $k$-feasible tuple.

Definition 6.7. Let $k \in\{1,2,3\}$ and fix a value $\Phi$ for $\mathcal{G}^{N}$. A tuple $(\mathbf{u}, \mathbf{v})$ is k-feasible for $G_{k}(\Gamma, \Phi)$, if for all $i \in N$, all $\alpha \in \mathcal{S}_{N}^{k}$ and $t \in\{1, \ldots, T\}$

1. $\Phi$ is well defined for all $v_{k, \alpha}^{t}$ and

2. $\left(\Phi_{i}\left(v_{k, \alpha}^{t}\right)\right)_{t=1}^{T}$ is in the domain of $u_{i}$.

The above definition ensures that $\tilde{u}_{i}$ is well defined for all $i \in N$ and $\alpha \in \mathcal{S}_{N}^{k}$ given $\Phi$. In the sequel of the chapter all results are with respect to feasible tuples for the corresponding non-cooperative game.

The main goal lies in finding Nash equilibria in $G_{k}(\Gamma, \Phi)$ for $k \in\{1,2,3\}$. Usually, in non-cooperative games players' feasible strategies are independent of the strategies chosen by the other players. However, due to the assumption that $v^{t}(S) \geq 0$ for all $S \subseteq N$ and all $t=1, \ldots, T$ we might have constraints across strategies of players. The following example illustrates this.

Example 6.4. Let $N=\{1,2\}$ and $T=2$. Consider the sequence of TU-games $v^{1}(1)=$ $v^{1}(2)=0, v^{1}(12)=2$ and $v^{2}(1)=v^{2}(2)=v^{2}(12)=1$, and consider the third transfer system. Figure 12 shows the possible transfers in the first period for player one to coalition $\{1,2\}$, indicated by I and II, respectively. ${ }^{8}$ The set of feasible strategy combinations is colored grey. Observe that for example in period one the strategy combination where both players transfer away all their marginal contribution to coalition $\{1,2\}$ is infeasible, as this would result in $v_{\alpha}^{1}(12)=-2$ which contradicts our assumption that $v^{t}(S) \geq 0$ for all $t=1, \ldots, T$ and all $S \subseteq N$.

\footnotetext{
${ }^{8}$ Due to symmetry the the possible transfers of player 2 in period 1 restricted to coalition $\{1,2\}$ are identical.
} 


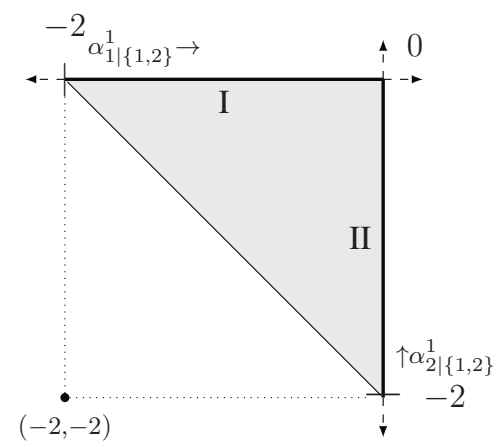

Figure 12: Constraints across players' strategies

This problem arises when considering the second and the third, but not the first transfer system. We transform the non-cooperative games defined above into games played in a constraint environment and then apply Theorem 1 of Morales \& Vermeulen (2009) in order to prove existence of Nash equilibria. ${ }^{9}$ Let us now define best responses. Consider player $i$ and let $k=1,2,3$. Given a strategy combination $\alpha \in \mathcal{S}_{N}^{k}$ the set of feasible responses of player $i$ is

$$
\mathcal{D}_{i}^{k}(\alpha):=\left\{\alpha^{\prime} \in \mathcal{S}_{N}^{k} \mid \alpha_{j}^{\prime}=\alpha_{j} \text { for all } j \neq i\right\}
$$

The set of best responses of player $i$ is

$$
B R_{i}^{k}(\alpha):=\left\{\beta \in \mathcal{D}_{i}^{k}(\alpha) \mid \tilde{u}_{i}(\beta) \geq \tilde{u}_{i}(\gamma) \text { for all } \gamma \in D_{i}^{k}(\alpha)\right\}
$$

Definition 6.8. Let $k=1,2,3$. A strategy profile $\alpha \in \mathcal{S}_{N}^{k}$ is called a Nash equilibrium of the game in a constrained environment $G_{k}$ if for all $i \in N$ we have that $\alpha$ is an element of $B R_{i}^{k}(\alpha)$.

Let $k=1,2,3$. For $G_{k}=\left(\mathcal{S}_{N}^{k},\left(B R_{i}^{k}\right)_{i \in N}\right)$ to be a game played in a constrained environment, it remains to show that the graph of $B R_{i}^{k}$ is a closed subset of $\mathcal{S}_{N}^{k} \times \mathcal{S}_{N}^{k}$, and that for each $\alpha \in \mathcal{S}_{N}^{k}, B R_{i}^{k}(\alpha)$ is a non-empty subset of $\mathcal{D}_{i}^{k}(\alpha)$.

Lemma 6.1. Let $k=1,2,3$, and let $\Phi_{i}$ and $u_{i}$ be continuous for all $i \in N$. Then the graph of $B R_{i}^{k}$ is a closed subset of $\mathcal{S}_{N}^{k} \times \mathcal{S}_{N}^{k}$.

Proof. Suppose $\beta^{n} \in B R_{i}^{k}\left(\alpha^{n}\right)$ for all $n$ and that $\left(\left(\beta^{n}\right),\left(\alpha^{n}\right)\right) \rightarrow((\beta),(\alpha))$ as $n \rightarrow \infty$. Take any point $(\gamma) \in \mathcal{D}_{i}^{k}(\alpha)$. Notice that $\mathcal{S}_{N}^{k}$ is polyhedral. We know, due to for instance

\footnotetext{
${ }^{9}$ If there are no constraints across players' strategies, as it is the case in $G_{1}(\Gamma, \Phi)$, this approach can still be used. The game then simplifies to a conventional normal form game.
} 
Cook et al. (1986), that there exist points $\left(\gamma^{n}\right) \in \mathcal{D}_{i}^{k}\left(\alpha^{n}\right)$ that converge to $\gamma$ as $n \rightarrow \infty$. However, as $\left(\beta^{n}\right)$ is an element of $B R_{i}\left(\alpha^{n}\right)$ we have for all $n$ that $\tilde{u}_{i}\left(\beta^{n}\right) \geq \tilde{u}_{i}\left(\gamma^{n}\right)$. By continuity, $\tilde{u}_{i}(\beta) \geq \tilde{u}_{i}(\gamma)$.

Lemma 6.2. Let $k=1,2,3$, and let $\Phi$ be continuous and $u_{i}$ be continuous for all $i \in N$. Then for each $\alpha \in \mathcal{S}_{N}^{k}, B R_{i}^{k}(\alpha)$ is a non-empty subset of $\mathcal{D}_{i}^{k}(\alpha)$.

Proof. Since $u_{i}$ is continuous for all $i \in N$ and $\Phi$ is continuous, we have that $\tilde{u}_{i}$ is continuous for every $i \in N$. By definition

$$
B R_{i}^{k}(\alpha)=\underset{\beta \in \mathcal{D}_{i}^{k}(\alpha)}{\arg \max } \tilde{u}_{i}(\beta),
$$

where $\mathcal{D}_{i}^{k}(\alpha)$ is non-empty and compact. By Weierstrass Extreme Value Theorem we have that $B R_{i}(\alpha)$ is non-empty.

\subsubsection{Existence of Nash equilibrium}

We are now going to show that the best response correspondence of the above games is convex-valued. In order to do so, we first introduce a little more notation.

Definition 6.9 (transfer-1-concavity, transfer-2-concavity). Let $k \in\{1,2\}$. We say $\Phi$ is transfer-k-concave if

$$
\Phi\left(\lambda(v+w)+(1-\lambda)\left(v+w^{\prime}\right)\right) \geq \lambda \Phi(v+w)+(1-\lambda) \Phi\left(v+w^{\prime}\right)
$$

for all $\lambda \in(0,1)$ and all $v, w, w^{\prime} \in \mathcal{G}^{N}$ such that $w=\sum_{i=1}^{n} w(i) \mathbf{k}_{i}$ and $w^{\prime}=\sum_{i=1}^{n} w^{\prime}(i) \mathbf{k}_{i}$ with $(w(1), \ldots, w(n)) \in \mathbb{R}^{N}$ and $\left(w^{\prime}(1), \ldots, w^{\prime}(n)\right) \in \mathbb{R}^{N}$.

Definition 6.10 (transfer-3-concavity). We say $\Phi$ is transfer-3-concave if

$$
\Phi\left(\lambda(v+w)+(1-\lambda)\left(v+w^{\prime}\right)\right) \geq \lambda \Phi(v+w)+(1-\lambda) \Phi\left(v+w^{\prime}\right)
$$

for all $\lambda \in(0,1)$ and all $v, w, w^{\prime} \in \mathcal{G}^{N}$.

Specifically, we require the solution to be concave on the class of all games that can be generated by transfers made in accordance with the particular transfer system.

It is easy to see that transfer-1- and transfer-2-concavity are weaker conditions than concavity. Although transfer-3-concavity is equivalent to concavity we refer to it as transfer-3-concavity for purpose of convenience throughout this section of the chapter.

We are now able to prove that the best response correspondence is convex valued. 
Lemma 6.3. Let $k \in\{1,2,3\}$ and let $G_{k}$ be a game played in a constrained environment. Let $\Phi$ be transfer- $k$-concave and $u_{i}$ be increasing and quasiconcave for every $i \in N$. Then $B R_{i}^{k}(\alpha)$ is convex for all $i \in N$ and every $\alpha \in \mathcal{S}_{N}^{k}$.

Proof. Fix any arbitrary $\beta, \gamma \in B R_{i}(\alpha)$. Then

$$
\begin{aligned}
& \tilde{u}_{i}(\beta) \geq \tilde{u}_{i}(\sigma) \text { for all } \sigma \in \mathcal{D}_{i}^{k}(\alpha) \\
& \tilde{u}_{i}(\gamma) \geq \tilde{u}_{i}(\sigma) \text { for all } \sigma \in \mathcal{D}_{i}^{k}(\alpha) .
\end{aligned}
$$

We show that $\tilde{u}_{i}(\lambda \beta+(1-\lambda) \gamma) \geq \tilde{u}_{i}(\sigma)$ for all $\sigma \in \mathcal{D}_{i}^{k}(\alpha)$ and all $\lambda \in(0,1)$.

$$
\begin{aligned}
& \tilde{u}_{i}(\lambda \beta+(1-\lambda) \gamma) \\
& =u_{i}\left(\Phi_{i}\left(v_{k,(\lambda \beta+(1-\lambda) \gamma)}^{t}\right)_{t=1}^{T}\right) \\
& =u_{i}\left(\Phi_{i}\left(\lambda v_{k, \beta}^{t}+(1-\lambda) v_{k, \gamma}^{t}\right)_{t=1}^{T}\right) \\
& \geq u_{i}\left(\lambda\left(\Phi_{i}\left(v_{k, \beta}^{1}\right)_{t=1}^{T}\right)+(1-\lambda)\left(\Phi_{i}\left(v_{k, \gamma}^{1}\right)_{t=1}^{T}\right)\right) \\
& \geq \min \left\{u_{i}\left(\Phi_{i}\left(v_{k, \beta}^{t}\right)_{t=1}^{T}\right), u_{i}\left(\Phi_{i}\left(v_{k, \gamma}^{t}\right)_{t=1}^{T}\right)\right. \\
& =\min \left\{\tilde{u}_{i}(\beta), \tilde{u}_{i}(\gamma)\right\} .
\end{aligned}
$$

The first equality follows from the definition of $u_{i}$ and the first inequality follows from transfer-k-concavity of the solution and the fact that $u_{i}$ is increasing. The second inequality follows from quasiconcavity of $u_{i}$. The last equality follows from the definition of $\tilde{u}_{i}$. Hence we conclude that $\tilde{u}_{i}(\lambda \beta+(1-\lambda) \gamma) \geq \tilde{u}_{i}(\sigma)$ for all $i \in N$. Thus $B R_{i}^{k}(\alpha)$ is a convex set for all $i \in N$ and $\alpha \in \mathcal{S}_{N}^{k}$.

Theorem 6.2. Let $k=1,2,3$, let $\Gamma=(\mathbf{u}, \mathbf{v})$ be a dynamic $T U$-game and let $G_{k}$ be a game played in a constrained environment as defined above. Let $F$ be continuous and transfer- $k$-concave and $u_{i}$ be increasing, quasiconcave and continuous for all $i \in N$. Then a Nash equilibrium exists in $G_{k}$.

Proof. This follows immediately from Lemma 6.1, Lemma 6.2 and Lemma 6.3 together with Theorem 6.1.

In what follows we will provide sufficient conditions for transfer-k-concavity with $k=1,2,3$. We then relate our results to some well-known point-wise solutions.

Lemma 6.4. Let $\Phi$ be linear. Then it satisfies transfer- $k$-concavity for $k=1,2,3$.

Proof. Let $\lambda \in(0,1)$. Let $v=\lambda(u+w)+(1-\lambda)\left(u+w^{\prime}\right) \in \mathcal{G}^{N}$ where $w=\sum_{i=1}^{n} w(i) 1_{i}$ with $(w(1), \ldots, w(n)) \in \mathbb{R}^{N}$ and $w^{\prime}=\sum_{i=1}^{n} w^{\prime}(i) 1_{i}$ with $\left(w^{\prime}(1), \ldots, w^{\prime}(n)\right) \in \mathbb{R}^{N}$. We 
have to show that $\Phi_{i}\left(\lambda(u+w)+(1-\lambda)\left(u+w^{\prime}\right)\right) \geq \lambda \Phi_{i}(u+w)+(1-\lambda) \Phi_{i}\left(u+w^{\prime}\right)$. Fix $i \in N$. We have $\Phi_{i}(v)=\Phi_{i}\left(\lambda(u+w)+(1-\lambda)\left(u+w^{\prime}\right)\right)=\Phi_{i}(\lambda u+(1-\lambda) u+$ $\left.\lambda w+(1-\lambda) w^{\prime}\right)$. By linearity of $\Phi$ this is equal to

$$
\begin{aligned}
& \Phi_{i}(u)+\lambda \Phi_{i}(w)+(1-\lambda) \Phi_{i}\left(w^{\prime}\right) \\
& =\lambda \Phi_{i}(u)+(1-\lambda) \Phi_{i}(u)+\lambda \Phi_{i}(w)+(1-\lambda) \Phi_{i}\left(w^{\prime}\right) \\
& =\lambda\left(\Phi_{i}(u)+\Phi_{i}(w)\right)+(1-\lambda)\left(\Phi_{i}(u)+\Phi_{i}\left(w^{\prime}\right)\right) .
\end{aligned}
$$

Since this is true for all $i \in N$ we conclude that transfer-1-concavity is satisfied. The proof for transfer-2- and transfer-3-concavity are analogous and therefore omitted.

Remark 6.1. Notice that instead of linearity of $\Phi$, we could also require that $\Phi$ is additive and continuous in only $v(i)$ for all $i \in N$ or $\Phi$ is additive and increasing in only $v(i)$ for all $i \in N$. Both combinations of conditions are weaker requirements than linearity. However, in order to construct solutions that are additive but not linear one needs the axiom of choice. As we apply solutions that are already defined in the literature, and hence all additive solutions satisfy linearity, we focus on linear solutions in the sequel.

We derive the following corollary from Lemma 6.4 and Theorem 6.2.

Corollary 6.1. Let $\Gamma=(\mathbf{v}, \mathbf{u})$ be a dynamic $T U$-game and let $\Phi$ be linear. Let $u_{i}$ be continuous, increasing and quasiconcave for every $i \in N$. Then there exists a Nash equilibrium in $G_{1}$.

Examples for solutions that satisfy linearity are displayed in Table 2.

We have already identified linearity as a sufficient condition for transfer-2-concavity, however, also relative invariance under strategic equivalence turns out to be sufficient for transfer-2-concavity.

Lemma 6.5. If $\Phi$ is relatively invariant under strategic equivalence and $\Phi(z)=0$ then it satisfies transfer-2-concavity.

Proof. Let $\Phi$ be relatively invariant under strategic equivalence and $\Phi(z)=0$. Let $\lambda \in(0,1)$. Let $v=\lambda(u+w)+(1-\lambda)\left(u+w^{\prime}\right) \in \mathcal{G}^{N}$ where $w=\sum_{i=1}^{n} w(i) 2_{i}$ with $(w(1), \ldots, w(n)) \in \mathbb{R}^{N}$ and $w^{\prime}=\sum_{i=1}^{n} w^{\prime}(i) 2_{i}$ with $\left(w^{\prime}(1), \ldots, w^{\prime}(n)\right) \in \mathbb{R}^{N}$. We have to show that $\Phi_{i}(\lambda(u+w)+(1-\lambda)(u+w)) \geq \lambda \Phi_{i}(u+w)+(1-\lambda) \Phi_{i}(u+w)$. Fix $i \in N$. We have $\Phi_{i}(v)=\Phi_{i}\left(\lambda(u+w)+(1-\lambda)\left(u+w^{\prime}\right)\right)=\Phi_{i}(\lambda u+(1-\lambda) u+\lambda w+$ $\left.(1-\lambda) w^{\prime}\right)=\Phi_{i}\left(u+\lambda w+(1-\lambda) w^{\prime}\right)$. By relative invariance under strategic equivalence this is equal to $\Phi_{i}(u)+\lambda w(i)+(1-\lambda) w^{\prime}(i)=\lambda \Phi_{i}(u)+(1-\lambda) \Phi_{i}(u)+\lambda w(i)+(1-$ $\lambda) w^{\prime}(i)=\lambda\left(\Phi_{i}(u)+w(i)\right)+(1-\lambda)\left(\Phi_{i}(u)+w^{\prime}(i)\right)$. By relative invariance under strategic equivalence this equals $\lambda \Phi_{i}(u+w)+(1-\lambda) \Phi_{i}\left(u+w^{\prime}\right)$. Since this is true for all $i \in N$ we conclude that transfer-2-concavity is satisfied. 


\begin{tabular}{ll}
$\alpha_{1}$ & $\tau\left(v+\alpha_{1} 1_{1}\right)$ \\
\hline$\alpha_{1}=0$ & $\tau\left(v+01_{1}\right)=\left(\frac{25}{13}, \frac{28}{13}, \frac{25}{13}\right)$ \\
$\alpha_{1}=\frac{1}{2}$ & $\tau\left(v+\frac{1}{2} 1_{1}\right)=\left(\frac{53}{25}, \frac{52}{25}, \frac{45}{25}\right)$ \\
$\alpha_{1}=1$ & $\tau\left(v+11_{1}\right)=\left(\frac{7}{3}, 2, \frac{5}{3}\right)$ \\
\hline
\end{tabular}

Table 3: $\tau$-value for different transfers $\alpha_{1}$

We derive the following corollary from Theorem 6.2 and Lemma 6.5.

Corollary 6.2. Let $\Gamma=(\mathbf{v}, \mathbf{u})$ be a dynamic game and let $\Phi$ satisfy relative invariance under strategic equivalence and $\Phi(z)=0$. Let $u_{i}$ be continuous, increasing and quasiconcave for every $i \in N$. Then there exists a Nash equilibrium in $G_{2}$.

The Shapley value, the nucleolus and the prenucleolus, the $\tau$-value, as well as the consensus value are solutions that satisfy relative invariance under strategic equivalence.

Notice that relative invariance under strategic equivalence is not implied by linearity and hence is not weaker than it.

Example 6.5. Let $\Phi$ be the egalitarian Shapley value with $\lambda=\frac{1}{2}$. Let $N=\{1,2,3\}$ and $v$ be the additive game generated by $(0,1,2)$. It is sufficient to show that $S h^{\frac{1}{2}}(v) \neq(0,1,2)$. We have that $\operatorname{Sh}(v)=(0,1,2)$ and $E D(v)=(1,1,1)$, hence $\operatorname{Sh}^{\frac{1}{2}}(v)=\left(\frac{1}{2}, 1, \frac{3}{2}\right)$.

The following example illustrates that the $\tau$-value is not concave under the first transfer system. Hence it shows that relative invariance of strategic equivalence is not sufficient for transfer-1-concavity.

Example 6.6. Consider the game $v$ where $v(1)=0, v(2)=1, v(3)=0, v(12)=v(23)=$ $1, v(13)=2$ and $v(123)=6$. Suppose that the first transfer system is used and only player one makes transfers. We compute the $\tau$-value $\tau\left(v+\alpha_{1} 1_{1}\right)$ for different values $\alpha_{1}$. The results are displayed in Table 3.

Now let $\lambda=\frac{1}{2}$ and take $\beta_{1}=0$ and $\gamma_{1}=1$. Then $\tau_{1}\left(\lambda\left(v+\beta_{1} 1_{1}\right)+(1-\lambda)(v+\right.$ $\left.\left.\gamma_{1} 1_{1}\right)\right)=\frac{53}{25}$. However, $\lambda \tau_{1}\left(v+\beta_{1} 1_{1}\right)+(1-\lambda) \tau_{1}\left(v+\gamma_{1} 1_{1}\right)=\frac{1}{2} \tau_{1}\left(v+01_{1}\right)+\frac{1}{2} \tau_{1}(v+$ $\left.1_{1}\right)=\frac{83}{39}$. Since $\frac{53}{25} \nsucceq \frac{83}{39}$ we conclude that the $\tau$-value is not concave under the first transfer system. ${ }^{10}$

The next example illustrates, that a Nash equilibrium need not exist in $G_{1} \cdot{ }^{11}$

\footnotetext{
${ }^{10} \mathrm{~A}$ similar example is given in Berden (2007) for the nucleolus.

${ }^{11}$ The example is taken and adapted from Berden (2007). As there are no constraints across players strategies, we slightly deviate from the notation for games played in a constraint environment, for the purpose of display.
} 


\begin{tabular}{ll} 
value of $\beta^{1}$ & $\tilde{u}_{1}(\alpha, \beta)$ \\
\hline \hline$\beta^{1}=1$ if $\alpha^{1} \in\left[0, \frac{1}{2}\right]$ & $4 \frac{1}{3}+\frac{1}{12} \beta^{1}-\frac{1}{6} \alpha^{1}$ \\
$\beta^{1}=1$ if $\alpha^{1} \in\left[\frac{1}{2}, 1\right]$ & $4 \frac{1}{3}$ \\
$\beta^{1} \in(0,1)$ if $\alpha^{1} \in\left[0, \frac{1}{2} \beta^{1}\right]$ & $4 \frac{1}{3}+\frac{1}{12} \beta^{1}-\frac{1}{6} \alpha^{1}$ \\
$\beta^{1} \in(0,1)$ if $\alpha^{1} \in\left[\frac{1}{2} \beta^{1}, \frac{1}{2} \beta^{1}+\frac{1}{2}\right]$ & $4 \frac{1}{3}$ \\
$\beta^{1} \in(0,1)$ if $\alpha^{1} \in\left[\frac{1}{2} \beta^{1}+\frac{1}{2}, 1\right]$ & $4 \frac{1}{3}-\frac{1}{12} \beta^{1}+\frac{1}{6} \alpha^{1}$ \\
$\beta^{1}=0$ if $\alpha^{1} \in\left[0, \frac{1}{2}\right]$ & $4 \frac{1}{3}$ \\
$\beta^{1}=0$ if $\alpha^{1} \in\left[\frac{1}{2}, 1\right]$ & $4 \frac{1}{3}-\frac{1}{12} \beta^{1}+\frac{1}{6} \alpha^{1}$ \\
\hline
\end{tabular}

Table 4: Player 1's utility for different transfers $\alpha$ and $\beta$

\begin{tabular}{ll} 
value of $\alpha^{1}$ & $\tilde{u}_{2}(\alpha, \beta)$ \\
\hline \hline$\alpha^{1} \in\left[0, \frac{1}{2}\right]$ if $\beta^{1} \in\left[0,2 \alpha^{1}\right]$ & $4 \frac{1}{3}$ \\
$\alpha^{1} \in\left[0, \frac{1}{2}\right]$ if $\beta^{1} \in\left[2 \alpha^{1}, 1\right]$ & $4 \frac{1}{3}-\frac{1}{6} \beta^{1}+\frac{1}{3} \alpha^{1}$ \\
$\alpha^{1} \in\left[\frac{1}{2}, 1\right]$ if $\beta^{1} \in\left[0,2 \alpha^{1}-1\right]$ & $4 \frac{1}{3}+\frac{1}{6} \beta^{1}-\frac{1}{3} \alpha^{1}$ \\
$\alpha^{1} \in\left[\frac{1}{2}, 1\right]$ if $\beta^{1} \in\left[2 \alpha^{1}-1,1\right]$ & $4 \frac{1}{3}$ \\
\hline
\end{tabular}

Table 5: Player 2's utility for different transfers $\alpha$ and $\beta$

Example 6.7. Let $N=\{1,2,3\}$ and consider the following dynamic TU-game $\Gamma=$ $\left(v^{1}, v^{2}, u_{1}, u_{2}, u_{3}\right)$, the nucleolus $\nu$ and the first transfer system. The game $v^{1}$ is described by $v^{1}(1)=v^{1}(2)=v^{1}(3)=0, v^{1}(12)=v^{1}(23)=1, v^{1}(13)=2$ and $v^{1}(123)=6$. For $v^{2}$ we have $v^{2}(1)=v^{2}(2)=1, v^{2}(S)=v^{1}(S)$ for all $S \neq\{1\},\{2\}$. Observe that player 3 is just a strategic dummy player, while player 1 and 2 can make transfers among periods. The vectors $\alpha=\left(\alpha^{1}, \alpha^{2}\right) \in \mathcal{S}_{1}^{1}$ and $\beta=\left(\beta^{1}, \beta^{2}\right) \in \mathcal{S}_{2}^{1}$ contain all possible strategies resulting from transferring payoffs of player 1 and 2, respectively. Moreover, $\alpha^{1}+\alpha^{2}=\beta^{1}+\beta^{2}=0$ and $\alpha^{1}, \beta^{1} \in[0,1]$. Furthermore, let $u_{i}\left(\nu_{i}\left(v^{t}\right)_{t=1}^{T}\right)=\nu_{i}^{1}+\nu_{i}^{2}$ for all $i \in N$. Player 1's utility for any transfers $\left(\beta^{1},-\beta^{1}\right)$ of player 2 , is given in Table 4. Table 5 displays player 2's utility for different transfers $\left(\alpha^{1},-\alpha^{1}\right)$ of player 1. Table 6 shows the reaction functions of player 1 and 2. It contains player 1 's best response $\left(\alpha^{1 *},-\alpha^{1 *}\right)$ against any possible transfer $\left(\beta^{1},-\beta^{1}\right)$ of player 2 . Analogously, it shows player 2 's best response $\left(\beta^{1 *},-\beta^{1 *}\right)$ for every possible transfer $\left(\alpha^{1},-\alpha^{1}\right)$ of player 1 .

Figure 13 shows a plot of the best response sets of players 1 and 2, where player 1's best response set consists of parts I. a and I.b while player 2's best response set consists of part II. It is easy to see that there is no overlap in the best response sets of both players, hence no Nash equilibrium exists. 


\begin{tabular}{llll}
\multicolumn{2}{l}{ Reaction function player 1} & \multicolumn{2}{c}{ Reaction function player 2} \\
\hline \hline value of $\beta^{1}$ & best response $\alpha^{1 *}$ & value of $\alpha_{1}$ & best response $\beta^{1 *}$ \\
\hline$\beta^{1} \in\left[0, \frac{1}{2}\right]$ & $\alpha^{1 *}=1$ & $\alpha^{1} \in\left[0, \frac{1}{2}\right]$ & $\beta^{1 *} \in\left[0,2 \alpha^{1}\right]$ \\
$\beta^{1} \in\left[\frac{1}{2}, 1\right]$ & $\alpha^{1 *}=0$ & $\alpha^{1} \in\left[\frac{1}{2}, 1\right]$ & $\beta^{1 *} \in\left[2 \alpha^{1}-1,1\right]$ \\
\hline
\end{tabular}

Table 6: Best response functions of player 1 and 2

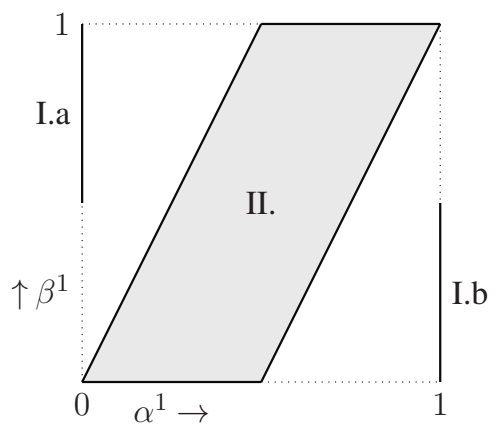

Figure 13: Non existence of Nash equilibrium

\subsubsection{Characterization of Nash equilibrium}

A profile of transfers is a Nash equilibrium if no player can increase her utility by reshuffling her transfers. Intuitively, this means that the marginal utilities of each player at each period should be equal. We provide a characterization of the Nash equilibria based on this intuition. In order to do so, we need a little more notation.

Let $v_{k, \alpha}^{t}(S)$ denote coalition $S$ 's worth in $v_{k, \alpha}^{t}$ for $k \in\{1,2,3\}$.

Theorem 6.3. Let $k \in\{1,2,3\}$. Let $\tilde{u}_{i}: \mathcal{S}_{N}^{k} \rightarrow \mathbb{R}$ be concave and such that the partial derivatives exist and are continuous.

1.

$$
\frac{\partial \tilde{u}_{i}}{\partial \alpha_{i}^{t}}(\alpha) \geq \frac{\partial \tilde{u}_{i}}{\alpha_{i}^{t^{\prime}}}(\alpha)
$$

for all $i \in N$ and $t, t^{\prime} \in\{1, \ldots, T\}$ such that $v_{1, \alpha}^{t}(i)>0$ if and only if $\alpha \in \mathcal{S}_{N}^{1}$ is a Nash equilibrium in $G_{1}$. 
2.

$$
\frac{\partial \tilde{u}_{i}}{\partial \alpha_{i}^{t}}(\alpha) \geq \frac{\partial \tilde{u}_{i}}{\alpha_{i}^{t^{\prime}}}(\alpha)
$$

for all $i \in N$, and $t, t^{\prime} \in\{1, \ldots, T\}$ such that $v_{2, \alpha}^{t}(S)>0$ if and only if $\alpha \in \mathcal{S}_{N}^{2}$ is a Nash equilibrium in $G_{2}$.

3.

$$
\frac{\partial \tilde{u}_{i}}{\partial \alpha_{i, S}^{t}}(\alpha) \geq \frac{\partial \tilde{u}_{i}}{\alpha_{i, S}^{t^{\prime}}}(\alpha)
$$

for all $i \in N, S \in S^{i}$ and $t, t^{\prime} \in\{1, \ldots, T\}$ such that $v_{3, \alpha}^{t}(S)>0$ if and only if $\alpha \in \mathcal{S}_{N}^{3}$ is a Nash equilibrium in $G_{3}$.

Proof. We show that if $\alpha$ is a Nash equilibrium then 11 is satisfied. Suppose it is not satisfied, i.e., there is $i \in N$ such that

$$
\frac{\partial \tilde{u}_{i}}{\partial \alpha_{i}^{t}}(\alpha)<\frac{\partial \tilde{u}_{i}}{\alpha_{i}^{t^{\prime}}}(\alpha)
$$

for some $t, t^{\prime} \in\{1, \ldots, T\}$ where $v_{k, \alpha}^{t}(i)>0$. Hence, a marginal increase in $\alpha_{i}^{t^{\prime}}$ results in a higher increase in payoff than a marginal increase in $\alpha_{i}^{t}$. But then, player $i$ could increase his utility by making a transfer from period $t$ to $t^{\prime}$, which contradicts the assumption that $\alpha$ is a Nash equilibrium. The reasoning for transfer systems two and three is analogous and the proof is therefore omitted.

Now we show that if 11 holds, then $\alpha$ is a Nash equilibrium. In order to prove that, we make use of the Karush-Kuhn-Tucker (KKT) conditions. We give a proof for $k=1$. The proofs for $k=2$ and $k=3$ are similar and therefore relegated to the Appendix.

For $k=1$, fix player $i \in N$ and let $\alpha \in \mathcal{S}_{N}^{k}$ such that $\alpha_{j} \in \mathcal{D}_{j}^{1}(\alpha)$ for all $j \in N \backslash\{i\}$. Then player $i$ faces the following maximization problem,

$$
\begin{aligned}
& \max _{\alpha_{i}:\left(\alpha_{i}, \alpha_{-i}\right) \in \mathcal{D}_{i}^{1}(\alpha)} \tilde{u}_{i}(\alpha) \\
& \text { s.t. } \\
& g_{t}(\alpha)=-v^{t}(i)-\alpha_{i}^{t} \leq 0 \text { for } t=1, \ldots T \\
& h(\alpha)=\sum_{t=1}^{T} \alpha_{i}^{t}=0 .
\end{aligned}
$$

Note first, that all inequality constraints are binding only in the case where $v^{t}(i)=0$ for all $t \in\{1, \ldots, T\}$. However, if this is the case, the unique strategy of player $i$ is to make 
zero transfers. Hence, there is nothing to prove. So, suppose the first $l<T$ inequality constraints are binding. We first show that the non degenerate constraint qualification is satisfied.

$$
\left(\begin{array}{ccc}
\frac{\partial g_{1}}{\partial \alpha_{i}^{1}}(\alpha) & \ldots & \frac{\partial g_{1}}{\partial \alpha_{i}^{T}}(\alpha) \\
\vdots & \ddots & \vdots \\
\frac{\partial g_{l}}{\partial \alpha_{i}^{1}}(\alpha) & \ldots & \frac{\partial g_{l}}{\partial \alpha_{i}^{T}}(\alpha) \\
\frac{\partial h}{\partial \alpha_{i}^{1}}(\alpha) & \ldots & \frac{\partial h}{\partial \alpha_{i}^{T}}(\alpha)
\end{array}\right)=\left(\begin{array}{cccccc}
-1 & 0 & \ldots & 0 & \ldots & 0 \\
0 & -1 & \ldots & 0 & \ldots & 0 \\
\vdots & & \ddots & \vdots & & \vdots \\
0 & 0 & \ldots & -1 & \ldots & 0 \\
1 & 1 & \ldots & 1 & \ldots & 1
\end{array}\right)
$$

The rank of the Jacobian of the binding constraints is $l+1$ and the non degenerate constraint qualification is satisfied. We aim to maximize the Lagrangian

$$
L\left(\alpha, \lambda_{1}, \ldots, \lambda_{T}, \mu\right)=\tilde{u}_{i}(\alpha)+\sum_{t=1}^{T} \lambda_{t}\left[v^{t}(i)+\alpha_{i}^{t}\right]-\mu\left[\sum_{t=1}^{T} \alpha_{i}^{t}\right]
$$

The KKT conditions are:

1. $\frac{\partial L}{\partial \alpha_{i}^{t}}(\alpha)=0$ for $t=1, \ldots, T$

2. $\lambda_{t}\left(v^{t}(i)+\alpha_{i}^{t}\right)=0$ for $t=1, \ldots, T$

3. $\lambda_{t} \geq 0$ for $t=1, \ldots, T$

4. $v^{t}(i)+\alpha_{i}^{t} \geq 0$

5. $\sum_{t=1}^{T} \alpha_{i}^{t}=0$

Observe that the second KKT condition is satisfied if $\lambda_{t}=0$ or $\alpha_{i}^{t}=-v^{t}(i)$. Suppose $\lambda_{t}=0$ for some $t \in\{1, \ldots, T\}$. Then by the first KKT condition:

$$
\frac{\partial L}{\partial \alpha_{i}^{t}}(\alpha)=\frac{\partial \tilde{u}_{i}}{\partial \alpha_{i}^{t}}(\alpha)+\lambda_{t}-\mu=0
$$

Hence,

$$
\mu=\frac{\partial \tilde{u}_{i}}{\partial \alpha_{i}^{t}}(\alpha)
$$

This implies $\frac{\partial \tilde{u}_{i}}{\partial \alpha_{i}^{t}}(\alpha)=\frac{\partial \tilde{u}_{i}}{\partial \alpha_{i}^{t^{\prime}}}(\alpha)$ for all $t, t^{\prime} \in\{1, \ldots T\}$ with $\lambda_{t}=0$ and $\lambda_{t}^{\prime}=0$. Suppose $\alpha_{i}^{t^{\prime \prime}}=-v^{t^{\prime \prime}}(i)$ for some $t^{\prime \prime} \in\{1, \ldots T\}$. Then by the first KKT condition:

$$
\mu=\frac{\partial \tilde{u}_{i}}{\partial \alpha_{i}^{t^{\prime \prime}}}(\alpha)+\lambda_{t^{\prime \prime}}
$$


Suppose $\lambda_{t^{\prime \prime}} \geq 0$. Then from 15 and 16 it follows

$$
\frac{\partial \tilde{u}_{i}}{\partial \alpha_{i}^{t}}(\alpha)=\frac{\partial \tilde{u}_{i}}{\partial \alpha_{i}^{t^{\prime \prime}}}(\alpha)+\lambda_{t^{\prime \prime}}
$$

for $t \in\{1, \ldots T\}$ such that $\lambda_{t}=0$. By concavity of $\tilde{u}_{i}$ and the third KKT condition

$$
\frac{\partial \tilde{u}_{i}}{\partial \alpha_{i}^{t}}(\alpha) \geq \frac{\partial \tilde{u}_{i}}{\partial \alpha_{i}^{t^{\prime \prime}}}(\alpha)
$$

Observe that $v_{1, \alpha}^{t^{\prime \prime}}(i)=0$, while $v_{1, \alpha}^{t}(i)>0$.

We conclude that 11 is satisfied. Due to concavity of $\tilde{u}_{i}$, and hence concavity of the Lagrangian, the KKT conditions are necessary and sufficient for a global maximum of $L$. This concludes our proof.

\subsubsection{Uniqueness of Nash equilibrium}

Example 6.8 visualizes the computation of a Nash equilibrium. Furthermore it shows that the best response correspondence is not necessarily single valued and that uniqueness of a Nash equilibrium need not be satisfied.

Example 6.8. Consider the following dynamic $T U$-game $\Gamma=\left(v^{1}, v^{2}, u_{1}, u_{2}\right)$, the Shapley value and the first transfer system. The game $v^{1}$ is described by $v^{1}(1)=0, v^{1}(2)=$ $v^{1}(12)=1$. For $v^{2}$ we have $v^{2}(1)=v^{2}(2)=0, v^{2}(12)=1$. Let a $\in \mathbb{R}$. For $i \in\{1,2\}$ let $u_{i}\left(S h_{i}^{1}, S h_{i}^{2}\right)=a S h_{i}\left(v_{\alpha}^{1}\right)+a S h_{i}\left(v_{\alpha}^{2}\right)$. Notice that player one is a dummy player who can only make zero transfers. For player two we derive the following marginal utilities with respect to $\alpha_{2}^{1}$ and $\alpha_{2}^{2}$.

$$
\begin{aligned}
& \frac{\partial \tilde{u}_{2}}{\partial \alpha_{2}^{1}}=a S h_{2}\left(1_{2}\right) \\
& \frac{\partial \tilde{u}_{2}}{\partial \alpha_{2}^{2}}=a S h_{2}\left(1_{2}\right)
\end{aligned}
$$

Hence conditions 11 in Theorem 6.3 is satisfied for any $\alpha_{2} \in \mathcal{D}_{2}^{1}$. We conclude that all possible strategy combinations are Nash equilibria. This contradicts uniqueness of a Nash equilibrium.

Remark 6.2. If one considers a class of intertemporal utility functions where

$$
\frac{\partial u_{i}(\mathbf{x})}{\partial x^{t}} \geq \frac{\partial u_{i}(\mathbf{x})}{\partial x^{t^{\prime}}}\left[\frac{\partial u_{i}(\mathbf{x})}{\partial x^{t}}>\frac{\partial u_{i}(\mathbf{x})}{\partial x^{t^{\prime}}}\right]
$$


for all $t<t^{\prime}$ and $\Phi$ is linear then for all the players that are endowed with such an utility function it is always a best response to transfer as much as possible ${ }^{12}$ to the first period. This is true for any of the above transfer systems. ${ }^{13}$ If the utility function of agent $i$ is as above and $\Phi$ satisfies relative invariance under strategic equivalence and $\Phi(z)=0$ then in $G_{2}$ it is always a best response to transfer as much as possible to the first period. ${ }^{14}$

Observe that the discounted utility function $u(\mathbf{x})=\sum_{t=1}^{T} \delta^{t-1} x^{t}$ satisfies 17 with strict inequality. Under all transfer systems it is then a unique best response for each player to transfer as much as possible to period one. This leads to uniqueness of Nash equilibrium in $G_{1}$ as the feasible responses of a player are independent of what other players do.

However, in $G_{2}$ and $G_{3}$ multiple Nash equilibria might arise. The following example illustrates this:

Example 6.9. Let $N=\{1,2\}$ and $T=2$. Consider the sequence of $T U$-games $v^{t}(1)=$ $v^{t}(2)=1, v^{t}(12)=3$ for $t=1,2$. Let $\Phi$ be the Shapley value and let $u_{i}\left(\left(\operatorname{Sh}_{i}\left(v^{t}\right)\right)_{t=1}^{T}\right)$ be a discounted utility function as illustrated above with $\delta \in(0,1)$ for each agent. Consider the third transfer system. Observe that $c_{\{1,2\}}^{t}(1)+c_{\{1,2\}}^{t}(2)>v(12)$ for $t=1,2$. Suppose player 1 chooses transfers

$$
\alpha=\left(\left(\begin{array}{ccc}
1 & 0 & 2 \\
-1 & 0 & -2
\end{array}\right),\left(\begin{array}{lll}
0 & 0 & 0 \\
0 & 0 & 0
\end{array}\right)\right) \in S_{N}^{3} .
$$

It is then a unique best response of player 2 to play

$$
\alpha^{\prime}=\left(\left(\begin{array}{ccc}
1 & 0 & 2 \\
-1 & 0 & -2
\end{array}\right),\left(\begin{array}{ccc}
0 & 1 & 1 \\
0 & -1 & -1
\end{array}\right)\right) \in S_{N}^{3}
$$

as $\tilde{u}_{2}\left(\alpha^{\prime}\right)>\tilde{u}_{2}\left(\alpha^{\prime \prime}\right)$ for all $\alpha^{\prime \prime} \in \mathcal{D}_{2}^{3}(\alpha)$. Given player 2 plays $\alpha^{\prime}$ observe that $\alpha^{\prime} \in$ $B R_{1}^{3}\left(\alpha^{\prime}\right)$, hence $\alpha^{\prime}$ is a Nash equilibrium.

However, suppose player 1 plays

$$
\beta=\left(\left(\begin{array}{ccc}
1 & 0 & 1.5 \\
-1 & 0 & -1.5
\end{array}\right),\left(\begin{array}{lll}
0 & 0 & 0 \\
0 & 0 & 0
\end{array}\right)\right) \in S_{N}^{3} .
$$

\footnotetext{
${ }^{12}$ Recall that how much agents can transfer depends crucially on the transfer system considered.

${ }^{13}$ Berden (2007) provides a proof of this statement for transfer system one and two. However, when considering the second transfer system she assumes that each of the (resulting) cooperative games is weakly superadditive. One can then reformulate the above statement in terms of (weakly) dominant strategies.

${ }^{14} \mathrm{~A}$ proof is provided in Berden (2007) for the case that the cooperative games are weakly superadditive.
} 
It is then a unique best response of player 2 to play

$$
\beta^{\prime}=\left(\left(\begin{array}{ccc}
1 & 0 & 1.5 \\
-1 & 0 & -1.5
\end{array}\right),\left(\begin{array}{ccc}
0 & 1 & 1.5 \\
0 & -1 & -1.5
\end{array}\right)\right) \in S_{N}^{3}
$$

as $\tilde{u}_{2}\left(\beta^{\prime}\right)>\tilde{u}_{2}\left(\beta^{\prime \prime}\right)$ for all $\beta^{\prime \prime} \in \mathcal{D}_{2}^{3}(\alpha)$. Given player 2 plays $\beta^{\prime}$ we have $\beta^{\prime} \in B R_{1}^{3}\left(\beta^{\prime}\right)$ and hence $\beta^{\prime}$ is a Nash equilibrium. We conclude that a Nash equilibrium need not be unique when agents have discounted utility functions.

\subsection{TRANSFER OF INITIAL ENDOWMENTS}

In the previous section we discussed individuals' transfers of worth from some periods to others. Although the third transfer system is quite general and can be applied to many different classes of TU-games, for some TU-games there are other transfer systems that seem more natural. In this section we want to focus on transfers of initial endowments in market games (Shapley \& Shubik, 1969). A market is a tuple $(N, P, A, F)$, where $N$ is a finite set of traders, $P$ is the set of $m$ continuous goods, $A=\left(a_{i}\right)_{i \in N}$ with $a_{i} \in \mathbb{R}_{+}^{m}$ is the vector of initial endowments, $F=\left(f_{i}\right)_{i \in N}$ is a production function vector. We assume that for each $i \in N, f_{i}$ is continuous and concave. A market game can be generated from the underlying market in the following way:

$$
v(S)=\max \left\{\sum_{i \in S} f_{i}\left(x_{i}\right) \mid x_{i} \in \mathbb{R}_{+}^{m}, \sum_{i \in S} x_{i}=\sum_{i \in S} a_{i}\right\}
$$

for all $S \subseteq N$. We define the set of all market games with player set $N$ by $\mathcal{M}^{N}$.

We consider a finite sequence of market games, and assume that each agent is endowed with an intertemporal utility function $u_{i}$ as defined in Section 6.3.

Definition 6.11. A dynamic market game $\Lambda$ is a pair $(\mathbf{v}, \mathbf{u})$ where

$$
\begin{aligned}
& \text { 1. } \mathbf{v}=\left(v^{1}, \ldots, v^{T}\right) \in\left(\mathcal{M}^{N}\right)^{T} \\
& \text { 2. } \mathbf{u}=\left(u_{1}, \ldots, u_{n}\right)
\end{aligned}
$$

As in Section 6.3, we apply a solution concept to the dynamic market game. Let us first clarify that all of the above values are defined for market games. Let $v \in \mathcal{M}^{N}$. As all values except the $\tau$-value and the nucleolus are defined for all classes of TU-games we only have to argue that $\tau(v) \neq \emptyset$ and $\nu(v) \neq \emptyset$. Note that each market game is balanced (Shapley \& Shubik, 1969) and hence the core of the game is nonempty, by the Bondareva-Shapley Theorem (Bondareva, 1963; Shapley, 1967). If the core of a game is nonempty, then the nucleolus lies in the core (and hence is well defined). Moreover, the 
$\tau$-value is defined for quasi-balanced games. The class of balanced games constitutes a subclass of the class of quasi-balanced games (Tijs \& Lipperts, 1982) and hence, the $\tau$-value is well defined.

We assume that individuals can transfer the sum of their initial endowments among periods and thereby affect their intertemporal payoffs.

\subsubsection{Strategic interaction}

Definition 6.12. With each $\Lambda=(\mathbf{v}, \mathbf{u})$ and a value $\Phi$ we associate the non-cooperative game $G(\Lambda, \Phi)=\left(\mathcal{S}_{1}, \ldots, \mathcal{S}_{n} ; \tilde{u}_{1}, \ldots, \tilde{u}_{n}\right)$. For all $i \in N$ let the

- strategy set $\mathcal{S}_{i}$ be the collection of vectors $\alpha_{i} \in \mathbb{R}^{T \times m}$ such that

1. $a_{i}^{t}+\alpha_{i}^{t} \geq \mathbf{0}$ for all $t \in\{1, \ldots, T\}$

2. $\sum_{t=1}^{T} \alpha_{i}^{t}=\mathbf{0}$.

- utility function $\tilde{u}_{i}: \mathcal{S}_{N} \rightarrow \mathbb{R}$ such that

$$
\tilde{u}_{i}\left(\alpha_{1}, \ldots, \alpha_{n}\right)=u_{i}\left(\Phi_{i}\left(\left(v_{\alpha}^{t}\right)_{t=1}^{T}\right)\right)
$$

where $v_{\alpha}^{t}$ is generated as follows:

$$
v_{\alpha}^{t}(S)=\max \left\{\sum_{i \in S} f_{i}^{t}\left(x_{i}\right) \mid x_{i} \in \mathbb{R}_{+}^{m}, \sum_{i \in S} x_{i}=\sum_{i \in S} a_{i}^{t}+\sum_{i \in S} \alpha_{i}^{t}\right\}
$$

for all $S \subseteq N$.

The conditions on players' strategies are in the same spirit as the ones in Section 6.3. The first condition ensures that agents cannot remove more than their initial endowment from a period. The second condition reflects the idea that making transfers is free of charge but also by making transfers no additional initial endowments can be generated. Note that in this set up, there are no constraints across players' strategies. Observe that the strategy set of players' is never empty, as they can always make zero transfers.

As in the previous section we have to guarantee that $\tilde{u}_{i}(\alpha)$ and $\Phi_{i}\left(v_{\alpha}\right)$ are well defined for all $i \in N$, all $\alpha \in \mathcal{S}_{i}$ and all $t \in\{1, \ldots, T\}$. We have already argued that (at least) the solution concepts discussed above are well defined for market games. The next example illustrates that $\left(\Phi_{i}\left(v_{\alpha}^{t}\right)\right)_{t=1}^{T}$ need not be in the domain of $u_{i}$.

Example 6.10. Let $N=\{1,2\}$ and $T=2$. There is a single production factor and $a_{i}=(1,1)$ for both players. Further assume that both players produce with the linear production function $f_{i}^{t}\left(x_{i}\right)=x_{i}$ in each period. Let $\Phi$ be the Shapley value. Let the 
intertemporal utility function of both players be $u_{i}\left(S h_{i}\left(v_{\alpha}^{1}\right), S h_{i}\left(v_{\alpha}^{2}\right)\right)=\ln \left(S h_{i}\left(v_{\alpha}^{1}\right)\right)+$ $\ln \left(S h_{i}\left(v_{\alpha}^{2}\right)\right)$. The derived market game is as follows: $v^{t}(1)=v^{t}(2)=1, v^{t}(12)=2$ for $t=1,2$. Suppose both agents make transfers $\alpha_{i}=(-1,1)$ which means that they shift their total initial endowments to period 2. The dynamic market game after transfers is then $v_{\alpha}^{1}=z$ and $v_{\alpha}^{2}(1)=v_{\alpha}^{2}(2)=2, v_{\alpha}^{2}(12)=4$. Note that $S h_{i}(z)=0$ for all players. Hence, $\left(\Phi_{i}\left(v_{\alpha}^{t}\right)\right)_{t=1}^{T}$ is not in the domain of $u_{i}$.

We make the same approach as in the previous section and define feasible tuple.

Definition 6.13. Fix a value $\Phi$ for $\mathcal{M}^{N}$. A tuple $(\mathbf{u}, \mathbf{v})$ is feasible for $G(\Lambda, \Phi)$, if for all $i \in N$, all $\alpha \in \mathcal{S}_{N}$ and $t \in\{1, \ldots, T\}$

1. $\Phi$ is well defined for all $v_{\alpha}^{t}$ and

2. $\left(\Phi_{i}\left(v_{\alpha}^{t}\right)\right)_{t=1}^{T}$ is in the domain of $u_{i}$.

The above definition ensures that $\tilde{u}_{i}$ is well defined for all $i \in N$ and $\alpha \in \mathcal{S}_{N}$ given $\Phi$. In the sequel of the chapter all results are with respect to feasible tuples for the corresponding non-cooperative game.

The following example shows that transfers of initial endowments cannot be fully captured by the third transfer system of the previous section.

Example 6.11. Let $N=\{1,2\}$ and $T=2$. There is one production factor and $f_{i}^{1}\left(x_{i}\right)=$ $\sqrt{x_{i}}$ and $f_{i}^{2}\left(x_{i}\right)=x_{i}$ for each $i \in N$. Moreover, assume that $a_{i}=(4,5)$ for each player. The resulting games are $v^{1}(1)=v^{1}(2)=2, v^{1}(12)=4$ and $v^{2}(1)=v^{2}(2)=$ $5, v^{2}(12)=10$. Suppose agent one transfers $\alpha_{1}=(5,-5)$ and player 2 doesn't make any transfer. The new games are $v_{\alpha}^{1}(1)=3, v_{\alpha}^{1}(2)=2, v^{1}(12)=\sqrt{26}$ and $v_{\alpha}^{2}(1)=$ $0, v_{\alpha}^{2}(2)=v_{\alpha}^{2}(12)=5$. It is impossible to model this changes in the underlying games using transfers as specified under the third transfer system. This is due to the different production functions of the players in the two periods.

This justifies further investigation of the non-cooperative games $G(\Lambda, \Phi)$.

\subsubsection{Existence of Nash equilibria}

One of our main goals is to prove existence of Nash equilibria in the above game. We say a strategy $\alpha_{i} \in \mathcal{S}_{i}$ is a best response of player $i$ to $\alpha_{-i} \in \mathcal{S}_{-i}$ if $\tilde{u}_{i}\left(\alpha_{i}, \alpha_{-i}\right) \geq \tilde{u}_{i}\left(\alpha_{i}^{\prime}, \alpha_{-i}\right)$ for all $\alpha_{i}^{\prime} \in \mathcal{S}_{i}$. We denote the set of best responses of player $i$ to profile $\alpha_{-i}$ by $B_{i}\left(\alpha_{-i}\right)$. In order to prove existence of a Nash equilibrium we have to show that there is $\alpha \in \mathcal{S}_{N}$ such that $\alpha \in B(\alpha)$, i.e. $B$ has a fixed point. We make use of Kakutani's fixpoint theorem (Kakutani, 1941). 
Theorem 6.4 (Kakutani (1941)). Let $X$ be a non-empty, closed, bounded, and convex subset of $\mathbb{R}^{n}$. If $f: X \rightrightarrows X$ is convex-valued and has a closed graph then $f$ has a fixed point, i.e., there is $x \in X$ such that $x \in f(x)$.

The following Theorem states sufficient conditions for a Nash equilibrium in $G(\Lambda, \Phi)$ to exist.

Theorem 6.5. Let $\Lambda=(\mathbf{u}, \mathbf{v})$ be a dynamic market game where in each period all agents have a concave and continuous production function. Let $\Phi$ be increasing and concave and $u_{i}$ be increasing, quasiconcave and continuous for all $i \in N$. Then a Nash equilibrium exists in $G=(\Lambda, \Phi)$.

Proof. We first show that $\mathcal{S}_{N}$ is a closed, bounded and convex subset of the Euclidean space. By definition $\mathcal{S}_{N}=\times_{i \in N} \mathcal{S}_{i}$. Since $\mathcal{S}_{i}$ is a simplex, we have that $\mathcal{S}_{i}$ is closed and bounded. It is easy to see that for any $\alpha_{i}^{\prime}$ and $\alpha_{i}^{\prime \prime} \in \mathcal{S}_{i}$ and $\lambda \in(0,1)$ we have that $\lambda \alpha_{i}^{\prime}+(1-\lambda) \alpha_{i}^{\prime \prime} \in \mathcal{S}_{i}$. Hence $\mathcal{S}_{i}$ is convex. The product set of compact convex sets is compact and convex, hence $\mathcal{S}_{N}$ is compact and convex.

Secondly, we have to show that $B(\alpha)$ is non-empty for every $\alpha \in \mathcal{S}_{N}$. Since $f^{t}$ is continuous for all $t \in\{1, \ldots, T\}, u_{i}$ are continuous for all $i \in N$ and $\Phi$ is continuous, we have that $\tilde{u}_{i}$ is continuous for every $i \in N$. By definition

$$
B_{i}\left(\alpha_{-i}\right)=\operatorname{argmax}_{\alpha_{i} \in \mathcal{S}_{i}} \tilde{u}_{i}\left(\alpha_{i}, \alpha_{-i}\right),
$$

where $\mathcal{S}_{i}$ is non-empty and compact. By Weierstrass Extreme Value Theorem we have that $B(\alpha)$ is non-empty.

Further, we show that $B$ is a convex-valued correspondence. Fix any arbitrary $\beta, \gamma \in$ $B_{i}\left(\alpha_{-i}\right)$. Then for all $\lambda \in(0,1)$ we have:

$$
\begin{aligned}
& \tilde{u}_{i}\left(\beta, \alpha_{-i}\right) \geq \tilde{u}_{i}\left(\alpha_{i}, \alpha_{-i}\right) \text { for all } \alpha_{i} \in \mathcal{S}_{i} \\
& \tilde{u}_{i}\left(\gamma, \alpha_{-i}\right) \geq \tilde{u}_{i}\left(\alpha_{i}, \alpha_{-i}\right) \text { for all } \alpha_{i} \in \mathcal{S}_{i} .
\end{aligned}
$$

In a first step we are going to show that $v_{\left(\lambda \beta+(1-\lambda) \gamma, \alpha_{-i}\right)}^{t}(S) \geq \lambda v_{\left(\beta, \alpha_{-i}\right)}^{t}(S)+(1-$ $\lambda) v_{\left(\gamma, \alpha_{-i}\right)}^{t}(S)$ for all $S \subseteq N$ and $t \in\{1, \ldots, T\}$.

Let $t \in\{1, \ldots, T\}$ and $S \subseteq N$. Moreover, let $x^{*}$ be the maximizer of $\sum_{i \in S} f_{i}^{t}\left(x_{i}\right)$ given the constraint $\sum_{i \in S} x_{i}=\sum_{i \in S} a_{i}^{t}+\sum_{j \in S \backslash\{i\}} \alpha_{j}^{t}+\beta^{t}$ and $x^{* *}$ be the maximizer 
given the constraint $\sum_{i \in S} x_{i}=\sum_{i \in S} a_{i}^{t}+\sum_{j \in S \backslash\{i\}} \alpha_{j}^{t}+\gamma^{t}$, respectively. We show that $\lambda x^{*}+(1-\lambda) x^{* *}$ is feasible under $\sum_{i \in S} x_{i}=\sum_{i \in S} a_{i}^{t}+\sum_{j \in S \backslash\{i\}} \alpha_{j}^{t}+\lambda \beta^{t}+(1-\lambda) \gamma^{t}$.

$$
\begin{aligned}
& \sum_{i \in S}\left(\lambda x_{i}^{*}+(1-\lambda) x_{i}^{* *}\right) \\
& =\lambda \sum_{i \in S} x_{i}^{*}+(1-\lambda) \sum_{i \in S} x_{i}^{* *} \\
& =\lambda\left(\sum_{i \in S} a_{i}^{t}+\sum_{j \in S \backslash\{i\}} \alpha_{j}^{t}+\beta^{t}\right)+(1-\lambda)\left(\sum_{i \in S} a_{i}^{t}+\sum_{j \in S \backslash\{i\}} \alpha_{j}^{t}+\gamma^{t}\right) \\
& =\sum_{i \in S} a_{i}^{t}+\sum_{j \in S \backslash\{i\}} \alpha_{j}^{t}+\lambda \beta^{t}+(1-\lambda) \gamma^{t}
\end{aligned}
$$

Observe that this is true for all $t \in\{1, \ldots, T\}$ and all $S \subseteq N$. As $f_{i}^{t}$ is concave for all $i \in N$ and $t=1, \ldots, T$, we have that $\sum_{i \in S} f_{i}^{t}$ is a concave function, as well. Due to feasibility of $\lambda x^{*}+(1-\lambda) x^{* *}$ and concavity of $\sum_{i \in S} f_{i}^{t}$ it follows that $v_{\left(\lambda \beta+(1-\lambda) \gamma, \alpha_{-i}\right)}^{t}(S) \geq$ $\lambda v_{\left(\beta, \alpha_{-i}\right)}^{t}(S)+(1-\lambda) v_{\left(\gamma, \alpha_{-i}\right)}^{t}(S)$ for all $S \subseteq N$ and $t \in\{1, \ldots, T\}$.

We now show that $\tilde{u}_{i}\left(\lambda \beta+(1-\lambda) \gamma, \alpha_{-i}\right) \geq \tilde{u}_{i}\left(\alpha_{i}, \alpha_{-i}\right)$ for all $\alpha_{i} \in \mathcal{S}_{i}$.

$$
\begin{aligned}
& \tilde{u}_{i}\left(\lambda \beta+(1-\lambda) \gamma, \alpha_{-i}\right) \\
& =u_{i}\left(\Phi_{i}\left(v_{\left(\lambda \beta+(1-\lambda) \gamma, \alpha_{-i}\right)}^{t}\right)_{t=1}^{T}\right) \\
& \geq u_{i}\left(\Phi_{i}\left(\lambda v_{\left(\beta, \alpha_{-i}\right)}^{t}+(1-\lambda) v_{\left(\gamma, \alpha_{-i}\right)}^{t}\right)_{t=1}^{T}\right) \\
& \geq u_{i}\left(\lambda \Phi_{i}\left(v_{\left(\beta, \alpha_{-i}\right)}^{t}\right)_{t=1}^{T}+(1-\lambda) \Phi_{i}\left(v_{\left(\gamma, \alpha_{-i}\right)}^{t}\right)_{t=1}^{T}\right) \\
& \geq \min \left\{u_{i}\left(\Phi_{i}\left(v_{\left(\beta, \alpha_{-i}\right)}^{t}\right)_{t=1}^{T}\right), u_{i}\left(\Phi_{i}\left(v_{\left(\gamma, \alpha_{-i}\right)}^{t}\right)_{t=1}^{T}\right)\right\} \\
& =\min \left\{\tilde{u}_{i}\left(\beta, \alpha_{-i}\right), \tilde{u}_{i}\left(\gamma, \alpha_{-i}\right)\right\}
\end{aligned}
$$

Where the first equality follows from the definition of $\tilde{u}_{i}$. The first inequality follows from the fact that $u_{i}$ and $\Phi_{i}$ are increasing, together with $v_{\left(\lambda \beta+(1-\lambda) \gamma_{\left., \alpha_{-i}\right)}\right.}^{t}(S) \geq$ $\lambda v_{\left(\beta, \alpha_{-i}\right)}^{t}(S)+(1-\lambda) v_{\left(\gamma, \alpha_{-i}\right)}^{t}(S)$ for all $S \subseteq N$ and $t \in\{1, \ldots, T\}$. The second inequality follows from concavity of $\Phi$. The third inequality follows from quasiconcavity of $u_{i}$. The last equality follows from the definition of $\tilde{u}_{i}$. Hence we conclude that $\tilde{u}_{i}\left(\lambda \beta+(1-\lambda) \gamma, \alpha_{-i}\right) \geq \tilde{u}_{i}\left(\alpha_{i}, \alpha_{-i}\right)$ for all $i \in N$. Thus $B_{i}\left(\alpha_{-i}\right)$ is a convex set. Since $B(\alpha)$ is then a product set over convex sets, we conclude that $B(\alpha)$ is a convex set for all $\alpha \in \mathcal{S}_{N}$.

It remains to show that $B(\alpha)$ has a closed graph. Let $\left(\alpha_{i}^{n}, \alpha_{-i}^{n}\right) \rightarrow\left(\alpha_{i}, \alpha_{-i}\right)$ such that $\alpha_{i}^{n} \in B_{i}\left(\alpha_{-i}^{n}\right)$ for all $n$. Then for all $n \in \mathbb{N}$ we have, $\tilde{u}_{i}\left(\alpha_{i}^{n}, \alpha_{-i}^{n}\right) \geq \tilde{u}_{i}\left(\alpha_{i}^{\prime}, \alpha_{-i}^{n}\right)$ for all 
$\alpha_{i}^{\prime} \in \mathcal{S}_{i}$. By continuity of $\tilde{u}_{i}$ it follows that $\tilde{u}_{i}\left(\alpha_{i}, \alpha_{-i}\right) \geq \tilde{u}_{i}\left(\alpha_{i}^{\prime}, \alpha_{-i}\right)$. This implies that $\alpha_{i} \in B_{i}\left(\alpha_{-i}\right)$.

Recall that all linear solutions mentioned in Section 6.3 satisfy concavity.

\subsubsection{Uniqueness of Nash equilibria}

In this section we elaborate on uniqueness of Nash equilibria. The first example illustrates, that Nash equilibria need not be unique.

Example 6.12. Let $N=\{1,2\}$, and $T=2$. There is a single production factor. Moreover, $a_{i}=(1,1)$ for $i=1,2$ and suppose that all agents produce in each period with production function $f_{i}^{t}\left(x_{i}\right)=x_{i}$. Moreover let $u_{i}\left(\left(\Phi_{i}\left(v_{\alpha}^{t}\right)\right)_{t=1}^{T}\right)=\Phi_{i}^{1}\left(v_{\alpha}^{1}\right)+\Phi_{i}^{2}\left(v_{\alpha}^{2}\right)$ for $i=1,2$. Let $\Phi_{i}$ be the Shapley value. The resulting dynamic market game is $v^{t}(1)=1, v^{t}(2)=$ $1, v^{t}(12)=2$ for $t=1,2$. Also, Sh $\left(v^{t}\right)=(1,1)$ for $t=1,2$. Because of symmetry of the game in all it's components, $\tilde{u}_{i}\left(\alpha_{1}, \alpha_{2}\right)=2$ for all $\alpha_{1} \in \mathcal{S}_{1}$ and $\alpha_{2} \in \mathcal{S}_{2}$. Thus, any strategy combination $\left(\alpha_{1}, \alpha_{2}\right) \in \mathcal{S}_{N}$ constitutes a Nash equilibrium.

Obviously, there are games $G(\lambda, \Phi)$ in which the Nash equilibrium is unique.

Example 6.13. Let $N=\{1,2\}$ and $T=2$. There is a single production factor. Moreover, let $a_{1}=(2,0), a_{2}=(0,2)$, and suppose $f_{i}^{1}\left(x_{i}\right)=x_{i}$ for $i=1,2$ and $f_{i}^{2}\left(x_{i}\right)=\frac{1}{2} x_{i}$ for $i=1,2$. Further, let $u_{i}\left(\left(\Phi_{i}\left(v_{\alpha}^{t}\right)\right)_{t=1}^{2}\right)=\Phi_{i}\left(v_{\alpha}^{1}\right)+\frac{1}{2} \Phi_{i}\left(v_{\alpha}^{2}\right)$ for $i=1,2$. Let $\Phi$ be the Shapley value. The resulting dynamic market game is then $v^{1}(1)=2, v^{1}(2)=$ $0, v^{1}(12)=2$ and $v^{2}(1)=0, v^{2}(2)=1, v^{2}(12)=1$. Also, $\operatorname{Sh}\left(v^{1}\right)=(2,0), \operatorname{Sh}\left(v^{2}\right)=$ $(0,1)$. Consider $G(\Lambda, S h)$. Independent of the strategy of player 2 , it is a dominant strategy for player 1 to not make any transfer, hence playing $\alpha_{1}=(0,0)$, as $\tilde{u}_{1}\left((0,0), \alpha_{2}\right)=2>\tilde{u}_{1}\left(\alpha_{1}^{\prime}, \alpha_{2}\right)$ for all $\alpha_{1}^{\prime} \neq(0,0)$ and $\alpha_{2} \in \mathcal{S}_{2}$. Analogously, it is a dominant strategy for player 2 to play $\alpha_{2}=(2,-2)$, and, hence, transferring all her initial endowment to period 1. A combination of dominant strategies is always a Nash equilibrium and moreover it is unique.

In section 6.3.5 we showed that under the first transfer system Nash equilibria are unique when agents are endowed with discounted utility functions. Example 6.14 illustrates that in this set up, even when agents are endowed with discounted utility functions, it need not be a best response to transfer as much as possible to the first period.

Example 6.14. Let $N=\{1,2\}$ and $T=2$. There is a single production factor and each agent is endowed with a discounted utility function as specified above with discount factor $\delta=\frac{1}{2}$. Each agent produces with production functions $f_{i}^{1}\left(x_{i}\right)=\sqrt{x_{i}}$ and $f_{i}^{2}\left(x_{i}\right)=x_{i}$. Moreover, assume that $a_{i}=(4,5)$ for each player. Let $\Phi$ be the Shapley value. The 
resulting games are $v^{1}(1)=v^{1}(2)=2, v^{1}(12)=4$ and $v^{2}(1)=v^{2}(2)=5, v^{2}(12)=10$. Observe that $u_{1}\left(S h_{1}\left(v^{1}\right), S h_{1}\left(v^{2}\right)\right)=2+5 \cdot \frac{1}{2}=4 \frac{1}{2}$. Suppose agent one transfers $\alpha_{1}=$ $(5,-5)$ and player 2 doesn't make any transfer. The new games are $v_{\alpha}^{1}(1)=3, v_{\alpha}^{1}(2)=$ $2, v^{1}(12)=\sqrt{26}$ and $v_{\alpha}^{2}(1)=0, v_{\alpha}^{2}(2)=v_{\alpha}^{2}(12)=5$. The Shapley value for player one is $S h_{1}\left(v_{\alpha}^{1}\right) \approx 3 \frac{1}{20}$ and $\left.S h_{1}\left(v_{\alpha}^{2}\right)\right)=0$, hence $u_{1}\left(\left(S h_{1}\left(v^{1}\right), S h_{1}\left(v^{2}\right)\right) \approx 3 \frac{1}{20}<4 \frac{1}{2}\right.$. We conclude that it is not a (weakly) dominant strategy for player 1 to transfer all her initial endowment to period 1.

\subsection{TRANSFER OF POWER}

The case of market games showed that for some classes of TU-games different transfer systems seem in order. This fact immediately brings the motivation to investigate a different setting, for instance voting games. This is what we aim to do in this section. Casella (2005) introduces the idea of storable votes. The paper is motivated by the need for more flexible decision-making mechanisms in the European Union. A voting scheme for binary decisions taken by committees that meet regularly over time is proposed. At each meeting, committee members are allowed to store their vote for future use. Although the model in Casella (2005) is different from the approach we follow, the main idea remains the same. The possibility of shifting votes intertemporally allows agents to concentrate their votes when preferences are more intense or if they cannot participate in all meetings due to time constraints. A simple game is a mapping $v: 2^{N} \mapsto\{0,1\}$ such that $v(\emptyset)=0, v(N)=1$ and $v(S) \leq v(T)$ for all $S, T \in 2^{N}$ with $S \subset T$. A coalition $S \in 2^{N}$ is winning if $v(S)=1$ and losing if $v(S)=0$. We call $S \subseteq N$ a minimal winning coalition if every proper subset of $S$ is a losing coalition. The set of minimal winning coalitions is defined as

$$
M=\{S \subseteq N \mid v(S)=1 \text { and for all } T \subset S \text { we have } v(T)=0\}
$$

Voting games are simple games which reflect the distribution of voting power within, for instance, political systems. A weight vector $\mathbf{w}=\left(w_{1}, \ldots, w_{n}\right) \in \mathbb{R}^{N}$ together with a real number $q, 0<q \leq \sum_{i \in N} w_{i}$, induces a simple game $v$ via

$$
v(S)= \begin{cases}1 & \text { if } \sum_{i \in S} w_{i} \geq q \\ 0 & \text { otherwise }\end{cases}
$$

We call $v$ a weighted voting game if a representation by some $(\mathbf{w}, q)$ exists. In this case, $w_{i}$ is the voting strength or weight of player $i \in N$ and $q$ is the majority level or quota: once a coalition accumulates sufficient total voting strength in order to exceed 
the quota $q$ it is winning. We consider proper weighted voting games. A voting game is proper if $v(S)=1$ implies $v(N \backslash S)=0$ for every $S \subseteq N$. Hence, we shall assume that $q>\frac{1}{2} \sum_{i \in N} w_{i}$. We denote by $\mathcal{V}$ the set of all proper weighted voting games. A power index $P: \mathcal{V} \cup\{z\} \mapsto \mathbb{R}^{N}$ is a value applied to weighted voting games and the zero game such that $P_{i}(z)=0$ for all $i \in N$. We extend the domain of a power index by including the zero game, as it might be the case that all agents transfer all their weights away from one period and hence the resulting game will not be a proper weighted voting game. ${ }^{15}$ The payoff vector assigned to a game is interpreted as reflecting power distribution.

\section{Shapley-Shubik power index}

This index was introduced in Shapley \& Shubik (1954) and is based on the Shapley value discussed above. The idea is that a group of individuals votes in order one after another. The first voter, that is by joining making a coalition winning is given the credit. If one chooses the order of voters randomly, one can compute how often a given individual is pivotal.

We say $i$ is swing for coalition $S$ if $v(S)=1$ and $v(S \backslash\{i\})=0$. The ShapleyShubik index is then defined as:

$$
\phi_{i}(v):=\sum_{i \text { is swing for } S} \frac{(|S|-1) !(|N|-|S|) !}{|N| !}
$$

\section{Banzhaf power index}

The Banzhaf power index was introduced in Banzhaf (1965). The idea is that the power of voter $i$ should be proportional to the number of coalitions for which $i$ is a swing voter. Let $\eta_{i}(v)$ denote the number of swings of individual $i$ and let $\bar{\eta}(v)=\sum_{i \in N} \eta_{i}(v)$ denote the total number of swings in $v$.

The non-normalized Banzhaf index expresses the swing probabilities of each player and is defined as:

$$
\beta_{i}(v):=\frac{\eta_{i}(v)}{2^{|N|-1}} .
$$

We can also consider the normalized Banzhaf index, that is defined as:

$$
\beta_{i}^{\prime}(v):=\frac{\eta_{i}(v)}{\bar{\eta}(v)}
$$

Note that $\sum_{i \in N} \beta_{i}^{\prime}(v)=1$.

\section{Deegan-Packel power index}

Introduced in Deegan \& Packel (1978) it is based on the following assumptions:

\footnotetext{
${ }^{15}$ However, intuitively this situation will not occur in equilibrium, as each player can make herself winning by transferring a marginal amount of weight to this period.
} 
1. Only minimal winning coalitions will form.

2. Each minimal winning coalition has an equal probability of forming.

3. Players in minimal winning coalition divide the power equally.

Hence,

$$
\rho_{i}(v):=\frac{1}{|M|} \sum_{S \in M_{i}} \frac{1}{|S|} .
$$

We consider a finite sequence of weighted voting games. Again, we assume that individuals are endowed with an intertemporal utility function $u_{i}: \mathbb{R}^{\mathbb{T}} \mapsto \mathbb{R}$.

Definition 6.14. A dynamic weighted voting game $\Sigma$ is a pair $(\mathbf{u}, \mathbf{v})$ where:

1. $\mathbf{v}=\left(v^{1}, \ldots, v^{T}\right) \in(\mathcal{V} \cup\{z\})^{T}$ and

2. $\mathbf{u}=\left(u_{1}, \ldots, u_{n}\right)$.

A power index $P$ is applied to a dynamic voting game by applying it to each period separately. We assume that individuals can transfer power in form of their weights among periods.

Again, let $v_{\alpha}^{t}$ be the resulting voting game in period $t$ after transfers $\alpha$.

Definition 6.15. With each $\Sigma$ and value $P$ we associate the non-cooperative game $G(\Sigma, P)=\left(\mathcal{S}_{1}, \ldots, \mathcal{S}_{n} ; \tilde{u}_{1}, \ldots, \tilde{u}_{n}\right)$. For all $i \in N$ let the

- strategy set $\mathcal{S}_{i}$ be the collection of vectors $\alpha_{i} \in \mathbb{R}^{T}$ such that

1. $w_{i}^{t}+\alpha_{i}^{t} \geq 0$ for all $t \in\{1, \ldots, T\}$

2. $\sum_{t=1}^{T} \alpha_{i}^{t}=0$.

- utility function $\tilde{u}_{i}: \mathcal{S}_{N} \rightarrow \mathbb{R}$ such that

$$
\tilde{u}_{i}\left(\alpha_{1}, \ldots, \alpha_{n}\right)=u_{i}\left(P_{i}\left(\left(v_{\alpha}^{t}\right)_{t=1}^{T}\right)\right) .
$$

Note that this non-cooperative game is not a special case of any of the non-cooperative games considered in the previous sections. We first provide an example that a Nash equilibrium need not exist in the above game.

Example 6.15. Let $N=\{1,2\}$ and $w_{1}^{1}=w_{1}^{2}=\frac{1}{3}, w_{2}^{1}=w_{2}^{2}=\frac{2}{3}$ and let $S \subseteq N$ be winning, if $\sum_{i \in S} w_{i}>\frac{\sum_{i \in N} w_{i}}{2}$. The resulting dynamic voting game $\sum$ is $v^{t}(1)=0, v^{t}(2)=$ $1, v^{t}(12)=1$ for $t=1,2$. The solution concept applied to each game is the normalized Banzhaf index $\beta^{\prime}$ and the intertemporal utility functions be $u_{i}\left(\beta_{i}^{\prime}\left(v^{1}\right), \beta_{i}^{\prime}\left(v^{2}\right)\right)=$ 
$\beta_{i}^{\prime}\left(v^{1}\right)+\beta_{i}^{\prime}\left(v^{2}\right)$ for $i=1,2$. Consider the non-cooperative $G\left(\Sigma, \beta^{\prime}\right)$ as defined above. First observe, that player two would never make a transfer that would make him losing in any of the periods. Observe that player one could make player two losing and hence gaining herself some payoff by transferring all her weight in one period. W.l.o.g. suppose player one is transferring all her weights to period one, hence playing $\alpha_{1}=\left(\frac{1}{3},-\frac{1}{3}\right)$. The resulting dynamic voting game is then $v_{\alpha}^{1}(1)=0, v^{1}(2)=0, v_{\alpha}^{1}(12)=1$ and $v_{\alpha}^{2}(1)=0, v_{\alpha}^{2}(2)=1, v^{2}(12)=1$, resulting in $\beta^{\prime}\left(v_{\alpha}^{1}\right)=\left(\frac{1}{2}, \frac{1}{2}\right)$ and $\beta^{\prime}\left(v_{\alpha}^{2}\right)=(0,1)$. But then, player two would deviate from $\alpha_{2}=(0,0)$ to $\alpha_{2}^{\prime}=(\epsilon,-\epsilon)$ with $\epsilon>0$. The weights are then

$$
w_{1}^{1}=\frac{2}{3}, w_{1}^{2}=0 \text { and } w_{2}^{1}=\frac{2}{3}+\epsilon, w_{2}^{2}=\frac{2}{3}-\epsilon .
$$

Applying the normalized Banzhaf index to the resulting dynamic voting game, we have $\beta^{\prime}\left(v_{\left(\alpha_{1}, \alpha_{2}^{\prime}\right)}^{t}\right)=(0,1)$ for $t=1,2$. Now, player one would deviate from her strategy by playing $\alpha_{1}^{\prime}=\left(-\frac{2}{3}, \frac{2}{3}\right)$, resulting in the weights $w_{1}^{1}=0, w_{1}^{2}=\frac{2}{3}$ and $w_{2}^{1}=\frac{2}{3}+$ $\epsilon, w_{2}^{2}=\frac{2}{3}-\epsilon$ and hence making her winning in the second period. Then $\beta^{\prime}\left(v_{\alpha^{\prime}}^{1}\right)=(0,1)$ and $\beta^{\prime}\left(v_{\alpha^{\prime}}^{2}\right)=(0,1)$. Now player two has incentive to deviate from her strategy, by playing $\alpha_{2}^{\prime \prime}=(-2 \epsilon, 2 \epsilon)$. Hence, two becomes winning in both periods, resulting in $\beta^{\prime}\left(v_{\left(\alpha_{1}^{\prime}, \alpha_{2}^{\prime \prime}\right)}^{t}\right)=(0,1)$ for $t=1,2$. This situation is symmetric to 19. Hence again player one would have an incentive to deviate from her strategy and so on. We result in a cycle and hence conclude, that no Nash equilibrium exists in this game.

Observe, that above example is also valid when applying the Shapley-Shubik, the Banzhaf and the Deegan-Packel power index.

The following example shows that Nash equilibria exist in some games and also demonstrates that Nash equilibria need not be unique. This justifies further research.

Example 6.16. Let $N=\{1,2,3\}, w_{i}^{t}=\frac{1}{3}$ for all $i \in N$ and $t=1, \ldots, T$ and let $S \subseteq N$ be winning, if $\sum_{i \in S} w_{i}>\frac{\sum_{i \in N} w_{i}}{2}$. The resulting voting game is $v^{t}(i)=0$ and $v^{t}(S)=1$ for all $S \subseteq N$ such that $|S| \geq 2$ and all $t=1,2,3$. The solution applied to each stage game is the Shapley-Shubik power index and $u_{i}\left(\phi_{i}\left(v_{\alpha}^{t}\right)_{t=1}^{3}\right)=\sum_{t=1}^{3} \phi_{i}\left(v_{\alpha}^{t}\right)$. It is easy to see that in equilibrium every player gets a payoff of 1 . Suppose this were not the case, then there is at least one player that has a payoff larger than one and, hence, there is at least one player that has a payoff smaller than 1, but then by making transfers of her voting power she can increase her payoff to one. Hence, we cannot have an equilibrium. We deduce that in this game several equilibria arise, for instance: all players make zero transfers, one player transfers all her voting power to the first period, another player transfers everything to the second and the third player everything to the third period.

It is left to future research to identify sufficient conditions for the existence of Nash equilibria in games where players can shift their voting weights over time. 


\subsection{FURTHER RESEARCH}

We elaborated on different ways for agents to make transfers in dynamic TU-games and thereby influence their payoffs. In a first step we presented different ways of introducing transfers into the setting of dynamic TU-games. However, for some particular TU-games different transfer systems seemed in order. We discusses the case of the market game and the class of voting games.

Also other avenues of research present themselves. First, introducing costly transfers and allowing for agents to borrow from a third party could make our model richer. We assume that transfers are not costly, and that agents can only borrow from their own future. However, in several situations, e.g. in the market game, it seems reasonable that transfers are costly. Reshuffling resources among periods (or production places), usually is associated with costs for the firms, may it be due to transportation or storage. Also the influence of an interest rate has to be considered. Moreover, agents can borrow money from a bank or another third party; they can take out a mortgage or run bankrupt. None of these possibilities is considered in our model.

Second, when considering some cooperative problems it might seem reasonable to not only let individuals make transfers, but also give coalitions the possibility to transfer worth among periods. Coalitional transfers are mentioned in Kranich et al. (2005) where several core concepts for dynamic games are discussed and compared. One could implement coalitional transfers into our model by assuming that players simultaneously choose a transfer plan, specifying for each coalition how much of the worth should be transferred and to which period. A transfer is carried out only if all agents in a coalition agree on the transfer. This reflects the idea that coalitions work together and therefore players of a coalition should agree on how to distribute the corresponding worths. Of course several issues might arise from this approach. First, coordination of the agents in a coalition plays then a prominent role. If the agents do not agree, no transfer takes place. Second, discontinuity in utility of the players might arise, as small changes in strategies might lead to a big change in utility. If one models the coalitional transfers as above the players in the non-cooperative game remain the individuals. However, it is also reasonable to assume for coalitions to be the players in the non-cooperative games and then check if strong Nash equilibria exist.

Another extension of our model is to assume that the characteristic function in each stage evolves endogenously. We assume that the sequence of TU-games is externally given, however, the game played tomorrow might crucially depend on the game played today and, in particular, on how the game is played. Incorporating history dependence into our model seems to be a natural next step. One possibility is to introduce transfers into the model of Filar \& Petrosjan (2000). They consider dynamic cooperative games in characteristic function form, where the characteristic function evolves over time. They 
model the development of a future characteristic function via a difference or differential equation that is influenced not only by the current characteristic function but also by the solution concept applied to it.

Another restriction of our model is that we assume that the characteristic function in each stage of the dynamic TU game is common knowledge. Nevertheless uncertainty plays an important role in many surplus sharing problems. Habis \& Herings (2011) introduce so called transferable utility games with uncertainty (TUU-games). In a TUUgame the uncertainty is with regard to payoffs of coalitions. Which game is played is state dependent, and one out of a finite number of states is eventually realized. By taking this into account, we would be able to extend our current work and capture situations in which agents make decisions under uncertainty. In this way non-cooperative games with incomplete information can be modeled and show existence of Bayesian Nash equilibria would be the main task.

\subsection{APPENDIX}

\section{Proof of Theorem 6.3.}

Proof. For $k=2$ fix player $i$ and $\alpha \in \mathcal{S}_{N}^{2}$ such that $\alpha_{j} \in \mathcal{D}_{j}^{2}(\alpha)$ for all $j \in N \backslash\{i\}$. Agent $i$ faces the following maximization problem.

$$
\begin{aligned}
& \max _{\alpha_{i}:\left(\alpha_{i}, \alpha_{-i}\right) \in \mathcal{D}_{i}^{2}(\alpha)} \tilde{u}_{i}(\alpha) \\
& \text { s.t. } \\
& g_{t}(\alpha)=-\alpha_{i}^{t}-\min _{S \in S^{i}}\left\{v^{t}(S)+\sum_{j \in S \backslash\{i\}} \alpha_{j}^{t}\right\} \leq 0 \\
& h(\alpha)=\sum_{t=1}^{T} \alpha_{i}^{t}=0 .
\end{aligned}
$$

Note first, that all inequality constraints are binding, only in the case where $\min _{S \in S^{i}}\left\{v^{t}(S)+\sum_{j \in S \backslash\{i\}} \alpha_{j}^{t}\right\}=0$ for all $t \in\{1, \ldots, T\}$. However, if this is the case, the unique strategy of player $i$ is to make zero transfers. Hence, there is nothing to prove. So, suppose the first $l<T$ inequality constraints are binding. We first show that the non degenerate constraint qualification is satisfied.

$$
\left(\begin{array}{ccc}
\frac{\partial g_{1}}{\partial \alpha_{i}^{\top}}(\alpha) & \ldots & \frac{\partial g_{1}}{\partial \alpha_{i}^{T}}(\alpha) \\
\vdots & \ddots & \vdots \\
\frac{\partial g_{l}}{\partial \alpha_{i}^{1}}(\alpha) & \ldots & \frac{\partial g_{l}}{\partial \alpha_{i}^{T}}(\alpha) \\
\frac{\partial h}{\partial \alpha_{i}^{1}}(\alpha) & \ldots & \frac{\partial h}{\partial \alpha_{i}^{T}}(\alpha)
\end{array}\right)=\left(\begin{array}{cccccc}
-1 & 0 & \ldots & 0 & \ldots & 0 \\
0 & -1 & \ldots & 0 & \ldots & 0 \\
\vdots & & \ddots & \vdots & & \vdots \\
0 & 0 & \ldots & -1 & \ldots & 0 \\
1 & 1 & \ldots & 1 & \ldots & 1
\end{array}\right)
$$


The rank of the Jacobian of the binding constraints is $l+1$ and the non degenerate constraint qualification is satisfied. We aim to maximize the Lagrangian

$$
L\left(\alpha, \lambda_{1}, \ldots, \lambda_{T}, \mu\right)=\tilde{u}_{i}(\alpha)+\sum_{t=1}^{T} \lambda_{t}\left[\alpha_{i}^{t}+\min _{S \in S^{i}}\left\{v^{t}(S)+\sum_{j \in S \backslash\{i\}} \alpha_{j}^{t}\right\}\right]-\mu\left[\sum_{t=1}^{T} \alpha_{i}^{t}\right]
$$

The KKT conditions are:

1. $\frac{\partial L}{\partial \alpha_{i}^{t}}(\alpha)=0$ for $t=1, \ldots, T$

2. $\lambda_{t}\left(\alpha_{i}^{t}+\min _{S \in S^{i}}\left\{v^{t}(S)+\sum_{j \in N} \alpha_{j}^{t}\right\}\right)=0$ for $t=1, \ldots, T$

3. $\lambda_{t} \geq 0$ for $t=1, \ldots, T$

4. $\alpha_{i}^{t}+\min _{S \in S^{i}}\left\{v^{t}(S)+\sum_{j \in S \backslash\{i\}} \alpha_{j}^{t}\right\} \geq 0$ for $t=1, \ldots, T$ and $S \in S^{i}$

5. $\sum_{t=1}^{T} \alpha_{i}^{t}=0$

Observe that the second KKT condition is satisfied if $\lambda_{t}=0$ or $\alpha_{i}^{t}=\min _{S \in S^{i}}\left\{v^{t}(S)+\right.$ $\left.\sum_{j \in S \backslash\{i\}} \alpha_{j}^{t}\right\}$. Suppose $\lambda_{t}=0$ for some $t \in\{1, \ldots, T\}$. Then by the first KKT condition:

$$
\frac{\partial L}{\partial \alpha_{i}^{t}}(\alpha)=\frac{\partial \tilde{u}_{i}}{\partial \alpha_{i}^{t}}(\alpha)+\lambda_{t}-\mu=0
$$

Hence,

$$
\mu=\frac{\partial \tilde{u}_{i}}{\partial \alpha_{i}^{t}}(\alpha)
$$

This implies $\frac{\partial \tilde{u}_{i}}{\partial \alpha_{i}^{t}}(\alpha)=\frac{\partial \tilde{u}_{i}}{\partial \alpha_{i}^{t^{\prime}}}(\alpha)$ for all $t, t^{\prime} \in\{1, \ldots T\}$ with $\lambda_{t}=0$ and $\lambda_{t^{\prime}}=0$. Suppose $-\alpha_{i}^{t^{\prime \prime}}=\min _{S \in S^{i}}\left\{v^{t^{\prime \prime}}(S)+\sum_{j \in S^{i} \backslash\{i\}} \alpha_{j}^{t^{\prime \prime}}\right\}$ for some $t^{\prime \prime} \in\{1, \ldots T\}$. Then by the first KKT condition:

$$
\mu=\frac{\partial \tilde{u}_{i}}{\partial \alpha_{i}^{t^{\prime \prime}}}(\alpha)+\sum_{S \in S^{i}} \lambda_{t^{\prime \prime}, S}
$$

Suppose $\lambda_{t^{\prime \prime}} \geq 0$. Then from 21 and 22 it follows

$$
\frac{\partial \tilde{u}_{i}}{\partial \alpha_{i}^{t}}(\alpha)=\frac{\partial \tilde{u}_{i}}{\partial \alpha_{i}^{t^{\prime \prime}}}(\alpha)+\lambda_{t^{\prime \prime}}
$$


for $t \in\{1, \ldots T\}$ such that $\lambda_{t}=0$. By concavity of $\tilde{u}_{i}$ and the third KKT condition

$$
\frac{\partial \tilde{u}_{i}}{\partial \alpha_{i}^{t}}(\alpha) \geq \frac{\partial \tilde{u}_{i}}{\partial \alpha_{i}^{t^{\prime \prime}}}(\alpha)
$$

Observe that $v_{2, \alpha}^{t}(S)>0$.

For $k=3$ fix $i \in N$ and $\alpha \in \mathcal{S}_{N}^{3}$ such that $\alpha_{j} \in \mathcal{D}_{j}^{3}(\alpha)$ for all $j \in N \backslash\{i\}$. Individual $i$ faces the following maximization problem.

$$
\max _{\alpha_{i}:\left(\alpha_{i}, \alpha_{-i}\right) \in \mathcal{D}_{i}^{3}(\alpha)} \tilde{u}_{i}(\alpha)
$$

s.t.

$g_{t, S}(\alpha)=-\alpha_{i, S}^{t}-\min \left\{c_{S}^{t}(i) ; v^{t}(S)+\sum_{j \in S \backslash\{i\}} \alpha_{j, S}^{t}\right\} \geq 0$ for $t=1, \ldots, T$ and $S \in S^{i}$

$h_{S}(\alpha)=\sum_{t=1}^{T} \alpha_{i, S}^{t}=0$ for all $S \in S^{i}$

Observe that all inequality constraints are binding, only in the case where $\min \left\{c_{S}^{t}(i) ; v^{t}(S)+\sum_{j \in S \backslash\{i\}} \alpha_{j, S}^{t}\right\}=0$ for all $t \in\{1, \ldots, T\}$ and $S \in S^{i}$. However, if this is the case, the unique strategy of player $i$ is to make zero transfers. Hence, there is nothing to prove. Suppose the first $l<T \cdot\left|S^{i}\right|$ inequality constraints are binding.

$$
\left(\begin{array}{ccc}
\frac{\partial g_{1,\{i\}}}{\partial \alpha_{i,\{i\}}^{1}}(\alpha) & \ldots & \frac{\partial g_{1,\{i\}}}{\partial \alpha_{i, N}^{T}}(\alpha) \\
\vdots & \ddots & \vdots \\
\frac{\partial g_{l}}{\partial \alpha_{i,\{i\}}^{1}}(\alpha) & \ldots & \frac{\partial g_{l}}{\partial \alpha_{i, N}^{T}}(\alpha) \\
\frac{\partial h_{\{i\}}^{1}}{\partial \alpha_{i,\{i\}}^{1}}(\alpha) & \ldots & \frac{\partial h_{\{i\}}}{\partial \alpha_{i, N}^{T}}(\alpha) \\
\vdots & \ddots & \vdots \\
\frac{\partial h_{N}}{\partial \alpha_{i,\{i\}}^{1}}(\alpha) & \ldots & \frac{\partial h_{N}}{\partial \alpha_{i, N}^{T}}(\alpha)
\end{array}\right)=\left(\begin{array}{cccccc}
-1 & 0 & \ldots & 0 & \ldots & 0 \\
0 & -1 & \ldots & 0 & \ldots & 0 \\
\vdots & & \ddots & \vdots & & \vdots \\
0 & 0 & \ldots & -1 & \ldots & 0 \\
1 & 1 & \ldots & 0 & \ldots & 0 \\
\vdots & \vdots & \ddots & & & \vdots \\
1 & 1 & \ldots & 1 & \ldots & 1
\end{array}\right)
$$

The rank of the Jacobian of the binding constraints is $l+\left|S^{i}\right|$ and the non degenerate constraint qualification is satisfied. We aim to maximize the Lagrangian

$$
\begin{aligned}
& L\left(\alpha, \lambda_{1,\{i\}}, \ldots, \lambda_{T, N}, \mu_{\{i\}}, \ldots \mu_{N}\right) \\
& =\tilde{u}_{i}(\alpha)+\lambda_{1,\{i\}}\left[\alpha_{i,\{i\}}^{1}+\min \left\{c_{\{i\}}^{1}(i) ; v^{1}(i)\right]+\ldots\right. \\
& +\lambda_{T, N}\left[\alpha_{i, N}^{T}+\min \left\{c_{N}^{T}(i) ; v^{T}(N)+\sum_{j \in N \backslash\{i\}} \alpha_{j, N}^{T}\right]\right. \\
& -\mu_{\{i\}} \sum_{t=1}^{T} \alpha_{i,\{i\}}^{t}-\ldots-\mu_{N} \sum_{t=1}^{T} \alpha_{i, N}^{t}
\end{aligned}
$$


The KKT conditions are:

1. $\frac{\partial L}{\partial \alpha_{i, S}^{t}}(\alpha)=0$ for $t=1, \ldots, T$ and $S \in S^{i}$

2. $\lambda_{t, S}\left(\alpha_{i, S}^{t}+\min \left\{c_{S}^{t}(i) ; v^{t}(S)+\sum_{j \in N \backslash\{i\}} \alpha_{j, S}^{t}\right\}\right)=0$ for $t=1, \ldots, T$ and $S \in S^{i}$

3. $\lambda_{t, S} \geq 0$ for $t=1, \ldots, T$ and $S \in S^{i}$

4. $\alpha_{i, S}^{t}+\min \left\{c_{S}^{t}(i) ; v^{t}(S)+\sum_{j \in S \backslash\{i\}} \alpha_{j, S}^{t}\right\} \geq 0$ for $t=1, \ldots, T$ and $S \in S^{i}$

5. $\sum_{t=1}^{T} \alpha_{i, S}^{t}=0$ for $S \in S^{i}$

Observe that the second KKT condition is satisfied if $\lambda_{t, S}=0$ or $\alpha_{i, S}^{t}=$ $-\min \left\{c_{S}(i)^{t} ; v^{t}(S)+\sum_{j \in S \backslash\{i\}} \alpha_{i, S}^{t}\right\}$. Suppose $\lambda_{t^{\prime}, S^{\prime}}=0$ for some $t^{\prime} \in\{1, \ldots, T\}$ and $S^{\prime} \in S^{i}$. Then by the first KKT condition:

$$
\frac{\partial L}{\partial \alpha_{i, S}^{t^{\prime}}}(\alpha)=\frac{\partial \tilde{u}_{i}}{\partial \alpha_{i, S}^{t^{\prime}}}(\alpha)+\lambda_{t, S}-\mu_{S^{\prime}}=0
$$

Hence,

$$
\mu_{S^{\prime}}=\frac{\partial \tilde{u}_{i}}{\partial \alpha_{i, S^{\prime}}^{t}}(\alpha)
$$

This implies $\frac{\partial \tilde{u}_{i}}{\partial \alpha_{i, S^{\prime}}^{t^{\prime}}}(\alpha)=\frac{\partial \tilde{u}_{i}}{\partial \alpha_{i, S^{\prime}}^{t^{\prime \prime}}}(\alpha)$ for all $t^{\prime}, t^{\prime \prime} \in\{1, \ldots T\}$ with $\lambda_{t^{\prime}, S^{\prime}}=0$ and $\lambda_{t^{\prime \prime}, S^{\prime}}=0$. Suppose $\alpha_{i, S^{\prime}}^{t^{\prime \prime \prime}}=-\min \left\{c_{S^{\prime}}(i)^{t^{\prime \prime \prime}} ; v^{t^{\prime \prime \prime}}\left(S^{\prime}\right)+\sum_{j \in S^{\prime} \backslash\{i\}} \alpha_{i, S^{\prime}}^{t^{\prime \prime \prime}}\right\}$ for some $t^{\prime \prime \prime} \in\{1, \ldots T\}$. Then by the first KKT condition:

$$
\mu_{S^{\prime}}=\frac{\partial \tilde{u}_{i}}{\partial \alpha_{i, S^{\prime}}^{t^{\prime \prime \prime}}}(\alpha)+\lambda_{t^{\prime \prime \prime}, S^{\prime}}
$$

Suppose $\lambda_{t^{\prime \prime \prime}, S^{\prime}} \geq 0$. Then from 23 and 24 it follows

$$
\frac{\partial \tilde{u}_{i}}{\partial \alpha_{i, S^{\prime}}^{t^{\prime}}}(\alpha)=\frac{\partial \tilde{u}_{i}}{\partial \alpha_{i, S^{\prime}}^{t^{\prime \prime \prime}}}(\alpha)+\lambda_{t^{\prime \prime \prime}, S^{\prime}}
$$

for $t^{\prime} \in\{1, \ldots T\}$ such that $\lambda_{t^{\prime}, S^{\prime}}=0$. By concavity of $\tilde{u}_{i}$ and the third KKT condition

$$
\frac{\partial \tilde{u}_{i}}{\partial \alpha_{i, S^{\prime}}^{t^{\prime}}}(\alpha) \geq \frac{\partial \tilde{u}_{i}}{\partial \alpha_{i, S^{\prime}}^{t^{\prime \prime \prime}}}(\alpha)
$$

Observe that $v_{3, \alpha}^{t^{\prime \prime \prime}}\left(S^{\prime}\right)=0$, while $v_{3, \alpha}^{t^{\prime}}\left(S^{\prime}\right)>0$. 


\section{B I B L I O G R A P H Y}

Abdulkadiroğlu, A., \& Sönmez, T. 2013. Matching markets: theory and practice. Pages 3-47 of: Acemoglu, D., Arellano, M., \& Dekel, E. (eds), Advances in Economics and Econometrics: Tenth World Congress Volume I: Economic Theory. Cambridge University Press.

Abeledo, H., \& Rothblum, U. G. 1995. Paths to marriage stability. Discrete Applied Mathematics, 63(1), 1-12.

Bando, K., Kawasaki, R., \& Muto, S. 2016. Two-sided matching with externalities: A survey. Journal of the Operations Research Society of Japan, 59(1), 35-71.

Banerjee, S., Konishi, H., \& Sönmez, T. 2001. Core in a simple coalition formation game. Social Choice and Welfare, 18(1), 135-153.

Banzhaf, J. F. 1965. Weighted voting doesn't work: A mathematical analysis. Rutgers Law Review, 19, 317-343.

Berden, C. 2007. The role of individual intertemporal transfers in dynamic TU-games. Research Memorandum, 07(30).

Black, D. 1958. The theory of committees and elections. Cambridge University Press.

Bloch, F., \& van den Nouweland, A. 2017. Farsighted stability with heterogeneous expectations. Working Paper.

Bogomolnaia, A., \& Jackson, M.O. 2002. The stability of hedonic coalition structures. Games and Economic Behavior, 38(2), 201-230.

Bondareva, O. N. 1963. Some applications of linear programming methods to theory of cooperative games. Problemy Kybernetiki, 10, 119-139.

Brams, S., \& Fishburn, P. 1983. Paradoxes of preferential voting. Mathematics Magazine, 56(4), 207-214.

Brams, S. J. 1994. Theory of Moves. Cambridge University Press.

Brams, S. J., \& Ismail, M. 2018. Stabilizing Cooperative Outcomes in Two-Person Games: Theory and Cases. Working Paper. 
Brandt, F., Geist, C., \& Peters, D. 2017. Optimal bounds for the no-show paradox via SAT solving. Mathematical Social Sciences, 90, 18-27.

Casajus, A., \& Hüttner, F. 2013. Null players, solidarity, and the egalitarian Shapley values. Journal of Mathemenatical Economics, 49(1), 58-61.

Casella, A. 2005. Storable votes. Games and Economic Behavior, 51(2), 391-419.

Chwe, M. S.-Y. 1994. Farsighted Coalitional Stability. Journal of Economic Theory, 63(2), 299-325.

Cook, W., Gerards, A. M. H., Schrijver, A., \& Tardos, É. 1986. Sensitivity theorems and integer linear programming. Mathematical Programming, 34(3), 251-264.

de Condorcet, M. 1785. Essai sur l'application de l'analyse à la probabilité des décisions rendues à la pluralité des voix. Paris: De l'Imprimerie royale.

Deegan, J., \& Packel, E. W. 1978. A new index of power for simple n-person games. International Journal of Game Theory, 7(2), 113-123.

Diamantoudi, E, \& Xue, L. 2003. Farsighted Stability in Hedonic Games. Social Choice and Welfare, 21(1), 39-61.

Dimitrov, D., Kasper, L., \& Yang, Y. 2018. Gender consistent resolving rules in marriage problems. Discrete Applied Mathematics, 247, 147-151.

Drèze, J. H., \& Greenberg, J. 1980. Hedonic coalitions: optimality and stability. Econometrica, 48(4), 987-1003.

Duddy, C. 2014. Condorcet's principle and the strong no show paradoxes. Theory and Decision, 77(2), 275-285.

Dutta, B., \& Vartiainen, H. 2017. Coalition Formation and History Dependence. Working Paper.

Dutta, B, \& Vohra, R. 2017. Rational expectations and farsighted stability. Theoretical Economics, 12(3), 1191-1227.

Felsenthal, D. S., \& Nurmi, H. 2016. Two types of participation failure under nine voting methods in variable electorates. Public Choice, 168, 115-135.

Felsenthal, D. S., \& Tideman, N. 2013. Varieties of failure of monotonicity and participation under five voting methods. Theory and Decision, 75(1), 59-77. 
Filar, J. A., \& Petrosjan, L. A. 2000. Dynamic cooperative games. International Game Theory Review, 2(1), 47-65.

Fishburn, P. C., \& Brams, S. J. 1983. Paradoxes of preferential voting. Mathematics Magazine, 56(4), 207-214.

Gale, D., \& Shapley, L. S. 1962. College admissions and the stability of marriage. The American Mathematical Monthly, 69(1), 9-15.

Gillies, D. 1959. Solutions to General Non-zero Games. Pages 47-85 of: Kuhn, A. W., \& Luce, R. D. (eds), Contributions to the Theory of Games. Princeton University Press.

Habis, H., \& Herings, J.-J. P. 2010. A note on the weak sequential core of dynamic TU games. International Game Theory Review, 12(4), 407-416.

Habis, H., \& Herings, J.-J. P. 2011. Transferable utility games with uncertainty. Journal of Economic Theory, 146(5), 2126-2139.

Harsanyi, J. C. 1974. An Equilibrium-point Interpretation of Stable Sets and a Proposed Alternative Definition. Management Science, 20(11), 1472-1495.

Herings, P. J.-J., Mauleon, A., \& Vannetelbosch, V. J. 2009. Farsightedly Stable Networks. Games and Economic Behavior, 67, 526-541.

Jimeno, J. L., Pérez, J., \& García, E. 2009. An extension of the Moulin no show paradox for voting correspondences. Social Choice and Welfare, 33(3), 343-359.

Joosten, R. 1996. Dynamics, equilibria and values. Ph.D. thesis, Maastricht University.

Jordan, J. S. 2006. Pillage and Property. Journal of Economic Theory, 131(1), 26-44.

Ju, Y., Borm, P., \& Ruys, P. 2007. The consensus value: a new solution concept for cooperative games. Social Choice and Welfare, 28(4), 685-703.

Kakutani, S. 1941. A generalization of Brouwer's fixed point theorem. Duke Mathematical Journal, 8(3), 457-459.

Kimya, M. 2018. Equilibrium Coalitional Behavior. Working Paper.

Klaus, B., \& Klijn, F. 2005. Stable matchings and preferences of couples. Journal of Economic Theory, 121(1), 75-106.

Klijn, F., \& Massó, J. 2003. Weak stability and a bargaining set for the marriage model. Games and Economic Behavior, 42(1), 91-100. 
Knuth, D. 1976. Marriages Stables. Les Presses de l’Université Montréal.

Kojima, F. 2015. Recent developments in matching theory and its practical applications. Working Paper.

Kojima, F., \& Manea, M. 2010. Axioms for deferred acceptance. Econometrica, 78(2), 633-653.

Kojima, F., \& Ünver, M. U. 2014. The "Boston" school-choice mechanism: an axiomatic approach. Economic Theory, 55(3), 515-544.

Kramer, G. H. 1977. A dynamical model of political equilibrium. Journal of Economic Theory, 16(2), 310-334.

Kranich, L., Perea, A., \& Peters, H. 2005. Core concepts for dynamic TU games. International Game Theory Review, 7(1), 43-61.

Lehrer, E., \& Scarsini, M. 2013. On the core of dynamic cooperative games. Dynamic Games and Applications, 3(3), 359-373.

Ma, J. 1996. On randomized matching mechanisms. Economic Theory, 8(2), 377-381.

Mauleon, A., \& Vannetelbosch, V. J. 2004. Farsightedness and cautiousness in coalition formation games with positive spillovers. Theory and Decision, 56(3), 291-324.

Mauleon, A., Vannetelbosch, V. J., \& Vergote, W. 2011. Von Neumann-Morgenstern farsightedly stable sets in two-sided matching. Theoretical Economics, 6(3), 499-521.

McVitie, D. G., \& Wilson, L. B. 1970. Stable marriage assignment for unequal sets. BIT Numerical Mathematics, 10(3), 295-309.

Morales, D. R., \& Vermeulen, D. 2009. Existence of equilibria in a decentralized two-level supply chain. European Journal of Operational Research, 197(2), 642-658.

Morrill, T. 2013. An alternative characterization of the deferred acceptance algorithm. International Journal of Game Theory, 42(1), 19-28.

Moulin, H. 1988. Condorcet's principle implies the no show paradox. Journal of Economic Theory, 45(1), 53-64.

Myerson, R. B. 1991. Game Theory: analysis of conflict. Harvard University Press.

Núñez, M., \& Sanver, M. R. 2017. Revisiting the connection between the no-show paradox and monotonicity. Mathematical Social Sciences, 90, 9-17. 
Nash, J. F. 1950a. The bargaining problem. Econometrica, 18(2), 155-162.

Nash, J. F. 1950b. Equilibrium points in n-person games. Proceedings of the National Academy of Sciences of the United States of America, 36(1), 48-49.

Nash, J. F. 1951. Non-cooperative games. Annals of Mathematics, 54(2), 286-295.

Nurmi, H. 2002. Voting procedures under uncertainty. Springer Verlag.

Pérez, J. 2001. The strong no show paradoxes are a common flaw in Condorcet voting correspondences. Social Choice and Welfare, 18(3), 601-616.

Predtetchinski, A. 2007. The strong sequential core for stationary cooperative games. Games and Economic Behavior, 61(1), 50-66.

Ray, D. 2007. A Game-Theoretic Perspective on Coalition Formation. Oxford University Press.

Ray, D., \& Vohra, R. 2015. The Farsighted Stable Set. Econometrica, 83(3), 977-1011.

Ray, D., \& Vohra, R. 2017. Maximality in the Farsighted Stable Set. Working Paper.

Rosenthal, E. C. 1990a. Monotonicity of solutions in certain dynamic cooperative games Economics Letters, 34(3), 221-226.

Rosenthal, E. C. 1990b. Monotonicity of the core and value in dynamic cooperative games. International Journal of Game Theory, 19(1), 45-57.

Roth, A. E. 1984. The evolution of the labor market for medical interns and residents: a case study in game theory. Journal of Political Economy, 92(6), 991-1016.

Roth, A. E., \& Sotomayor, M. A. O. 1990. Two-Sided Matching: A Study in GameTheoretic Modeling and Analysis. Econometric Society Monographs. Cambridge University Press.

Roth, A. E., \& Vande Vate, J. H. 1990. Random paths to stability in two-sided matching. Econometrica, 58(6), 1475-1480.

Sasaki, H., \& Toda, M. 1996. Two-sided matching problems with externalities. Journal of Economic Theory, 70(1), 93-108.

Schmeidler, D. 1969. The nucleolus of a characteristic function game. SIAM Journal on Applied Mathematics, 17(6), 1163-1170.

Shapley, L. S. 1953a. Additive and non-additive set functions. Ph.D. thesis, Department of Mathematics, Princeton University. 
Shapley, L. S. 1953b. A value for n-person games. Pages 307-317 of: Kuhn, H. W., \& Tucker, A. W. (eds), Contributions to the theory of games II. Princeton: Princeton University Press.

Shapley, L. S. 1967. On balanced sets and cores. Naval Research Logistics Quarterly, 14, 453-560.

Shapley, L. S., \& Shubik, M. 1954. A method for evaluating the distribution of power in a committee system. American Political Science Review, 48(3), 787-792.

Shapley, L. S., \& Shubik, M. 1969. On market games. Journal of Economic Theory, 1(1), 9-25.

Simpson, P. B. 1969. On defining areas of voter choice: Professor Tullock on stable voting. The Quarterly Journal of Economics, 83(3), 478-490.

Sobolev, A. I. 1975. The characterization of optimality principles in cooperative games by functional equations. Mathematical methods in the social sciences, 6, 94-151.

Tijs, S. H. 1981. Bounds for the core of a game and the t-value. Tech. rept. Tilburg University, School of Economics and Management.

Tijs, S. H. 1987. An axiomatization of the $\tau$-value. Mathematical Social Sciences, 13, $177-181$.

Tijs, S. H., \& Lipperts, F. A. S. 1982. The Hypercube and the core cover of n-person cooperative games. Cahiers du Centre d'Études de Recharche Opérationelle, 24(3), 27-37.

van Deemen, A., \& Vergunst, N. P. 1998. Empirical evidence of paradoxes of voting in Dutch elections. Public Choice, 97(3), 475-490.

van den Brink, R., Funaki, Y., \& Ju, Y. 2013. Reconciling marginalism with egalitarianism: consistency, monotonicity, and implementation of egalitarian Shapley values. Social Choice and Welfare, 40(3), 693-714.

Vartiainen, H. 2018. History Dependent Stable Set as a Solution to Abstract Games. Working Paper.

von Neumann, J., \& Morgenstern, O. 1944. Theory of Games and Economic Behaviour. Princeton University Press.

Xue, L. 1998. Coalitional Stability under Perfect Foresight. Economic Theory, 11(3), 603-627. 
Young, H. P. 1977. Extending Condorcet's rule. Journal of Economic Theory, 16(2), $335-353$.

Zhou, L. 1994. A new bargaining set of a N-person game and endogenous coalition formation. Games and Economic Behaviour, 6(3), 512-526. 

In our daily life, we are often confronted with problems of matching, voting and cooperation: students have to be matched to schools, and organ transplants need to be matched to patients; presidents are elected, and laws are voted on; and at work projects are undertaken in groups. This thesis covers different topics in matching and voting theory, as well as cooperative game theory. The first chapter discusses two-sided matching problems in which workers' employment experience shapes firms' preferences. Assuming that experience assigns to each worker a set of firms he has been employed by, we first show that there are experience configurations for which no stable matching exists. We then present a sufficient condition guaranteeing existence of stable matchings for any configuration. The condition stipulates the idea that firms are size-sensitive with respect to the experience of each worker and it allows us characterize the set of stable matchings. We also provide an example showing that experience size-sensitivity does not assure the existence of a firm optimal stable matching. We contribute with the third chapter to the branch of axiomatic characterization within the field of matching theory. We assume that a resolving rule guides the selection of blocking pairs and show that two axioms (independence and top optimality) transform such a rule into a gender consistent one. Stable matchings can be reached when starting from an arbitrary individually rational matching and iteratively satisfying the pair selected by a gender consistent resolving rule.

The third chapter focuses on voting theory. We consider voting correspondences that are, besides Condorcet Consistent, immune against the two strong no show paradoxes. That is, it cannot happen that if an additional voter ranks a winning alternative on top then that alternative becomes losing, and that if an additional voter ranks a losing alternative at bottom then that alternative becomes winning. We identify the maximal voting correspondence that satisfies Condorcet Consistency and is immune against the two strong no show paradoxes. In particular, voting functions (single-valued voting correspondences) that are Condorcet Consistent and immune against the two paradoxes must select from this maximal correspondence, and we demonstrate several ways in which this can or cannot be done. We also consider a weaker version of Condorcet Consistency.

The last two chapters discuss topics of cooperation. In the fourth chapter, we introduce a new concept of farsightedness and base a new solution concept for abstract games on it. An abstract game consists of a set of states and specifies what coalitions are allowed to replace one state by another one. Agents are called farsighted if they compare the status quo to the long-term outcome following their deviation rather than to the status 
they actually deviate to. What the literature has ignored so far is that if a coalition does not move out of the status quo, they might still expect another coalition to do so. Specifically, above definition of farsightedness implies that agents ignore this possibility in their reasoning. So, in fact, agents are not fully farsighted. Expectation functions assign to each state a (potentially) different state and a coalition that moves from the former to the latter, thereby creating paths between states. This endows agents with an expectation about what any potential deviation entails—namely the path of the prescribed further moves. We extend these functions by capturing coalitions' expectations about the consequences of not moving out of a state. We impose three stability and optimality axioms on extended expectation functions that reflect full farsightedness and rationality. We then show that an extended expectation function satisfies our axioms if and only if it can be associated with a non-cooperative equilibrium of the abstract game. We finally apply our solution to games in characteristic function form and matching problems.

The fifth chapter analyzes individuals' behavior if agents have to repeatedly cooperate with others. Although cooperative game theory normally abstracts away of any strategic interaction between players, we argue that in a repeated set up players would act strategically if they could. We intend to incorporate and predict such (strategic) behavior by modeling non-cooperative games based on a dynamic game with transferable utility and point-wise solution concepts for them. We model the strategic interaction between agents by allowing them to transfer some worth among periods, and, hence, indirectly affect their payoffs and the payoffs of the other players. We discuss different transfer systems, specifying what worth agents can transfer and identify Nash equilibria in the resulting non-cooperative games. Furthermore, we characterize the Nash equilibria and discuss uniqueness. We also consider particular classes of TU-games, such as market games and voting games and introduce transfer systems that seem to be suited best for these games: in market games, agents can transfer their initial endowment among periods, and in the context of voting games, we allow agents to shift their voting weights over time. Based on this idea we derive non-cooperative games and further discuss existence and uniqueness of Nash equilibria. 
This annex will cover the valorisation of my doctoral thesis. I will provide answers to a number of questions that will guide this addendum on valorisation. I will elaborate on the social and/or economic relevance of the present research, the target group of the present research beyond academia, what activities or products can be created from the present research, and how innovative this research is. The last question asks for a plan to implement valorisation. I will then discuss the valorisation itself.

\section{What is the social/ economic relevance of your research?}

This thesis contributes to topics in matching theory, social choice theory and cooperative game theory.

Matching theory: The question on how to pair agents/ objects from different sides of the market, has never lost its relevance. How can we match workers to firms; students to schools; and kidney donors to patients? There have been made great contributions to society, in particular in school choice and kidney exchange problems. There are two Nobel Prize winners honored for their contributions to matching theory and mechanism design: Alvin Roth and Lloyd Shapley. Clearly, just because we already solved some real-life problems, by far not all our questions have been answered. What influence does a more complicated preference structure have on known results? If we would like to use a mechanism to allocate objects to agents or agents to agents, respectively, which one should we use? Can we make a decision based on some desired properties? And if so, what properties should that be? We discuss some of the above questions in this thesis.

Voting theory: Voting theory applies in any situation where we vote, may it be on political parties, or candidates, or simply on activities that we want to undertake as a group. So, we are all affected by the results presented in this field of research. The question on how to aggregate voters' ballots is not new, but it will also never lose its relevance. We all think that we should use our right to vote and that by voting we can only benefit. However, as it turns out, that is not true. This fact is discussed in this thesis.

Cooperation: The topic of cooperation is important to society. We are confronted with cooperation on a dailz basis, may it be cooperation on individual level, as in our jobs, or on country level, e.g. European Union. Sometimes, cooperation takes place repeatedly over time, sometimes just once. In any case, also the topic of 
cooperation is never going to lose its relevance. Repeated cooperation is discussed in this thesis.

2. To whom, in addition to the academic community, are your research results of interest and why?

As mentioned in the previous paragraph, some of the questions answered in this thesis are not of purely academic interest. Instead, these are questions raised by politicians and policy makers. Moreover, each individual might be affected by the results presented in this thesis. In particular, this thesis might raise new questions, to which people might wish to find answers.

3. Into which concrete products, services, processes, activities or commercial activities will your results be translated and shaped?

I agree that, in general, research can be translated in products, services, processes and (commercial) activities. However, many of them are not applicable to this thesis. I can imagine that some of the research presented in this thesis can be transformed into non-academic publications or used as teaching materials. Students that take a course in economic theory/game theory could read them in class in order to learn how to evaluate academic papers. I will elaborate on this further in section 5 of this annex.

4. To what degree can your results be called innovative with respect to the existing range of products, services, processes, activities, and commercial activities?

My research does not have any relationship to existing products, services, processes, activities, or commercial activities. The innovation(s) presented in this thesis are conceptual and analytical contributions to the research in above fields.

5. How will this/these plan(s) for valorisation be shaped? What is the schedule, are there risks involved, what market opportunities are there and what are the costs involved?

I already argued that the range of products into which this thesis can be translated is somehow limited. I suggested that some of this research can be published (in a modified version) in non-academic journals which would make the implementation cost efficient. Moreover, this thesis, or the corresponding papers could be used as teaching material, which would be cost efficient as well. A disadvantage of this research is, at least as this valorisation suggests, that there are no market opportunities for products created on its basis. 
I would like to spend a few sentences on evaluating valorisation. I think that the valorisation, in particular, the above questions give the impression that research is only valuable, if it is implementable or it can be transformed in product, services, processes, and (commercial) activities. I do not share this opinion. What is the value of research? To me, the value of research is that it creates knowledge. Moreover, it creates new questions. Answers to these questions generate knowledge again. Sometimes we do not immediately see the impact of research to society, which does not mean, that there is no value of the research to society. 

Laura Kasper was born in Saabrücken, Germany on March 30, 1989. In 2008 she received her Gymnasium diploma from Marienschule Saarbrücken. In the same year, she started studying Business Administration at Saarland University. During her studies in Business Administration, she discovered that she has a particular interest for economics. In October 2013 she started a Master in Economics, Finance and Philosophy at Saarland University while working as a Research and Teaching Assistant at the Chair of Economic Theory, Saarland University. She received her Master's degree with distinction in February 2015. Shortly, after that, in April 2016, she started a PhD at the Quantitative Economics Department of Maastricht Unversity under the supervision of Hans Peters and Dries Vermeulen. The results of her $\mathrm{PhD}$ research are presented in this thesis. She presented her papers at various international conferences, and one of them is published in an international peer-reviewed journal, Discrete Applied Mathematics. Up to today, she holds a position as Research and Teaching Assistant at the Chair of Economic Theory at Saarland University. 
